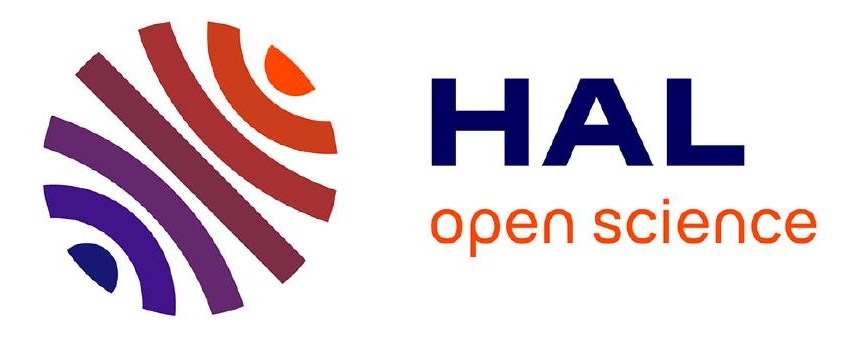

\title{
Social touch promotes interfemale communication via activation of parvocellular oxytocin neurons
}

\author{
Yan Tang, Diego Benusiglio, Arthur Lefevre, Louis Hilfiger, Ferdinand \\ Althammer, Anna Bludau, Daisuke Hagiwara, Angel Baudon, Pascal Darbon, \\ Jonas Schimmer, et al.
}

\section{To cite this version:}

Yan Tang, Diego Benusiglio, Arthur Lefevre, Louis Hilfiger, Ferdinand Althammer, et al.. Social touch promotes interfemale communication via activation of parvocellular oxytocin neurons. Nature Neuroscience, 2020, 23 (9), pp.1125-1137. 10.1038/s41593-020-0674-y · hal-02988845

\section{HAL Id: hal-02988845 \\ https://hal.science/hal-02988845}

Submitted on 4 Nov 2020

HAL is a multi-disciplinary open access archive for the deposit and dissemination of scientific research documents, whether they are published or not. The documents may come from teaching and research institutions in France or abroad, or from public or private research centers.
L'archive ouverte pluridisciplinaire HAL, est destinée au dépôt et à la diffusion de documents scientifiques de niveau recherche, publiés ou non, émanant des établissements d'enseignement et de recherche français ou étrangers, des laboratoires publics ou privés. 
Yan Tang $^{1 *}$, Diego Benusiglio ${ }^{1 *}$, Arthur Lefevre ${ }^{1,2} *$, Louis Hilfiger $^{2 *}$, Ferdinand Althammer ${ }^{1,3}$, Anna Bludau ${ }^{4}$, Daisuke Hagiwara ${ }^{1}$, Angel Baudon ${ }^{2}$, Pascal Darbon ${ }^{2}$, Jonas Schimmer ${ }^{1}$, Matthew K. Kirchner $^{3}$, Ranjan K. Roy ${ }^{3}$, Shiyi Wang ${ }^{1}$, Marina Eliava ${ }^{1}$, Shlomo Wagner ${ }^{5}$, Martina Oberhuber ${ }^{6}$, Karl K. Conzelmann ${ }^{6}$, Martin Schwarz ${ }^{7}$, Javier E. Stern ${ }^{3}$, Gareth Leng ${ }^{8}$, Inga D. Neumann ${ }^{4}$, Alexandre Charlet ${ }^{2 @ \#}$ and Valery Grinevich ${ }^{1,3 @ \#}$

${ }^{1}$ Department of Neuropeptide Research in Psychiatry, Central Institute of Mental Health, Medical Faculty Mannheim, University of Heidelberg, 68159 Mannheim, Germany

${ }^{2}$ Centre National de la Recherche Scientifique (CNRS) and University of Strasbourg, UPR3212, Institute of Cellular and Integrative Neurosciences, 67084 Strasbourg, France

${ }^{3}$ Center for Neuroinflammation and Cardiometabolic Diseases, Georgia State University, Atlanta GA 30303, USA

${ }^{4}$ Department of Neurobiology and Animal Physiology, University of Regensburg, 93040 Regensburg, Germany

${ }^{5}$ Sagol Department of Neurobiology, University of Haifa, Mt. Carmel, Haifa 31905, Israel

${ }^{6}$ Max von Pettenkofer-Institute Virology, Faculty of Medicine and Gene Center, Ludwig Maximilians University 81377 Munich, Germany.

${ }^{7}$ Institute for Experimental Epileptology and Cognition Research, University of Bonn Medical Center, D-53127 Bonn, Germany

${ }^{8}$ Centre for Discovery Brain Sciences, University of Edinburgh, Edinburgh EH8 9XD, UK

${ }^{\$}$ Present address: Department of Biomedical Sciences, Faculty of Biology and Medicine, University of Lausanne, 1005 Lausanne, Switzerland

*Equal first authors

${ }^{\circledR}$ Equal senior authors

${ }^{\#}$ Corresponding authors:

Alexandre Charlet, $\mathrm{PhD}$

Institute of Cellular and Integrative Neurosciences,

INCI CNRS UPR3212

8, Allée du Général Rouvillois

67000 Strasbourg, France

Phone: (33) 607082506

E-mail: acharlet@unistra.fr

Valery Grinevich, MD, $\mathrm{PhD}$

Department of Neuropeptide Research in Psychiatry

Central Institute of Mental Health

Medical Faculty Mannheim

University of Heidelberg

J5, Mannheim, 68159

Germany

Phone: (49) 62117032995

E-mail: valery.grinevich@zi-mannheim.de 
Abstract

51 Oxytocin is a great facilitator of social life, but although its effects on socially-relevant brain regions have been extensively studied, oxytocin neuron activity during actual social interactions remains unexplored. The majority of oxytocin neurons are magnocellular neurons, which

54 simultaneously project to the pituitary and forebrain regions involved in social behaviors. Here, we 55 show that a much smaller population of oxytocin neurons, parvocellular neurons that do not project to the pituitary but which synapse onto magnocellular neurons, is preferentially activated by 57 somatosensory stimuli. This activation is transmitted to the larger population of magnocellular 58 neurons, which consequently show coordinated increases in their activity during social interactions between virgin female rats. Selectively activating these parvocellular neurons promotes social

60 motivation, whereas inhibiting them reduces social interactions. Thus, parvocellular oxytocin 61 neurons, receive somatosensory inputs to control social behavior by coordinating the responses of 62 the much larger population of magnocellular oxytocin neurons. 


\section{INTRODUCTION}

The hypothalamic neuropeptide oxytocin (OT) promotes various types of social behavior ${ }^{1-3}$. OT is mainly synthesized in neurons of the paraventricular (PVN) and supraoptic (SON) nuclei of the hypothalamus. The vast majority of these neurons project to the posterior pituitary, where OT is secreted into the blood for essential physiological effects, such as suckling-induced milk let-down and for regulating uterine contractions during birth ${ }^{4}$. In parallel, these neurons project axonal collaterals to forebrain regions ${ }^{5}$ that express OT receptors (OTRs), including the central nucleus of the amygdala, nucleus accumbens, lateral septum, hippocampus, and medial prefrontal cortex ${ }^{6,7}$. Studies employing microdialysis to measure OT concentrations within socially-relevant brain regions revealed that OT is released in the bed nucleus of stria terminalis, lateral septum, and central nucleus of amygdala during social investigation of a conspecific ${ }^{2,8,9}$. However, to date, no direct measurement of OT neuron activity during actual social interaction of freely moving conspecifics has been performed, although it was recently reported that social approach triggers calcium release in PVN OT neurons in immobilized, head-fixed male mice ${ }^{10}$.

Several studies suggest that female-female interactions are predominantly mediated via somatosensory inputs ${ }^{11,12}$, while other interactions such as male-male, male-female or parental contact may rely on other sensory modalities. However, whether those sensory stimulations can activate OT neurons is unknown since, to this date, there has been no direct recording of activity from identified OT neurons during actual social behavior. In an attempt to address these points, in the current study, we performed ex vivo and in vivo manipulation of OT neurons activity primarily in the PVN - the main source of OT in the brain $^{5}$ - to decipher their involvement in the modulation of social interaction in freely-moving female rats.

\section{RESULTS}

\section{PVN OT neurons are activated upon social interaction}


To identify OT neurons electrophysiologically, we injected a recombinant adeno-associated virus (rAAV-OTp-ChR2-mCherry) bilaterally into the PVN to induce expression of the light-sensitive ion channel Channelrhodopsin-2 (ChR2) under the control of the OT promoter ${ }^{5,13}$. This resulted in 90.4\% of ChR2-expressing neurons being OT-positive, showing the high specificity of the infection in the PVN (Extended Data Fig. 1a). We then recorded individual neurons in the PVN using implanted tetrodes combined with an optic fiber to identify the OT neurons by their electrophysiological response to blue-laser pulses, similar to methods in ${ }^{14}$.

In total, we recorded 90 neurons in 10 adult female rats at the diestrus phase of the ovarian cycle, while monitoring the behavior of the rats and their ultrasonic vocalizations during both open field (OF) exploration and free social interactions (FSI) (Fig. 1a-b). Fifteen of these neurons (in 5 animals) were stringently identified as single OT neurons (Extended Data Fig. 1e). In the open field arena, the patterns of spiking activity of these neurons (Fig. 1d, Extended Data Fig. 2d) were indistinguishable from those of OT neurons observed under basal conditions in anesthetized rats, as these neurons displayed typical OT neurons characteristics ${ }^{15}$. Specifically, they all display low rate of tonic firing $(\sim 1 \mathrm{~Hz})$ with a low index of dispersion of spikes $(<1)$ and a distribution of interspike intervals consistent with random spike generation subject to a prolonged relative refractory period. In contrast, during episodes of FSI with an unfamiliar conspecific, the same neurons fired at a higher rate (mean increase $1.5 \pm 0.4$ spikes/s, $p=0.001, \mathrm{n}=15$; Fig. 1c-d) and more irregularly; the second-by-second firing rates showed a high index of dispersion, reflecting the prominent occurrence of clusters of spikes (Fig. 1d, Extended Data Fig. 1n).

As revealed by cross-correlation analysis, OT neurons also displayed increased synchronicity during FSI (mean pairwise correlation: open field, $0.10 \pm 0.04$; FSI $0.40 \pm 0.08, \mathrm{p}=0.001$, Extended Data Fig. 1k-l). In anesthetized rats, adjacent OT neurons showed no such crosscorrelated activity. We also recorded local field potentials in the PVN and found a significant 
114 increase of oscillatory power in the theta $(5-10 \mathrm{~Hz})$ frequency band during FSI (Extended Data

115 Fig. 1f-h). The spike activity of OT neurons tended to be phase-locked with theta oscillations

116 during FSI, but not in the open field arena (Extended Data Fig. 1i-j). In contrast to OT neurons,

117 non-OT PVN neurons did not show an increase in spiking activity when comparing exploratory

118 behavior and social interaction (Extended Data Fig. 2e-g).

119 Thus, during free social interaction with actual physical contact, OT neurons in the PVN were more

120 active and exhibited frequent clusters of spikes, and this activity was correlated amongst the OT

121 neurons.

\section{Social physical contact increases PVN OT neuron activity}

123 To examine which component of social interaction activates these neurons, we first recorded their

124 neuronal activity during a chambered social interaction (CSI) ${ }^{16}$. In this setup, experimental and

125 stimulus rats were separated by a transparent wall with small holes $(7.5 \mathrm{~mm})$ in it, allowing rats to

126 see, sniff and hear each other, but not to touch each other (CSI, Fig. 1e).

127 OT neurons showed little change in spiking activity between CSI and baseline recordings in OF

128 (CSI: $1.4 \pm 0.4$ spikes/s; open field: $1.0 \pm 0.2$ spikes/s, $p=0.14$; Fig. 1f). When the wall was

129 removed to allow free social interaction (FSI), the same OT neurons displayed a significant increase

130 in activity (FSI: $3.0 \pm 0.4$ spikes/s, p $<0.001$; Fig. 1f), accompanied by an increase in index of

131 dispersion (FSI, $3.2 \pm 0.4$; CSI, $1.3 \pm 0.3, p=0.006$ vs FSI; open-field, $0.9 \pm 0.2, p=0.004$ vs FSI).

132 To estimate the amount of oxytocin axonal release due to the increase in firing rate together with

133 the altered firing pattern, we employed an activity (spike) dependent model of oxytocin secretion ${ }^{17}$

134 (Extended Data Fig. $2 \mathbf{h}$-j) that quantitatively captures the features of stimulus-secretion coupling

135 at the nerve terminals.

136 To dissect which sensory modalities activate OT neurons during FSI, we categorized rat social 137 behaviors into "sniffing", "head-to-head" and "crawled on top" or "being crawled" events and 
constructed peristimulus time histograms (PSTH) of spiking activity before, during, and after the onset of each sequence (Fig. 1g-h). "Crawled on top" and "being crawled" induced the greatest increases in firing rates $(p=0.036, p=0.024$, respectively; Supplementary Video 1), while "sniffing”, “chasing”, and "head-to-head” events induced lesser, non-significant changes (Fig. 1h;

Extended Data Fig. 2a-c). In addition, ultrasonic vocalizations during FSI revealed the appearance of bands between 40 and $90 \mathrm{kHz}$ known to be related to social communication in rats ${ }^{18}$ (Extended Data Fig. 3a-b), but we found no time-locked (in ranges up to $\pm 5 \mathrm{~s}$ ) correlation between OT neuron activity and ultrasonic vocalizations (Extended Data Fig. 3c-e). Although we were not able to discriminate USV individual calls between the two conspecifics, we hypothesized that OT neurons were activated mainly by physical contacts and investigated this further by modeling gentle, non-nociceptive mechanical stimuli.

\section{Gentle non-nociceptive mechanical stimuli trigger OT neuron activation}

To test whether somatosensory stimulation itself is sufficient to increase OT cell activity, we performed controlled tactile stimulations using compressed air delivery ("airpuffs") in isoflurane anaesthetized rats as $\operatorname{in}^{19}$ (Fig. 2a). Stimulation of the skin on the dorsal body region by airpuffs (at three sites) reproducibly activated 19 out of $23(83 \%)$ recorded PVN OT neurons (mean increase $1.3 \pm 0.5$ spikes/s, mean *p = 0.021, Fig. 2a-b and Extended Data Fig. 4a-b).

Airpuffs applied to the abdominal skin produced little or no changes in their activity (mean change $0.5 \pm 0.3$ spikes/s., $p=0.33$ ), and there were no detected effects after stimulation of the anogenital area or the whiskers pad (Extended Data Fig. 4c). For a potential involvement of the olfactory system in PVN OT neurons activation during social interaction, we exposed female rats to either a neutral odor (clean bedding) or to a socially relevant odor (urinated-on female beddings). We found that the exposure to odorants did not elicit significant changes in firing rate or in spike distribution ( $p=0.34, p=0.48$ respectively, Extended Data Fig. 4d-f) in any of the recorded OT neurons. 
162 There was also no difference upon presentation of neutral odor. Hence, we concluded that

163 somatosensory inputs are the dominant signals that activate PVN OT neurons during social 164 interactions.

ParvOT neurons respond to gentle non-nociceptive mechanical stimuli

167 Although the overwhelming majority of OT neurons in the PVN (97\%) are magnocellular 168 (magnOT) neurons, there is also a population of parvocellular OT (parvOT) neurons $(\sim 3 \%)$ that do 169 not project to the pituitary ${ }^{20}$, but which are crucial for the transmission of nociceptive signals to the 170 magnOT cells ${ }^{13}$.

172 To study whether parvOT neurons are also activated by non-nociceptive stimuli, applying airpuffs 173 to conscious rats trained and adapted for short-term immobilization. For this purpose, we first used 174 rats that had been injected systemically with the retrograde tracer Fluorogold ${ }^{\mathrm{TM}}$ to label all neurons 175 in the brain that project outside the blood-brain barrier, including in particular magnOT, but not 176 parvOT neurons. To identify neurons strongly activated by airpuffs, we used the expression of $c$-fos 177 as an indicator of activated OT neurons. Previous studies have found that $c$-fos expression is 178 activated in non-identified OT neuron cell type following social interaction in voles ${ }^{21}$, mice ${ }^{22}$ and

179 rats $^{23}$. Immunocytochemistry revealed the presence of $c$-fos in $30 \%$ of parvOT neurons in the PVN 180 of stimulated rats (average $12.4 \pm 3$ neurons per PVN/hemisphere, n $=4$, Fig. 2c, Supplementary

181 Table 1a), but not in magnOT neurons or in any OT neurons in non-stimulated control rats, 182 indicating that airpuffs specifically applied to the dorsal body region seem to predominantly 183 activate parvOT neurons. In a second step, we labeled parvOT neurons retrogradely by injecting the 184 canine adenovirus serotype 2 (CAV2-Cre $)^{24}$ into the SON, and concomitantly injected the Cre185 responder rAAV expressing mCherry under the control of the OT promoter into the PVN. In line 186 with our previous results, airpuffs induced $c$-fos expression exclusively in retrogradely-labeled 
mCherry-positve OT neurons (average 47.6\%, $7.5 \pm 3$ neurons per PVN/hemisphere, $\mathrm{n}=4$, Fig. 2d,

\section{Supplementary Table 1b).}

To explore the role of parvOT neurons in social interaction and their response to gentle nonnociceptive mechanical stimuli (airpuffs), we chose to manipulate their activity via virallyexpressed DREADDs. To this end, we used a similar Cre-dependent viral-based strategy employing OTp-DIO-hM4D(Gi)-mCherry and OTp-DIO-hM3D(Gq)-mCherry rAAVs (Fig. 2e-f). As a first step, we verified the efficiency of DREADDs in modulating of parvOT neuron activity ex vivo, showing that $\mathrm{hM} 3 \mathrm{D}(\mathrm{Gq})$-CNO-induced parvOT activation significantly increased the spontaneous AP frequency (baseline $0.85 \pm 0.39 \mathrm{~Hz}$ vs CNO $1.31 \pm 0.51 \mathrm{~Hz}, \mathrm{n}=9 ; \mathrm{p}=0.0039$;

Extended Data Fig. 5a-c) and the number of evoked APs (16.18 \pm 3.89 AP vs CNO $22.55 \pm 5.66$

198 AP, $n=11 ; p=0.0314$; Extended Data Fig. 5d-f). Consistent with this, hM4D(Gi)-CNO-induced

199 inhibition $(10 \mu \mathrm{M}, 6 \mathrm{~min})$ significantly decreased both the spontaneous AP frequency (baseline 1.38 $200 \pm 0.38 \mathrm{~Hz}$ vs CNO $0.36 \pm 0.18 \mathrm{~Hz}, \mathrm{n}=7$; $\mathrm{p}=0.0469$; Extended Data Fig. 5g-i) and the number of 201 evoked action potentials (APs) (baseline $13 \pm 2.02$ AP vs CNO $7.75 \pm 2.03 \mathrm{AP}, \mathrm{n}=11 ; \mathrm{p}=0.0007$

\section{Extended Data Fig. 5j-l).}

Following the ex vivo results, we next performed in vivo recording in anaesthetized animals to better understand the airpuff-induced activation of parvOT. For this purpose, PVN parvOT activity was imaged using the GCaMP6s reporter using fiber photometry ${ }^{25}$ (Fig. 2e-h). Then, rats were injected with the DREADD ligand $\mathrm{CNO}\left(3 \mathrm{mg} / \mathrm{kg}\right.$ i.p.) and OT neurons $\mathrm{Ca}^{2+}$ transients were analyzed. Chemogenetic activation of the parvOT neurons enhanced the $\mathrm{Ca}^{2+}$ response to airpuffs $(45 \pm 9 \%$ increase of area under the curve (AUC), $p=0.03$, Fig. 2i). Conversely, chemogenetic inhibition of the parvOT neurons reduced the response to airpuffs $(65 \pm 5 \%$ decrease of AUC, $p=$ 
213 Thus, we concluded that gentle non-nociceptive mechanical stimulation of the dorsal region

214 activates parvOT neurons, which we hypothesized may drive the activity of the larger population of 215 magnOT neurons.

\section{Intra-PVN connectivity of parvOT and magnOT neurons}

217 To validate this hypothesis, we first looked for direct synaptic contact of parvOT neurons onto 218 magnOT somata and/or dendrites via injection of OTp-DIO-GFP rAAV into the PVN and Cav2-Cre 219 into the SON to specifically label parvOT neurons (Extended Data Fig. 6a-b) in analogy to ${ }^{13}$. For 220 three-dimensional reconstruction of interposition between axons of parvOT neurons and somato221 dendritic domains of magnOT neurons, we employed the IMARIS technique ${ }^{26,27}$. This approach 222 allows precise identification of the location of synaptic contact by quantifying overlap with 223 synaptophysin(SYN)-immunoreactive puncta. By performing IMARIS-assisted Sholl analysis, we found synaptic-like contacts of parvOT neurons with magnOT somas and dendrites

(Fig. 3a, Extended Data Fig. 6, 6 dendritic contacts, 124 somatic contacts, n=354) as well as an average chance of innervation of $34.9 \%$ (Fig. 3b), indicating that approximately $1 / 3$ of PVN magnOT neurons receive parvOT input. Based on these anatomical observations, we performed patch-clamp recording for functional validation of parvOT-magnOT neuron connection via rAAVOTp-DIO-ChR2-mCherry (to label specifically parvOT) and rAAV-OTp-Venus (to label all OT neurons) injected into the PVN and Cav2-Cre injected into the SON (Fig. 3g). First, we confirmed the magnOT nature of recorded neurons through the presence of a hyperpolarizing transient outward rectification as well as a weak low threshold depolarization (Fig. 3h), by comparison to the 233 electrophysiological properties of identified parvOT neurons (Fig. 3c-f). We observed that 234 stimulation of parvOT neurons evoked responses in $45 \%$ of recorded magnOT neurons (9/20; Fig. 235 3i) with a significant increase of PSC frequencies (baseline $0.158 \pm 0.055 \mathrm{~Hz}$ vs ChR2 $0.346 \pm 0.15$ $\mathrm{Hz}, \mathrm{n}=9 ; \mathrm{p}<0.01$; Fig. 3i). Next, we aimed to visualize $\mathrm{Ca}^{2+}$ variations in magnOT neurons upon 
rAAV-OTp-GCaMP6s injected in the PVN and Cav2-Cre into SON (Fig 4a-d). After application of

239 CNO $(10 \mu \mathrm{M}, 1 \mathrm{~min})$, we observed that $40 \pm 8 \%$ of recorded magnOT neurons responded to 240 parvOT hM3D(Gq) stimulation, confirming again described anatomical connectivity (Fig. 3 b, i;

241 4d). In responsive neurons, the number of $\mathrm{Ca}^{2+}$ transients were significantly increased, a result 242 mirrored by the increase of areas under the curves (Fig. 4d). However, the width of these $\mathrm{Ca}^{2+}$ 243 transients did not show any significant change, indicating that parvOT-induced magnOT activity 244 does not trigger long-lasting $\mathrm{Ca}^{2+}$ transients, but several bursts of sharp $\mathrm{Ca}^{2+}$ peaks, as observed in 245 the example traces (Fig $\mathbf{4 b}$ ). This feature was further confirmed by plotting the time course of $\mathrm{Ca}^{2+}$ 246 event probability, showing that the probability of observing magnOT $\mathrm{Ca}^{2+}$ transients are increased 247 during 4 minutes after the ex vivo CNO treatment (Fig. 4c). These data indicate that parvOT 248 neurons synapse on magnOT neurons within the PVN to drive their activity, as similarly reported 249 for SON magnOT neurons in vivo ${ }^{13}$.

\section{Magnocellular neurons and their release of OT into blood are controlled by parvOT neurons}

252 Using similar viral strategies, we expressed DREADDs - hM3D(Gq) or hM4D(Gi) - specifically in 253 parvOT and injected rAAV OTp-GCaMP6s in the PVN to express the $\mathrm{Ca}^{2+}$ indicator GCaMP6s in all PVN OT neurons (1193/1371 OT neurons expressed GCaMP6s, $87 \pm 4 \%$, n = 4; Fig 4p). This allowed us to monitor the global activity of PVN OT neurons via fiber photometry in isofluraneanesthetized rats upon activation / inhibition of parvOT neurons. Activation of parvOT cells induced an increase in $\mathrm{Ca}^{2+}$ fluorescent signal of PVN OT neurons population approximately 30 min after CNO injection (i.p. $3 \mathrm{mg} / \mathrm{kg}$ ) and lasting for more than 2 hours (Fig 4e-h). Conversely, inhibition of parvOT neurons decreased $\mathrm{Ca}^{2+}$ fluorescent signals of the general population $30 \mathrm{~min}$ after CNO injection and the effect lasted for more than 2 hours (Fig 4i-l). Administration of CNO did not have any effect on $\mathrm{Ca}^{2+}$ signal in control animals lacking the DREADD receptors (Fig 4mo). Considering that the contribution of parvOT neurons to the OT population $\mathrm{Ca}^{2+}$ signal is negligible (Extended Data Fig. 7a-e), those results suggest that changes in parvOT neuron activity 
264 directly influence the firing pattern of large populations of PVN magnOT neurons. The similar

265 kinetics of $\mathrm{Ca}^{2+}$ signal fluctuations after $\mathrm{CNO}$ activation of parvOT PVN neurons together with

266 airpuff application were detected during recording of magnOT neurons in the SON, which does not

267 contain parvOT neurons (Extended Data Fig. 7f-o).

268

269 To investigate if parvOT-induced magnOT activity is followed by actual OT release, we analyzed

270 neurohypophysial OT release after chemogenetic activation of parvOT neurons. We performed

271 blood sampling from the jugular vein before and after $\mathrm{CNO}$ injection ( $3 \mathrm{mg} / \mathrm{kg}$; Fig. 4q) and found

272 a significant increase in plasma OT $45 \min (p=0.00093$ versus basal; $p=0.0036$ versus OTp-

273 mCherry control) and $90 \min (p=0.002$ versus basal; $p=0.0017$ versus OTp-mCherry control; Fig.

274 4r) after i.p. CNO injections.

275 Taken collectively, these results indicate that parvOT neurons tightly control magnOT neuron 276 activity in vivo to regulate peripheral OT release.

278 Differential neural inputs to parvOT and PVN magnOT neurons

279 The above findings suggest that parvOT neurons act as 'first responders to somatosensory input' 280 conveying information to the rest of the PVN OT neuronal population (i.e., magnOT neurons). 281 Hence, we asked whether parvOT neurons receive more synaptic inputs than PVN magnOT. In an 282 attempt to assess potential differences of synaptic inputs to parvOT and magnOT neurons, we used 283 IMARIS to quantify the total amount of SYN fluorescence at somata and dendrites. In order to 284 perform an unbiased analysis, we created spheres that precisely engulfed magnOT and parvOT 285 somata and accounted for individual variances in cell roundness and surface area (see Methods). 286 We found statistically significant differences both at the soma (Fig. 5a) and dendritic locations 287 (Fig. 5b, at two different locations, $5 \mu \mathrm{m}$ and $20 \mu \mathrm{m}$ from the soma) and analyzed a total of 104 288 neurons (parvOT=56, magnOT $=48$ ) suggesting that parvOT neurons might receive more overall 289 synaptic input. 
291 Next, to uncover the origin of synaptic inputs to parvOT and magnOT neurons, we employed the 292 retrograde trans-synaptic EnvA-pseudotyped $G$ deletion-mutant rabies virus (Rb-GFP ${ }^{28}$ ). To 293 specifically distinguish inputs to parvOT and magnOT, we used a double conditional approach, 294 which allows to retrotrace inputs to OT neurons that project to an area of choice (SON for parvOT 295 and posterior pituitary for magnOT) (see Methods, Fig. 5c,e).

In both groups of rats, we found GFP expressing neurons in numerous brain regions, including the 298 septum, medial preoptic area, and amygdala (Fig. 5d,f and Extended Data Fig. 8h), demonstrating that parvOT and magnOT neurons receive a large number of common inputs (Supplementary

Table 2). However, we detected the presence of GFP neurons in the paraventricular nucleus of thalamus, insula and habenula only after the infection of parvOT neurons (Extended Data Fig. 8i), while GFP neurons in the substantia nigra were found only in after primary infection of magnOT neurons (Extended Data Fig. 8j). In line with the IMARIS analysis, the total number of neurons projecting to parvOT and magnOT neurons was $1963.6 \pm 710$ and $694.8 \pm 121$ neurons, respectively ( $\mathrm{p}=0.02$, Fig. 5g). Although we did not find between-groups differences in the proportion of inputs coming from hypothalamic and extrahypothalamic areas (Fig. 5h), PAG and SFO showed preferential innervation of parvOT and magnOT, respectively (Fig. 5i). This indicates that parvOT neurons receive at least partially distinct, and more pronounced neuronal inputs than magnOT neurons.

\section{ParvOT neurons modulate social behavior}

312 To test whether this small population of parvOT neurons can modulate social behavior by their

313 effects on the activity of the much more abundant magnOT neurons, we used the previously

314 described chemogenetic approach to silence or activate them during behavioral tests (Fig. 6a).

315 Three weeks after viral injection, rats were injected i.p. with either CNO (3 mg/kg) or saline $60 \mathrm{~min}$ 
before social interaction tests (Fig. 6b). Selectively inhibiting the parvOT neurons resulted in less

317 social interaction: in the FSI test, the time spent with a conspecific was reduced by $37 \pm 6 \mathrm{~s}$ (over 5-

318 min sessions, $\mathrm{p}<0.001$; Supplementary Video 2). By contrast, in the CSI test, where no physical

319 contact is allowed, the time spent by the experimental rat approaching the stimulus rat was 320 unchanged (Fig. 6c-e, $\mathrm{n}=15$ rats). Conversely, CNO-induced activation of parvOT neurons led to 321 more social interaction: in the FSI test, the time spent with a conspecific increased by $10 \pm 6 \mathrm{~s}(\mathrm{p}=$ 322 0.04). In the CSI test, no significant difference in approaching time was measured between saline323 and CNO-injected rats (Fig. 6f-h, $\mathrm{n}=9$ rats).

324 Inhibition and activation of parvOT neurons also had opposite effects on crawling behavior (Fig. 6e, h). Moreover, after inhibiting parvocellular OT neurons, rats often actively avoided the stimulus rat, a behavior never observed in the control group (Extended Data Fig. 9c). Control rats injected with control virus rAAV-OTp-DIO-GFP receiving saline or CNO showed no behavioral differences (Extended Data Fig. 9a).

To control that alterations of social behaviors induced by DREADD-based manipulation of parvOT neurons activity was indeed an effect mediated by central OT release, the parvOT activation $(\mathrm{Gq})$ experiment was repeated while applying an OTR antagonist (OTR-a) ${ }^{29}$, by intracerebroventricular (i.c.v.) infusion $(0.75 \mu \mathrm{g} / 5 \mu \mathrm{l})^{30}$. As compared to saline-infused control animals, OTR-a-infused 333 animals showed a strong reduction in social interactions $(37 \pm 18 \%$ reduction, $\mathrm{p}=0.007, \mathrm{n}=12$ 334 rats), regardless of $\mathrm{CNO}$ administration, while without OTR-a, CNO application caused increased social interactions (16 $\pm 3 \%$ increase, $\mathrm{p}=0.04, \mathrm{n}=12$; Fig. 6i, Extended Data Fig. 9h). We did 336 not observe a CNO- or OTR-a-induced effect on locomotor activity (Extended Data Fig. 9b,d-e). 337 This result confirms that the downstream effect on CNO-induced activation of parvOT neurons on 338 social behavior is indeed mediated by OT and its receptors. In a second group of rats $(\mathrm{n}=10)$, 339 expressing GFP in parvOT neurons, administration of OTR-a also had a comparable effect in 
reducing social behavior; as expected, $\mathrm{CNO}$ itself did not have any effect on social interaction of 341 animals (Fig 6j, Extended Data Fig. 9i).

\section{DISCUSSION}

344 Here, we provide the first evidence that somatosensory stimulation in female rats activates parvOT

345 neurons, which subsequently drive the activation of the much larger population of magnOT

346 neurons. Using ex vivo and in vivo approaches, we demonstrated that parvOT neurons synapse onto 347 magnOT neurons to elicit a central effect of OT to promote inter-female communication.

\section{Social touch evokes OT neuronal activity}

350 The use of single unit in vivo recording precludes discrimination between parvOT and magnOT 351 neurons. However, considering limited number of parvOT neurons $\left(\sim 30\right.$ parvOT cells ${ }^{13}$ vs. $\sim 1200$ 352 magnOT cells ${ }^{31}$ in the PVN of each hemisphere) it is highly likely that we exclusively recorded 353 from magnOT cells. In support, we found that non-aggressive social interactions of female rats and, 354 in particular, physical contacts, elicited a coordinated, clustered spiking activity of PVN OT 355 neurons - a pattern that strongly facilitates activity-dependent secretion of OT from nerve terminals 356 of magnOT cells in the pituitary ${ }^{17}$ (Extended Data Fig. 2h-j). This activity is near-synchronous 357 across recorded OT neurons, and it is highly correlated with theta rhythmicity of PVN local field 358 potentials. These coordinated changes in OT neuronal electrical activity occurred only during free 359 social interaction, allowing physical contacts between conspecifics, but not during chambered social 360 interaction, where physical touch between animals was prevented by a barrier. Moreover, detailed 361 analysis of PVN OT neurons activity during social behaviors revealed that the highest increase in 362 neuronal firing occurred immediately after (0-10 s) crawling on top or being crawled behaviors 363 (Fig. 1), i.e. social contacts that involved activation of cutaneous sensory nerves. 
365 To test whether non-noxious repetitive somatosensory stimulations directly influence PVN OT

366 neurons activity, even in the absence of other stimuli, we applied 'airpuff' stimulations onto the

367 skin of the dorsal area of the rat, in lightly anesthetized conditions, while measuring action

368 potentials of PVN neurons. Notably, 'airpuffs' induced a significant increase in spiking activity of

369 most (83\%) recorded putative magnOT neurons but had little or no effect on the activity of non-OT

370 PVN neurons, reinforcing the idea that somatosensory inputs selectively activate magnOT neurons.

371 This finding is in line with previous studies ${ }^{32}$ that reported increased OT plasma levels in rats after

37210 minutes of massage-like stroking. Furthermore, the stimulation of low-threshold

373 mechanoreceptors, particularly the touch-sensitive nerve fibers C-tactile afferents is known to

374 trigger OT release and has been associated with increased social motivation in rodents and human ${ }^{33}$.

\section{ParvOT neurons control PVN magnOT neuron activity}

377 To shed light on the causal link between somatosensory stimulation and social behavior, we focused

378 our research on a specific subtype of 'parvocellular' OT neurons. These neurons communicate with

379 various autonomic centers in the brain stem and spinal $\operatorname{cord}^{20}$, and are involved in analgesia during

380 acute pain ${ }^{13}$.

382 When we applied low-intensity, non-noxious cutaneous stimulation (airpuff) in awake rats, we observed a sustained increase of $c$-fos expression in parvOT neurons in the PVN (Fig. 2). As of

384 note, we found that airpuff induced $c$-fos expression in parvOT, but not in magnOT neurons.

385 However, the absence of $c$-fos expression in magnOT cells does not necessarily indicate the absence of their increasing activity ${ }^{34-36}$. Indeed, only dramatic physiological challenges such as hemorrhage,

387 salt loading, or fear evoke $c$-fos expression in magnOT neurons ${ }^{34,37}$. Importantly, during lactation magnOT neurons release a large amount of OT into peripheral circulation while an increase in $c$-fos expression was never found. In analogy, our findings demonstrate increased OT plasma

390 concentrations after chemogenetic activation of parvOT neurons (Fig. 4) via a demonstrated 
parvOT $\rightarrow$ magnOT connectivity, although without detectable $c$-fos immunosignal in magnOT

392 neurons releasing the neuropeptide into the blood.

393

394 Further, chemogenetic activation or inhibition of parvOT neurons via DREADDs resulted, 395 respectively, in an increase or decrease in OT neuron activity in response to the air puff stimulation

396 (Fig. 2). This suggests that parvOT neurons can be activated by both nociceptive ${ }^{13}$ and non397 nociceptive stimuli (present study) and subsequently promoting analgesia as well as social behavior. 398 Such pleotropic effects of OT originating from the same parvOT neurons require further 399 investigation.

400

401 To provide additional evidence that parvOT neurons modulate magnOT neuron activity within the 402 PVN, we employed a combination of immunohistochemistry and three-dimensional anatomical 403 reconstruction. We found 1.5-4-fold more synaptic-likes contacts on ParvOT somas and dendrites 404 compared to respective compartments of MagnOT neurons (Fig. 5). This finding is supported by 405 retrograde tracing data, which demonstrate substantially more inputs to parvOT neurons than 406 to magnOT neurons (Fig. 5).

407

408 Do parvOT neurons control social behavior?

409 To investigate how parvOT neurons modulate social behavior, we performed chemogenetic 410 manipulation of parvOT neurons by viral means. We found that targeted activation or inhibition of 411 parvOT neurons increased or decreased the total time of social interaction with a conspecific, 412 respectively. Further, the i.c.v. application of an OTR antagonist prevented CNO-induced social 413 interaction after chemogenetic activation of parvOT neurons (Fig. 6). This suggests that the 414 excitation of parvOT neurons is transmitted to magnOT cells, which, in turn, project axonal 415 collaterals to numerous forebrain brain regions ${ }^{5,7}$. Given that parvOT neurons exclusively project to 416 the brainstem and spinal cord ${ }^{38}$, our results allow to hypothesize that the OTR antagonist blocks the 
417 action of OT released from magnOT axons in socially-relevant brain regions, resulting in the

418 attenuation of social communication between female conspecifics.

\section{A stable OT-mediated social interaction throughout a female life?}

421 While we exclusively used virgin females in our current study, it will be important to investigate 422 how pregnancy and lactation changes the OT-dependent response to somatosensory stimulation. 423 Given the drastic activation of the OT system and close physical contact with the offspring 424 peripartum ${ }^{39-41}$, it is plausible that the reward of tactile stimulation changes as well. Moreover, due 425 to the interaction of OT and prolactin during the milk letdown reflex ${ }^{42,43}$, nipples might become more sensitive to the suckling of pups, which might translate into a more rewarding experience for mothers. Further studies are needed to assess the intricate relationship between social touch, social behavior and social motivation, which requires concomitant actions of OT, serotonin, and dopamine within the nucleus accumbens and ventral tegmental area ${ }^{22,44}$. Accordingly, we found that parvOT, but not magnOT neurons, are innervated by neurons of the insular cortex, which is critical region processing social touch ${ }^{45}$, and could thus be potentially involved in the recruitment of the OT-ergic system during social tactile stimulation.

Taken together, our data extend the current knowledge of the relationship between intracerebral OT release, social touch and its behavioral correlates. Our results suggest that parvOT neurons translate mechanosensory information from the periphery into social behavior (Extended Data Fig. 10), but the precise ascending pathways from cutaneous nerves - via the parvOT $\rightarrow$ magnOT circuit - to forebrain regions controlling social behaviors await further investigation.

While intranasal OT application has improved clinical outcomes of schizophrenia, posttraumatic stress disorders and autism spectrum disorders, there is still an ongoing debate about the validity of 442 these findings ${ }^{46}$, suggesting that evoking endogenous OT release might be a more reliable way to 
443 exploit the benefits of this neuropeptide. Thus, a combination of gentle touch, social interaction

444 and/or intranasal OT application might be a powerful tool to treat human mental diseases, in which 445 the OT system is compromised ${ }^{47,48}$.

446

447

\section{References}

448 1. Lee, H.-J., Macbeth, A. H., Pagani, J. \& Scott Young 3rd, W. Oxytocin : the great facilitator $449 \quad$ of life. Prog. Neurobiol 88, 127-151 (2010).

450 2. Jurek, B. \& Neumann, I. D. The oxytocin receptor: from intracellular signaling to behavior. $451 \quad$ Physiol. Rev. 98, 1805-1908 (2018).

452 3. Walum, H. \& Young, L. J. The neural mechanisms and circuitry of the pair bond. Nat. Rev. $453 \quad$ Neurosci. 19, 643-654 (2018).

454 4. Russell, J. A., Leng, G. \& Douglas, A. J. The magnocellular oxytocin system, the fount of 455 maternity: adaptations in pregnancy. Front. Neuroendocrinol. 24, 27-61 (2003).

456 5. Knobloch, H. S. et al. Evoked axonal oxytocin release in the central amygdala attenuates fear 457 response. Neuron 73, 553-566 (2012).

458 6. Marlin, B. J. \& Froemke, R. C. Oxytocin modulation of neural circuits for social behavior. 459 Dev. Neurobiol. 77, 169-189 (2017).

460 7. Grinevich, V. \& Stoop, R. Interplay between oxytocin and sensory systems in the 461 orchestration of socio-emotional behaviors. Neuron 99, 887-904 (2018).

462 8. Dumais, K. M., Alonso, A. G., Immormino, M. A., Bredewold, R. \& Veenema, A. H. 463 Involvement of the oxytocin system in the bed nucleus of the stria terminalis in the sex464 specific regulation of social recognition. Psychoneuroendocrinology 64, 79-88 (2016).

465 9. Dumais, K. M., Alonso, A. G., Bredewold, R. \& Veenema, A. H. Role of the oxytocin 466 system in amygdala subregions in the regulation of social interest in male and female rats. $467 \quad$ Neuroscience 330, 138-149 (2016).

468 10. Resendez, S. L. et al. Social stimuli induce activation of oxytocin neurons within the 
paraventricular nucleus of the hypothalamus to promote social behavior in male mice. $J$. Neurosci. 40, 2282-2295 (2020).

471 11. Bobrov, E., Wolfe, J., Rao, R. P. \& Brecht, M. The representation of social facial touch in rat 472 barrel cortex. Curr. Biol. 24, 109-115 (2014).

473

474

475

476

477

478

479

480

12. Chen, P. \& Hong, W. Neural circuit mechanisms of social behavior. Neuron 98, 16-30 (2018).

13. Eliava, M. et al. A new population of parvocellular oxytocin neurons controlling magnocellular neuron activity and inflammatory pain processing. Neuron 89, 1291-1304 (2016).

14. Lima, S. Q., Hromádka, T., Znamenskiy, P. \& Zador, A. M. PINP: a new method of tagging neuronal populations for identification during in vivo electrophysiological recording. PLoS One 4, e6099 (2009).

15. Leng, T., Leng, G. \& MacGregor, D. J. Spike patterning in oxytocin neurons: Capturing physiological behaviour with Hodgkin-Huxley and integrate-and-fire models. PLoS One 12, e0180368 (2017)

16. Netser, S., Haskal, S., Magalnik, H. \& Wagner, S. A novel system for tracking social preference dynamics in mice reveals sex- and strain-specific characteristics. Mol. Autism 8, 53 (2017).

17. Maícas-Royo, J., Leng, G. \& MacGregor, D. J. A predictive, quantitative model of spiking activity and stimulus-secretion coupling in oxytocin neurons. Endocrinology 159, 1433-1452 (2018).

18. Portfors, C. V. Types and functions of ultrasonic vocalizations in laboratory rats and mice. $J$. Am. Assoc. Lab. Anim. Sci. 46, 28-34 (2007).

19. Lenschow, C. et al. Sexually monomorphic maps and dimorphic responses in rat genital cortex. Curr. Biol. 26, 106-113 (2016).

20. Althammer, F. \& Grinevich, V. Diversity of oxytocin neurones: Beyond magno- and 
parvocellular cell types? J. Neuroendocrinol. 30, e12549 (2018).

21. Johnson, Z. V. et al. Central oxytocin receptors mediate mating-induced partner preferences and enhance correlated activation across forebrain nuclei in male prairie voles. Horm. Behav. 79, 8-17 (2016).

22. Hung, L. W. et al. Gating of social reward by oxytocin in the ventral tegmental area. Science (80-. ). 357, 1406-1411 (2017).

23. Okabe, S., Yoshida, M., Takayanagi, Y. \& Onaka, T. Activation of hypothalamic oxytocin neurons following tactile stimuli in rats. Neurosci. Lett. 600, 22-27 (2015).

24. Bru, T., Salinas, S. \& Kremer, E. J. An Update on Canine Adenovirus Type 2 and Its 504 Vectors. Viruses 2, 2134-2153 (2010).

25. Gunaydin, L. A. et al. Natural neural projection dynamics underlying social behavior. Cell 157, 1535-1551 (2014).

26. VanRyzin, J. W. et al. Microglial phagocytosis of newborn cells is induced by endocannabinoids and sculpts sex differences in juvenile rat social play. Neuron 102, 435-

27. Erny, D. et al. Host microbiota constantly control maturation and function of microglia in the CNS. Nat. Neurosci. 18, 965-977 (2015).

28. Wickersham, I. R. et al. Monosynaptic restriction of transsynaptic tracing from single,

30. Grund, T. et al. Neuropeptide $\mathrm{S}$ activates paraventricular oxytocin neurons to induce anxiolysis. J. Neurosci. 37, 12214-12225 (2017).

31. Rhodes, C. H., Morriell, J. I. \& Pfaff, D. W. Immunohistochemical analysis of magnocellular elements in rat hypothalamus: Distribution and numbers of cells containing neurophysin, 
521 32. Uvnäs-Moberg, K., Handlin, L. \& Petersson, M. Self-soothing behaviors with particular

522

523

524

525

526

527

528

529

530

531

532

533

534

535

536

537

538

539

540 reference to oxytocin release induced by non-noxious sensory stimulation. Front. Psychol. 5, $1529(2015)$.

33. Walker, S. C., Trotter, P. D., Swaney, W. T., Marshall, A. \& Mcglone, F. P. C-tactile afferents: Cutaneous mediators of oxytocin release during affiliative tactile interactions? Neuropeptides 64, 27-38 (2017).

34. Brown, C. H., Bains, J. S., Ludwig, M. \& Stern, J. E. Physiological regulation of magnocellular neurosecretory cell activity: integration of intrinsic, local and afferent mechanisms. J. Neuroendocrinol. 25, 678-710 (2013).

35. Hoffman, G. E. \& Lyo, D. Anatomical markers of activity in neuroendocrine systems: are we all 'Fos-ed out'? J. Neuroendocrinol. 14, 259-268 (2002).

36. Hoffman, G. E., Smith, M. S. \& Verbalis, J. G. c-Fos and related Immediate Early Gene products as markers of activity in neuroendocrine systems. Front. Neuroendocrinol. 14, 173$213(1993)$.

37. Hasan, M. T. et al. A fear memory engram and its plasticity in the hypothalamic oxytocin system. Neuron 103, 133-146.e8 (2019).

38. Stern, J. E. Electrophysiological and morphological properties of pre-autonomic neurones in the rat hypothalamic paraventricular nucleus. J. Physiol. 537, 161-177 (2001).

39. Bosch, O. J. Brain Oxytocin Correlates with Maternal Aggression: Link to Anxiety. J. Neurosci. 25, 6807-6815 (2005).

40. Fenelon, V. S., Poulain, D. A. \& Theodosis, D. T. Fos synthesis and neuronal activation: analysis of Fos immunoreactivity in identified magnocellular neurons during lactation. Ann. N. Y. Acad. Sci. 689, 508-511 (1993).

41. Neumann, I., Douglas, A. J., Pittman, Q. J., Russell, J. A. \& Landgraf, R. Oxytocin released within the supraoptic nucleus of the rat brain by positive feedback action is involved in parturition-related events. J. Neuroendocrinol. 8, 227-233 (1996). 
42. Augustine, R. A. et al. Prolactin regulation of oxytocin neurone activity in pregnancy and lactation. J. Physiol. 595, 3591-3605 (2017).

43. Kennett, J. E. \& McKee, D. T. Oxytocin: an emerging regulator of prolactin secretion in the female rat. J. Neuroendocrinol. 24, 403-412 (2012).

44. Dölen, G., Darvishzadeh, A., Huang, K. W. \& Malenka, R. C. Social reward requires coordinated activity of nucleus accumbens oxytocin and serotonin. Nature 501, 179-184 (2013).

45. McGlone, F., Wessberg, J. \& Olausson, H. Discriminative and affective touch: sensing and feeling. Neuron 82, 737-755 (2014).

46. Leng, G. \& Ludwig, M. Reply to: Improving research standards to restore trust in intranasal oxytocin. Biol. Psychiatry 79, e55-e56 (2016).

47. Meyer-Lindenberg, A., Domes, G., Kirsch, P. \& Heinrichs, M. Oxytocin and vasopressin in the human brain: social neuropeptides for translational medicine. Nat. Rev. Neurosci. 12, $524-538$ (2011).

48. Grinevich, V. \& Neumann, I. D. How puzzle stones from animal studies translate into psychiatry. Mol. Psychiatry (2020). doi:10.1038/s41380-020-0802-9

\section{ACKNOWLEDGEMENTS}

The authors thank Thomas Grund and Xinying Liu for initial contribution to this study, Ron Stoop for valuable comments on the manuscript, Judith Müller for packaging viral vectors, Eric Kremer for the canine virus, Jorge Maicos-Roya for contributing to the modeling of OT release, Shai Netser for his comments on the manuscript, Claudia Pitzer and the Interdisciplinary Neurobehavioral Core Facility of Heidelberg University for some of behavioral experiments performed there, and Thomas Splettstoesser (www.scistyle.com) for composing Figure 7. The work was supported by German Research Foundation (DFG) within the Collaborative Research Center (SFB) 1158 seed grant for 
573 young researchers (to AL), DFG postdoctoral fellowship AL 2466/1-1 (to FA), Alexander von

574 Humboldt research fellowship (to DH), Human Frontier Science Program RGP0019/2015 (to VG

575 and SW), Israel Science Foundation grants \#1350/12, 1361/17, Milgrom Foundation and the

576 Ministry of Science, Technology and Space of Israel grant \#3-12068 (to SW), NIH grant

577 R01NS094640 (to JES), BBSRC grant BB/S000224/1 (to GL), DFG grants NE 465/27, NE 465/31, 578 and NE 465/34 (to IDN), ANR-DFG grant GR 3619/701 (to AC and VG), NARSAD Young 579 Investigator Grant 24821 and ANR JCJC grant (to AC), DFG grant GR 3619/4-1, SFB 1158, 580 SNSF-DFG grant GR 3619/8-1, and Fritz Thyssen Foundation grant 10.16.2.018 MN (to VG).

\section{AUTHORS CONTRIBUTIONS}

583 Design and project conception (YT, DB, AL, AC, VG), ex vivo electrophysiology (LH, PD), ex vivo 584 calcium imaging ( $\mathrm{LH}, \mathrm{ABa})$, in vivo electrophysiology (YT, DB, SW), fiber photometry (YT, AL), 585 behavioral experiments and analyses (YT, DB, SW), immunohistochemistry and confocal 586 microscopy (DB, ME, DH, FA), trans-synaptic labelling of OT neurons inputs (AL, JS), assistance 587 with viruses design for trans-synaptic labelling (MS, MO, KKC), three-dimensional reconstruction 588 and analysis (FA, MKK, RKR, JES), plasma OT dosages (ABl, IDN), modeling (GL), manuscript 589 preparation (YT, DB, AL, LH, FA, IDN, AC, VG), supervision, project administration, and funding 590 acquisition (IDN, AC, VG).

591

592 CONFLICT OF INTEREST

593 The authors declare no competing interests.

594

595

596

597

598 


\section{FIGURE LEGENDS}

600

601

\section{Figure 1. In vivo recording of individual OT neurons in the PVN}

602

a, Setup for recordings of behavior, ultrasonic vocalizations, and neural activity.

603

b, Video-tracking and electrophysiological recording from a rat alone in the OF arena (top) and

604

during FSI (bottom): animal movement path (blue line), location of prominent OT cell activity

605

(colored dots), heatmap of time spent by the rat in different locations.

606

c, Example firing rate of four identified OT neurons recorded simultaneously during FSI. Red bars

607 indicate periods of social interaction.

608

d, Average firing rate of 15 OT neurons from five rats: OF baseline $1.1 \pm 0.4 \mathrm{~Hz}$, not socially interacting (not SI) $1.6 \pm 0.3 \mathrm{~Hz}$, and social interacting (SI) $2.6 \pm 0.2 \mathrm{~Hz}(\mathrm{OF}-$ not SI p $=0.07$, OF-SI $\mathrm{p}=0.001$, not SI-SI $\mathrm{p}=0.03$, one-way ANOVA). Average index of dispersion on 1-s time bins of 15 OT neurons: OF $0.9 \pm 0.2$, not SI $1.4 \pm 0.3$, SI $3.4 \pm 0.4($ OF-not SI $p=0.16$, OF-SI $p=0.0004$ not SI-SI $p=0.001$, one-way ANOVA). Average pairwise Pearson correlation of spiking activity

613 (1-s time bins) of 17 OT neurons' pairs recorded in OF and SI ( $\mathrm{p}=0.005$, unpaired two-sided t 614 test).

615 e, Frames of recorded videos (top) of experimental rats that were placed either alone (OF), or with a 616 mesh between rats (CSI) or for FSI with a stimulus rat; representative spike raster plots of an OT 617 cell in each condition (bottom).

618 f, Average firing rate of 15 OT neurons while rats underwent open field (OF), CSI, and FSI tests 619 (OF-CSI $\mathrm{p}=0.14$, OF-FSI $\mathrm{p}=0.004$, CSI-FSI $\mathrm{p}=0.006, \mathrm{n}=15$ cells, one-way ANOVA). Average 620 index of dispersion on 1 -s time bins (OF-CSI $p=0.21$, OF-FSI $p=0.001$, CSI-FSI $p=0.003, n=15$ 621 cells, one-way ANOVA). Average pairwise Pearson correlation of spiking activity (1-s time bins) 622 of 17 OT neurons' pairs (OF-CSI $\mathrm{p}=0.39$, OF-FSI $\mathrm{p}=0.002$, CSI-FSI $\mathrm{p}=0.003$, one-way 623 ANOVA). 
624 g, Normalized firing rates of OT neurons during each behavior; crawling on top and being crawled 625 elicited the strongest responses $\left(* \mathrm{p}=0.036,{ }^{*} \mathrm{p}=0.024, \mathrm{n}=8\right.$ cells, one-way ANOVA followed by 626 Tukey's post hoc test).

627 h, Representative spike raster plots, averaged response, and PSTH of OT cell activity during 628 'crawling on top' (increased response, $\mathrm{p}=0.036, \mathrm{n}=6$ cells, Wilcoxon test), and 'being crawled' 629 (increased response, $\mathrm{p}=0.024, \mathrm{n}=6$ cells, Wilcoxon test) behaviors. Data represented as mean \pm 630 SEM.

631 Figure 2. Gentle non-nociceptive mechanical stimuli triggers OT neurons activation

632 a, Head-fixed rats injected with rAAV-pOT-ChR2-mCherry were stimulated with airpuffs at 633 anterior, central, and posterior portion of the dorsal body region, while OT neurons were recorded 634 with an opto-electrode. Top: PSTHs example of OT neurons responses to airpuffs. Bottom: 635 normalized PSTHs of 10 (out of 23) recorded OT neurons response to airpuffs in three dorsal body 636 regions ( 1 anterior, 2 central, 3 posterior); red indicate high spiking activity.

637 b, (Top) Statistics of average firing rate of OT neurons response to airpuff stimulations (peak vs. 638 baseline, $* \mathrm{p}=0.017,{ }^{*} \mathrm{p}=0.025,{ }^{*} \mathrm{p}=0.021, \mathrm{n}=23$ cells from 8 rats, one-way ANOVA followed 639 by Bonferroni post hoc comparison) indicates significant increase above basal rate (dashed line). 640 (Bottom) latency of OT neurons responses to airpuffs. All data show average \pm SEM.

641 c, Fluorogold-injected rats received continuous airpuffs for $10 \mathrm{~min}$ and were killed and perfuse642 fixed 90 min later. PVN slices were triple-stained with antibodies against OT (blue), fluorogold 643 (red) and c-fos (green). The confocal image shows a (fluorogold-negative) parvocellular OT neuron 644 expressing $c$-fos (one of 99 such double-labeled neurons observed in 4 rats). Scale bars $=100$ and 64510 (inset) $\mu \mathrm{m}$.

646 d, Rats injected bilaterally with CAV2-Cre into the SON and rAAV-OTp-DIO-mCherry into the 647 PVN, were exposed to airpuffs for $10 \mathrm{~min}$ and killed $90 \mathrm{~min}$ later. The confocal image shows $c$-fos 
expression in a parvOT neuron (mCherry-positive, labeled via the retrograde CAV2-Cre, and is one of 60 such triple-labeled neurons observed in 4 rats). Scale bars $=100$ and 10 (inset) $\mu \mathrm{m}$. e-f, Viral vectors for recording $\mathrm{Ca}^{2+}$ signals in GCaMP6s-expressing OTneurons during chemogenetic activation (e) or silencing (f) of parvocellular OTneurons.

g-h, Examples of fiber photometry-based $\mathrm{Ca}^{2+}$ signals of PVN OT neuron population during airpuff stimulation (orange bars). Top: response to airpuffs 30-60 min after saline injection (Control); bottom: response to airpuffs 30-60 min after $\mathrm{CNO}$ induced activation (g) or silencing (h) of parvOT neurons.

i, Average traces of $\mathrm{Ca}^{2+}$ responses to airpuffs 30-60 min after injection of CNO to activate (Gq) parvOT neurons, or saline (Control). Each graphic is the average of 33 airpuffs responses (11 airpuffs per animal, $n=3$ ); area under the curve (AUC) 0-30 s after airpuffs, relative to control (* $\mathrm{p}$ $=0.03$, paired two-sided $\mathrm{t}$ test).

j, Average traces of $\mathrm{Ca}^{2+}$ responses to airpuffs 30-60 min after injection of $\mathrm{CNO}$ to silence (Gi) parvOT neurons, or of saline (Control). Each graphic is the average of 33 airpuffs responses (11 airpuffs per animal, $\mathrm{n}=3$ ); area under the curve (AUC) 0-30 s after airpuffs, relative to control (** $\mathrm{p}=0.007$, paired two-sided t test). All data show average \pm SEM.

\section{Figure 3. Intra-PVN connectivity of parvOT and magnOT neurons}

a, Images show the three-dimensional surface reconstruction of OT, GFP and SYN. Circles with dashed lines indicate the overlap of OT, GFP and SYN.

b, Confocal image shows a single magnOT neuron (purple) innervated by a parvOT fiber (green). Scale bar $=10 \mu \mathrm{m}$. Dot plot graph shows that the chance of innervation by parvOT neurons depends on the anatomical location of magnOT neurons within the PVN. Bar graph shows the average chance for magnOT PVN neurons to be innervated by parvOT axons ( $\mathrm{n}=214$ cells from 3 rats). 
c, Schema of the viral injection into the SON and the PVN plus the electrophysiological recording

672 in the PVN (with pipette) for the recording of parvOT neurons (expressing mCherry + GFP) and

673 magnOT neurons (expressing mCherry).

674 d, Comparison of average and individual points of voltage amplitude between parvOT neurons $(\mathrm{n}=$ 67517 cells from 4 rats) and magnOT neurons $(n=7$ cells from 4 rats), for different 676 electrophysiological parameters (AP; parvOT $70.12 \pm 2.87 \mathrm{mV}$ vs magnOT $71.65 \pm 7.414 \mathrm{mV} ; \mathrm{p}=$ 677 0.82, unpaired two-sided t test; transient outward rectification (TOR); magnOT $=4.39 \pm 0.79 \mathrm{mV}$; 678 low threshold depolarization (LTD); parvOT $14.88 \pm 0.81 \mathrm{mV}$ vs magnOT $5.93 \pm 1.98 \mathrm{mV} ; * * \mathrm{p}=$ 679 0.0019, two-sided Mann-Whitney U test).

680 e, Example responses of 3 parvOT neurons to a hyperpolarizing current at $-100 \mathrm{pA}$ followed by 4 681 current injections starting from $0 \mathrm{pA}$ to $60 \mathrm{pA}$.

$682 \mathrm{f}$, Example responses of 3 magnOT neurons to a hyperpolarizing current at $-100 \mathrm{pA}$ followed by 4 683 current injections starting from $0 \mathrm{pA}$ to $60 \mathrm{pA}$.

$684 \mathrm{~g}$, (left) Schematic representation of viral vectors injected in the PVN (OTp-DIO-ChR2-mCherry 685 and OTp-Venus) and in the SON (CAV2-Cre) to transduce the expression of ChR2-mCherry in 686 parvOT neurons and of Venus in PVN OT neurons; (right) image showing viral expression in the 687 PVN in one out of 4 rats (scale bar $=100 \mu \mathrm{m})$.

$688 \mathrm{~h}$, Average and individual points of voltage amplitude of magnOT neurons ( $\mathrm{n}=8$ cells from 4 rats), 689 for different electrophysiological parameters: AP, TOR, and LTD.

$690 \mathrm{i}$, Average percentage ( $45 \%)$ of responding magnOT neurons $(\mathrm{n}=9$ cells) in all the magnOT 691 neurons that have been recorded ( $\mathrm{n}=20$ cells from 4 rats). MagnOT PSC frequency reversibly 692 increases after parvOT ChR2 photostimulation $(\mathrm{n}=9$ cells). Example responses of 3 magnOT 693 neurons in voltage clamp configuration at $-70 \mathrm{mV}$ before and after the $\mathrm{ChR} 2$ optogenetic 694 stimulation of parvOT neurons. Baseline vs. BL ${ }^{* *} \mathrm{p}<0.001$, BL vs. wash ${ }^{* *} \mathrm{p}<0.001$, Friedman 695 test followed by a Dunn post hoc test. All data are represented as mean + SEM. 
a, To allow the expression of $\mathrm{hM} 3 \mathrm{D}(\mathrm{Gq})$ on parvOT PVN to SON projecting neurons, rats' SON have been infected with a CAV2-Cre rAAV and the PVN were infected with an AAV allowing the

Cre-dependent expression of $\mathrm{hM} 3 \mathrm{D}(\mathrm{Gq})$ under the control of the OT promoter. We also make PVN OT neuron express the calcium indicator GCaMP6s to monitor calcium transients in parvOT neurons.

b, Example traces of the effect of CNO $(10 \mu \mathrm{M}, 6 \mathrm{~min})$ on PVN OTergic neurons calcium activity.

c-d, CNO application increase the number of calcium transients by $5 \pm 1$ fold (solid line: average, shaded area: SEM, $p=0.0019$, Wilcoxon test) and the AUC by $15 \pm 9$ fold $(p=0.0043$, Wilcoxon test) in $40 \pm 8 \%$ of recorded magnOT neurons $(n=20$ slices from 7 rats, 70 cells $)$. After CNO application, the probability of observing a calcium peak is increased during $\sim 4$ minutes but the duration of those peaks remains unchanged (ratio $=2 \pm 0.7, \mathrm{p}=0.46$, paired two-sided $\mathrm{t}$ test). Bar 710 plots show mean + SEM.

711 e-h, Schema of viral vectors injected and implanted optic fiber for fiber photometry recording (e) of PVN OT neurons with concomitant DREADD-Gq activation of parvOT neurons. Example traces (f) parvOT neurons. Normalized area under the curve (AUC) of GCaMP6s signal (g, solid line: average, shaded area: SEM, 1 min bin size) of PVN OT neurons showing increase of cellular activity after parvOT activation mediated by $\mathrm{CNO}$ i.p. injection (indicated by arrow). 30-min averaged AUC (h) showing a gradual increase of cellular activity (baseline AUC vs 0-30 min p = 0.0606; vs $30-60 \min * \mathrm{p}=0.0403$ ) that last at least 120 min (baseline AUC vs $60-90 \mathrm{~min} * \mathrm{p}=$ 0.028; vs $90-120 \min * \mathrm{p}=0.0325, \mathrm{n}=6$ rats, two-way ANOVA Tukey's corrected post-hoc comparison).

i-l, Schema of viral vectors injected and implanted optic fiber for fiber photometry recording (i) of PVN OT neurons with concomitant DREADD-Gi inhibition of parvOT neurons. Example traces (j) 
of recorded GCaMP6s signal from PVN OT neurons before and after CNO-induced inhibition of

724 parvOT neurons. Normalized area under the curve (AUC) of GCaMP6s signal (k, solid line:

725 average, shaded area: SEM, 1 min bin size) of PVN OT neurons showing decrease of cellular

726 activity after parvOT inhibition mediated by i.p. CNO injection (indicated by arrow). 30-min

727 averaged AUC (l) showing a gradual decrease of cellular activity (baseline AUC vs 0-30 min $\mathrm{p}=$

7280.058 ; vs $30-60 \min * * * \mathrm{p}=0.00013$ ) that last at least $120 \mathrm{~min}$ (baseline AUC vs 60-90 min, 90-120

$729 \min * * * \mathrm{p}=0.00019, \mathrm{n}=3$ rats, two-way ANOVA Tukey's corrected post-hoc comparison).

$730 \mathbf{m - o}$, Schema of viral vectors injected and implanted optic fiber for fiber photometry recording (m)

731 of PVN OT neurons in control animals (DREADD-free) expressing GFP in parvOT neurons.

732 Normalized area under the curve (AUC) of GCaMP6s signal (n, solid line: average, shaded area:

733 SEM, 1 min bin size) of PVN OT neurons showing no significant changes in $\mathrm{Ca}^{2+}$ signal upon CNO

734 injection. No significant changes are detected in 30-min averaged AUC (o) up to $120 \min (\mathrm{p}=$

$7350.109, \mathrm{n}=2$ rats, two-way ANOVA Tukey’s corrected post-hoc comparison).

736 p, Panels of immunostained section of the PVN showing post-hoc verification of implanted optic

737 fiber above the PVN and co-localization of immunoreactive GCaMP6s (green, top left), and DIO-

$738 \mathrm{hM} 3 \mathrm{D}(\mathrm{Gq})$-mCherry (red, bottom left), and oxytocin (blue, right) in one out of six rats. Arrows

739 indicate mCherry-positive parvOT neurons. Scale bar $100 \mu \mathrm{m}$ and $10 \mu \mathrm{m}$ (inset).

740 q, Schema of viral vectors injected for DREADD-Gq activation of parvOT neurons and blood 741 sampling from jugular vein.

742 r, Chemogenetic activation of parvOT neurons evokes peripheral OT release. Plasma OT (pg/ml)

743 taken under basal conditions and 45 as well as $90 \mathrm{~min}$ after i.p. CNO (3 mg/kg; depicted by arrow),

$744 \mathrm{n}=8$ rats parvOT Gq group, $\mathrm{n}=6$ rats control group. At $45 \mathrm{~min},++\mathrm{p}=0.00093$ vs. basal $(-45$

$745 \mathrm{~min}),{ }^{* *} \mathrm{p}=0.0036$ vs. control (OTp-mCherry) and at $90 \mathrm{~min}, \mathrm{p}=0.002$ vs. basal, $\mathrm{p}=0.0017$ vs

746 control. Two-way ANOVA for repeated measures, Bonferroni corrected post-hoc. Data are 747 presented as mean \pm SEM. 


\section{Figure 5. ParvOT neurons receive more inputs than magnOT neurons}

750 a, Three-dimensional reconstruction of parvOT and magnOT neurons and the quantification of

751 SYN fluorescence. Asterisks (white) indicate the placement of the spheres (yellow) used to quantify

752 the total amount of SYN fluorescence (red). Top panel shows the placement of a sphere around a 753 magnOT neuron soma, bottom panel shows the placement of a sphere onto a parvOT neuron 754 dendrite. Scale bars $=5 \mu \mathrm{m}$.

755 b, Quantification of SYN fluorescence in close proximity to parvOT ( $\mathrm{n}=56$ cells from 3 rats) and 756 magnOT ( $\mathrm{n}=48$ cells from 3 rats) neurons at somatic (top graph) and dendritic locations (bottom 757 graph) considering differences in cellular roundness and surface area (adjusted). Unpaired two758 sided t test, $* * * \mathrm{p}<0.0001$

759 c, e, Virus injection strategy to retrotrace inputs from parvOT and magnOT neurons, respectively.

760 d, f, Schema representing the proportion of inputs (number of inputs from one brain area / total 761 number of inputs) from each brain area to parvOT and magnOT neurons, respectively. Brain areas 762 projecting only to parvOT or magnOT are circled in green or purple, respectively.

$763 \mathrm{~g}$, Quantification of the total number of inputs to parvOT and magnOT neurons. Two-sided t test, $764 * \mathrm{p}=0.0223, \mathrm{n}=5$ rats per group.

765 h, Proportion of inputs to parvOT and magnOT neurons located in the hypothalamus or outside the 766 hypothalamus. Numbers indicate average number of neurons.

767 i, Bar graphs showing the proportion of inputs coming from brain areas showing preferential 768 innervation of parvOT or magnOT. Two-sided t test, asterisks indicate significant difference: ${ }^{*} \mathrm{p}=$ $7690.0315,{ }^{*} \mathrm{p}=0.0153,{ }^{*} \mathrm{p}=0.0264,{ }^{*} \mathrm{p}=0.0299,{ }^{*} \mathrm{p}=0.0453$ and ${ }^{*} \mathrm{p}=0.0011$ respectively, $\mathrm{n}=5$ rats 770 per group. Data represented as mean + SEM.

771 parvOT neurons. 
b, $\mathrm{CNO}$ or saline was injected i.p. 60 min before the behavioral tests.

776 c, Silencing parvOT neurons (Parvo-Gi group): percentage of time spent by an experimental rat

777

778

779

780

781

782

783

784

785

786

787

788

789

790

791

792

793

794

795

796

797

798

799

800 injected with saline or CNO socially interacting with a conspecific in CSI $(p=0.41)$ and FSI $(n=$ 15 rats, $* * * p=0.0001$, paired two-sided t test), calculated over the 5 -min session.

d, Temporal dynamics of time spent in social interaction in $1-\min$ bins $\left(2^{\text {nd }}\right.$ minute, $* * p=0.01,3^{\text {rd }}$ minute $* \mathrm{p}=0.03,5^{\text {th }}$ minute $* \mathrm{p}=0.04, \mathrm{n}=15$ rats, two-way ANOVA time $\mathrm{x}$ treatment).

e, Parvo-Gi group: time spent in different social behaviors in rats injected with saline or CNO: crawling on top $(* * p=0.008)$, sniffing $(* p=0.012)$, chasing $(p=0.13)$, head-to-head $(p=0.31), n$ $=15$ rats, one-way ANOVA Tukey's corrected post-hoc comparison.

f, Activation of parvOT neurons (Parvo-Gq group): average time spent in social interaction with conspecific stimulus in CSI $(\mathrm{p}=0.32)$ and FSI $\left(\mathrm{n}=9\right.$ rats, ${ }^{*} \mathrm{p}=0.04$, paired two-sided t test $)$ after CNO or saline injection.

g, Temporal dynamics of time spent in social interaction in 1-min bins for rats injected with CNO or saline ( $4^{\text {th }}$ minute, $\mathrm{p}=0.03,5^{\text {th }}$ minute, $\mathrm{n}=9$ rats, $\mathrm{p}=0.05$, two-way ANOVA time $\mathrm{x}$ treatment).

h, Parvo-Gq group: time spent in different social behaviors in rats injected with saline or CNO: mounting $(* * p=0.006)$, sniffing $(\mathrm{p}=0.44)$, chasing $(\mathrm{p}=0.27)$, head-to-head $(\mathrm{p}=0.11), \mathrm{n}=9$ rats, one-way ANOVA Tukey's corrected post-hoc comparison.

i, OTR-antagonist i.c.v. infusion decreases social interaction even in presence of pharmacological activation (hM3D-Gq) of parvOT neurons. Percentage of social interaction time in different conditions: saline (control), CNO, OTR-antagonist, or CNO + OTR-antagonist administration. Time spent social interacting over 5-minute sessions. Saline i.p. and i.c.v.: $90 \pm 19$ s, CNO i.p. and saline i.c.v.: $105 \pm 15 \mathrm{~s}, * \mathrm{p}=0.04, \mathrm{n}=6$ rats, saline i.p. and OTR-a i.c.v.: $54 \pm 17 \mathrm{~s}, * * \mathrm{p}=0.007$, CNO i.p. and OTR-a i.c.v.: $56 \pm 16 \mathrm{~s}, * * \mathrm{p}=0.009, \mathrm{n}=6$ rats, one-way ANOVA Tukey's corrected post-hoc comparison.

j, Control group in which parvOT neurons express GFP. Saline i.p. and i.c.v.: $88 \pm 18$ s, CNO i.p. and saline i.c.v.: $89 \pm 14 \mathrm{~s}, \mathrm{n}=5$ rats, saline i.p. and OTR-a i.c.v.: $53 \pm 14 \mathrm{~s}$, ${ }^{* *} \mathrm{p}=0.008$, CNO i.p. 
801

802

803

\section{METHODS}

805

806

807

808

809

810

811

812

813

814

815

816

817

818

820

821

822

823

824

\section{$\underline{\text { Animals }}$}

$\underline{\text { Viruses }}$

and OTR-a i.c.v.: $57 \pm 15 \mathrm{~s}, * * \mathrm{p}=0.001, \mathrm{n}=5$ rats, one-way ANOVA Tukey's corrected post-hoc comparison. All data represented as mean $\pm \mathrm{SEM}$.

Four to eight-week old female Wistar rats purchased from Janvier, France and were housed under standard laboratory conditions (12-h light/dark cycle, lights on at $07: 00,22-24{ }^{\circ} \mathrm{C}, 50 \pm 5 \%$ humidity, free access to food and water). All experiments were conducted under license G-102/17 (authorized by the German Animal Ethics Committee of the Baden Württemberg, Regierungspräsidium Karlsruhe) and in accordance with the German law, under license 36682016011815445431 from the French Ministry, and EU regulations. In total, 194 rats were used, of which 15 were excluded due to mistargeting or insufficient expression of viral vectors (Supplementary Table 3).

Recombinant adeno-associated viruses (rAAVs, serotype 1/2) used in this study (carrying conserved region of the oxytocin (OT) promoter and genes of interest in direct or "floxed" orientations) were cloned and produced as reported previously ${ }^{5,13,30,49}$. HEK293T cells (\#240073, Addgene, USA) were used for the virus production. rAAVs produced included: rAAV-OTpmCherry/Venus, rAAV-OTp-ChR2-mCherry, rAAV-OTp-DIO-ChR2-mCherry, rAAV-OTp-DIOhM3D(Gq)-mCherry, rAAV-OTp-DIO-hM4D(Gi)-mCherry, rAAV-OTp-DIO-GFP, rAAV-OTpDIO-ChR2-EYFP, rAAV-OTp-GCaMP6s, rAAV-OTp-TCB (TVA fused mCherry), rAAV-Ef1ADIO-oG. The canine adenovirus serotype 2 (CAV2-CMV-Cre) was purchased from the Institute of Molecular Genetics in Montpellier CNRS, France ${ }^{24}$. The retrograde AAV (rAAVretro-Ef1A-Cre) 
was purchased from the Salk Institute Viral Vector Core, CA, USA. Modified rabies virus was produced at the Gene Center rabies laboratory, Ludwig Maximillian University, Munich, Germany.

\section{$\underline{\text { Stereotactic injections of viral vectors }}$}

For stereotactic injections of viruses, rats were anesthetized with a mixture of ketamine $(65 \mathrm{mg} / \mathrm{kg}$ b.w.) and xylazine (14 mg/kg b.w.). rAAV genomic titers were determined with QuickTiter AAV Quantitation Kit (Cell Biolabs, Inc., San Diego, California, USA) and RT-PCR using the ABI 7700 cycler (Applied Biosystems, California, USA). rAAVs titers were between $10^{9}-10^{10}$ genomic copies/ $\mu 1$. We injected $300 \mathrm{nl}$ per PVN. CAV2-Cre was purchased from the Institute of Molecular Genetics, Montpellier (diluted to $10^{9}$ genomic copies/ $\mu 1,300 \mathrm{nl}$ per SON). Viruses were injected via a glass pipette into the target regions at $150 \mathrm{nl} / \mathrm{min}$ using a syringe pump as described $\mathrm{in}^{50}$. Coordinates were chosen in accordance to a rat brain atlas ${ }^{51}$ for PVN (A/P: $-1.8 \mathrm{~mm}, \mathrm{M} / \mathrm{L}: \pm 0.3$ $\mathrm{mm}, \mathrm{D} / \mathrm{V}:-8 \mathrm{~mm})$, for $\mathrm{SON}(\mathrm{A} / \mathrm{P}:-1.8 \mathrm{~mm}, \mathrm{M} / \mathrm{L}: \pm 1.2 \mathrm{~mm}, \mathrm{D} / \mathrm{V}-9.25 \mathrm{~mm})$, and for posterior pituitary (A/P: - $5.6 \mathrm{~mm}, \mathrm{M} / \mathrm{L}: \pm 0.1 \mathrm{~mm}, \mathrm{D} / \mathrm{V}:-10.5 \mathrm{~mm})$. Verification of injection and implantation sites and expression of genes of interest were confirmed in all rats post-hoc in $50-\mu \mathrm{m}$ sections containing the PVN and SON (see "Histology" section).

\section{$\underline{\text { Ex vivo experiments }}$}

\section{Slices preparation}

4-8 weeks after injection of viruses into the PVN and SON of 5 weeks old virgin female rats, animals were anesthetized using ketamine (Imalgene $90 \mathrm{mg} / \mathrm{kg}$ ) and xylazine (Rompun, $10 \mathrm{mg} / \mathrm{kg}$ ) administered intraperitoneally. Then, intracardiac perfusion were performed with an ice-cold NMDG based artificial cerebro spinal fluid (aCSF) was used containing (in mM): NMDG (93), KCl (2.5), $\mathrm{NaH}_{2} \mathrm{PO}_{4}$ (1.25), $\mathrm{NaHCO}_{3}(30), \mathrm{MgSO}_{4}(10), \mathrm{CaCl}_{2}$ (0.5), HEPES (20), D-Glucose (25), Lascorbic acid (5), Thiourea (2), Sodium pyruvate (3), N-acetyl-L-cysteine (10), Kynurenic acid (2). 
$\mathrm{pH}$ was adjusted to 7.4 using either $\mathrm{NaOH}$ or $\mathrm{HCl}$, this after bubbling in $95 \% \mathrm{O}_{2}-5 \% \mathrm{CO}_{2}$ gas. Rats

851 were then decapited, brains were removed, $350 \mu \mathrm{m}$ thick coronal slices containing the

852 hypothalamus was obtained using a Leica VT1000s vibratome. Slices were warmed 10 minutes in

$85335^{\circ} \mathrm{C}$ NMDG aCSF and placed for minimum 1 hour in a room tempered holding chamber,

854 containing normal aCSFs. Normal aCSF, also used during all ex vivo experiments, is composed of 855 (in mM): $\mathrm{NaCl}(124), \mathrm{KCl}(2.5), \mathrm{NaH}_{2} \mathrm{PO}_{4}(1.25), \mathrm{NaHCO}_{3}(26), \mathrm{MgSO}_{4}$ (2), $\mathrm{CaCl}_{2}$ (2), D-Glucose 856 (15), adjusted for $\mathrm{pH}$ values of 7.4 with $\mathrm{HCL}$ or $\mathrm{NaOH}$ and continuously bubbled in $95 \% \mathrm{O}_{2}-5 \%$ $857 \mathrm{CO}_{2}$ gas. All aCSFs were checked for osmolality and kept for values between 305-310 mOsm. In 858 electrophysiology or calcium imaging experiments, slices were transferred from the holding 859 chamber to an immersion recording chamber and superfused at a rate of $2 \mathrm{ml} / \mathrm{min}$. CNO containing 860 solution $(10 \mu \mathrm{M})$ were bath applied through a 6 min long pumping, corresponding to several times 861 the volume of the recording chamber (2 applications per slice maximum). All ex vivo experiments 862 were conducted at room temperature.

\section{Patch clamp recording}

865 Whole-cell patch-clamp recordings were visually guided by infrared oblique light videomicroscopy 866 (DM-LFS; Leica), using 4-9 $\mathrm{M} \Omega$ borosilicate pipettes filled with a $\mathrm{KMeSO}_{4}$ based intrapipette 867 solution composed of containing (in $\mathrm{mM}$ ): $\mathrm{KMeSO}_{4}$ (135), $\mathrm{NaCl}$ (8), HEPES (10), $\mathrm{ATPNa}_{2}$ (2), 868 GTPNa (0.3). The $\mathrm{pH}$ was adjusted to 7.3 with $\mathrm{KOH}$ and osmolality checked to be $300 \mathrm{mOsm} / 1$, 869 adjusted with sucrose if needed. Data were acquired with an Axopatch 200B (Axon Instruments) 870 amplifier and digitized with a Digidata 1440A (Molecular Devices, CA, USA). Series capacitances 871 and resistances were compensated electronically. Data sampled at $20 \mathrm{kHz}$ and lowpass filtered at 5 $872 \mathrm{kHz}$ using the pClamp10 software (Axon Instruments). Further analysis was performed using 873 Clampfit 10.7 (Molecular Devices; CA, USA) and Mini analysis 6 software (Synaptosoft, NJ, USA) 
874 in a semi-automated fashion (automatic detection of events with chosen parameters followed by a

875 visual validation).

876

877 Evoked activity. To test effects of CNO on neuronal excitability ex vivo, we used a current step 878 method. To this purpose, we make $\mathrm{PVN} \rightarrow \mathrm{SON}$ projecting neurons express the DREADD receptors 879 by injecting rats' SON with a CAV2-Cre virus (rAAV-CAV-Cre) and PVN with an OT specific 880 Cre-inducible DREADD construct (rAAV-OTp-DIO-hM4D(Gi)-mCherry or OTp-DIO-hM3D(Gq)881 mCherry). 6-8 weeks after infection, coronal slices were prepared and fluorescent neurons 882 (indicative of the viral expression) were selected for whole cell patch-clamp recordings. After 883 establishing the clamp, neurons were recorded in current clamp mode with 0pA injected. To test the 884 effect of the effects of DREADD activation - hM4D(Gi) or hM3D $(\mathrm{Gq})$ - neurons were subjected to 885 the following current steps. For hM4D(Gi), neurons received an injection of an $-100 \mathrm{pA}$ negative current to hyperpolarize the neuron membrane (reaching $-100 \mathrm{mV}$ ) before each step. These steps 887 start at $-80 \mathrm{pA}$ and increased by $20 \mathrm{pA}$, reaching $+120 \mathrm{pA}$. For hM3D(Gq), steps start at $-20 \mathrm{pA}$ and 888 increased by $10 \mathrm{pA}$, reaching $80 \mathrm{pA}$. To quantify the effects of DREADD activation, the number of 889 action potentials triggered by these steps were evaluated.

Spontaneous activity. To evaluate the effect of DREADD activation on neuronal activity, neurons 891 were also recorded 2 minutes before and after CNO exposure in voltage or current clamp mode. In 892 these cases, the frequency of post-synaptic current (PSC) or action potentials (AP) were quantified.

893 Identification of parvOT and magnOT. The identity of PVN's OT neurons was verified through 894 a current step protocol ${ }^{52}$, this method has been used in several other studies in order to allow 895 discrimination between parvocellular and the magnocellular neurons ${ }^{13,53-56}$. Neurons received an 896 injection of an $-100 \mathrm{pA}$ current to hyperpolarize the neuron membrane (reaching $-100 \mathrm{mV}$ ) before 897 each step. These steps start at $0 \mathrm{pA}$ and increased by $20 \mathrm{pA}$, reaching $+60 \mathrm{pA}$. To discriminate 
898 between parvOT and magnOT, we have measured the hyperpolarising notch and the $\mathrm{T}$ outward 899 rectification.

900 ChR2 Stimulation of SON's ParvOT neurons. In order to decipher the connexion between SON 901 parvOT neurons and PVN magnOT neurons, we have used an optogenetic strategy. First, we have 902 identified PVN OT neurons by injecting rats' PVN with an rAAV containing the coding sequence 903 of the fluorescent marker Venus under the control of the OT promoter (OTp-Venus). Then, we 904 aimed to specifically activate SON $\rightarrow$ PVN projection by using a combination of two rAAV : the 905 first one were injected in the SON and induce the expression of the Cre recombinase in SON 906 targeting neurons, and the second one were injected in the PVN to allow the expression of the ChR2 907 in OT neurons after a Cre-dependant recombination (OTp-DIO-ChR2-mCherry). 6-8 weeks after 908 infection, coronal slices containing the PVN were prepared and the Venus positive / mCherry 909 negative neurons were selected for whole cell patch-clamp recordings. This combination of 910 fluorescent markers allows us to select PVN OT neurons that are not directly targeting the SON.

911 Neurons were recorded 2 minutes in voltage clamp to establish the baseline frequency of 912 postsynaptic currents (PSC) and then, we performed an optogenetic stimulation of ChR2-expressing 913 OTergic neurons by applying light pulses $(10 \mathrm{~ms}$ at $30 \mathrm{~Hz}$ for $20 \mathrm{~s})$ using light source $\mathrm{X}$-Cite ${ }^{\circledR}$ 914 110LED from Excelitas Technologies through a GFP filter, controlled with a Clampex-driven TTL. 915 Neurons were also recorded during the ChR2 stimulation in order to observed that the neurons are 916 not expressing ChR2 itself. Finally, we continue to recorded 10 minutes after the stimulation to 917 observed the effect of the SON parvOT neurons stimulation on the PSC frequency of the recorded 918 neurons. PSC were detected using Mini analysis 6 software (Synaptosoft, NJ, USA).

\section{Calcium Imaging}

921 To test whether the chemogenetic activation of $\mathrm{PVN} \rightarrow \mathrm{SON}$ projecting OTergic neurons can modify 922 the intra PVN microcircuits activity, we have used an ex vivo calcium imaging approach. To this 
923 goal, rats' SON have been infected with a CAV2-Cre and the PVN were infected with a virus 924 allowing the expression of $\mathrm{hM} 3 \mathrm{D}(\mathrm{Gq})$ under the control of OT promoter after a Cre-dependant 925 recombination (OTp-DIO-hM3D(Gq)-mCherry). We also make PVN OT neuron express the 926 calcium indicator GCaMP6s using a third viral vector (rAAV-OTp-GCaMP6s). 6-8 weeks after 927 infection, coronal slices containing the PVN were prepared and neurons which were positive for 928 GCaMP but negative for mCherry were recorded. To perform this fluorescence microscopy, we 929 have used a Zeiss Axio examiner microscope with a 40x water immersion objective (numerical 930 aperture of 1.0), mounted with a X-Light Confocal unit - CRESTOPT spinning disk. Images were 931 acquired at $5 \mathrm{~Hz}$ with an optiMOS sCMOS camera (Qimaging, BC, Canada). Neurons within a 932 confocal plane were illuminated for $100 \mathrm{~ms}$ at $\lambda=475 \mathrm{~nm}$ using a Spectra 7 LUMENCOR. The 933 different hardware elements were synchronized through the MetaFluor software (Molecular 934 Devices, LLC, Ca, USA). Neurons calcium levels were measured in hand drawn region on interest 935 (ROI). In all recordings, the Fiji rolling ball algorithm was used to increase signal/noise ratio. 936 Recordings in which movements / drifts were visible were discarded.

937 Off-line data analysis was performed using a custom written python-based script.

938 First, a linear regression and a median filter was applied to each trace. Peaks were then detected 939 using the 'find_peaks' function of the SciPy library. More precisely, fluorescence variation was 940 identified as a calcium peak if its prominence exceeds two times the standard deviation and if the 941 maximum peak value surpasses 3 fluorescence units. ROI with zero calcium variations were 942 excluded from the analysis. The remaining ROI were considered as living neurons and the number 943 of peaks was quantified before and after the drug application. The AUC was estimated as the sum 944 of the local area of each peak to avoid biased AUC estimation due to baseline drift. All these data 945 were normalized according to the duration of the recording and neurons were labelled as 946 "responsive" when their AUC or their number of peaks were increased by at least $20 \%$ after drug 947 application. Because the time post-stimulation is longer than the baseline, the probability of 948 observing a spontaneous calcium peak is stronger post-stimulation. To avoid this bias, neurons with 
949

950

951

952

953

954

955

956

957

958

959

960

961

962

963

964

965

966

967

968

969

970

971

972

973

only one calcium peak during the whole recording were removed from responsive neurons. The response probability was calculated as the number of responsive neurons with a least 1 calcium event per time bin (30s) divide per the number of responsive neurons in each recording. Finally, all data were normalized per slice and this result was used as statistical unit. All data were compared using paired statistical analysis (before vs after drug application) and the results are expressed in ratio (baseline/drug effect), so a ratio of 1 meaning neither an increase or a decrease of the measured parameter.

\section{$\underline{\text { In vivo optoelectrode recordings }}$}

\section{Implantation of opto-electrodes}

Silicon probes (A1x32-Poly3-10mm, NeuroNexus) containing a 32-channel single shank combined with an optic fiber (diameter: $100 \mu \mathrm{m}$, Thorlabs) (opto-electrodes) were used in acute (anaesthetized and head-fixed) recordings. For freely moving recordings, thirty-two channel chronic optoelectrodes were hand-made, consisting of eight tetrodes and one specially designed microdrive. The microdrives and tetrodes were manually assembled as described previously ${ }^{57}$. The tetrodes were made with 0.0005-inch Tungsen wires (Stablohm 675, California Fine Wire Company, CA, USA). Eight tetrodes and an optic fiber (200 $\mu \mathrm{m}$, Thorlabs) were loaded into the micro-drive via a guiding tube and were arranged in parallel order. Assembled opto-electrodes were gold plated and impedance of each channel was measured between 250 and $350 \mathrm{kOhm}$. For implantation, rats were anesthetized with $2 \%$ isoflurane and placed in a stereotaxic frame. Bregma position and horizontal level were aligned during the implantation. Opto-electrode tips were implanted into the target location and the microdrive was fixed on the skull by six micro screws (Knupfer, Germany) and dental cement (Paladur, Heraeus Kulzer, Germany).

\section{Optogenetic identification of oxytocin neurons}


974 Electrophysiological signals were acquired by Open-Ephys acquisition board (Open Ephys, USA)

975 and sampled at $30 \mathrm{kHz}$. To identify ChR2-positive oxytocin neurons in the PVN, pulses of blue

976 light (wavelength $\lambda=473 \mathrm{~nm}$, DreamLasers) were delivered by the optic fiber while recording

977 extracellular electrical activity of the neurons. The pulse train was controlled by a pulse generator

978 (Master9, A.M.P.I.), pulses had a duration of $10 \mathrm{~ms}$ and were applied at stimulation frequencies of

9791,5 , and $10 \mathrm{~Hz}$. In each session, the laser output at the optic fiber terminal was measured as 20

$980 \mathrm{~mW} / \mathrm{mm}^{2}$. Neurons with a clear time-locked response to light pulses (spikes within 2-8 ms from

981 onset of pulses) were classified as oxytocin neurons (Extended Data Fig. 1e).

982

983 Analysis of spike waveforms

984 Spike sorting was done manually in Plexon Offline Sorter 4.0 (Plexon, Inc., TX, USA), with 985 tetrodes mode. The raw data were filtered at $250 \mathrm{~Hz}$ with a butterworth high-pass filter, and 986 waveform detection thresholds were placed at $-0.5-0.8 \%$ of $\mathrm{ADC}$ range (or $-0.32 \sim-0.51 \mathrm{mV}$ ), 987 depending on the signal-to-noise ratio. Magnocellular neurons have spikes with a width at half 988 amplitude of about $0.5 \mathrm{~ms}$, an absolute refractory period of about $2.5 \mathrm{~ms}$ and a long relative 989 refractory period reflecting a prominent hyperpolarizing afterpotential ${ }^{17}$. Therefore, the sample 990 length in waveform detection was set to $1.4 \mathrm{~ms}(400 \mu \mathrm{s}$ pre-threshold period, at the $30 \mathrm{kHz}$ 991 sampling rate, a single waveform consists of 42 data points, and in the tetrodes waveform, each unit 992 detected 168 data points), and dead time was set to $1.2 \mathrm{~ms}$. Next, the detected waveforms were 993 aligned at the valley point, when the neurons were depolarized at their maximum, and Principle 994 Component Analysis (PCA) and Slice features of waveform were plotted and projected into 3D 995 space for visual separation of clusters into presumptive single-units. The timestamp feature was 996 used to exclude mechanical noise recorded at same time across 4 channels among the tetrodes. In 997 different recording sessions (e.g., open field and social interaction), we analyzed whether the 998 features of spike waveforms remain consistent with the 3D plot results. After clustering, units with 999 a minimum inter-event interval exceeding $2500 \mu$ s were accepted as single hypothalamic neurons. 
1000 Units displaying minimum inter-event intervals between 1200-2500 $\mu$ s were recognized as arising

1001 from multiple neurons and were excluded from the statistics of the study.

1002

\section{Statistical analyses of spike patterning}

1004 From segments of stationary activity recorded in open field conditions, inter-spike interval 1005 distributions were constructed to verify that these were consistent with distributions characteristic 1006 of oxytocin neurons under basal conditions recorded in anesthetized rats ${ }^{58}$. To quantify the 1007 regularity of spike firing, we calculated the index of dispersion (IoD) of firing rate in 1-s bins as the 1008 ratio of the variance to the mean. For events that arise as a result of a random process that is 1009 invariant in time, the index will be equal to 1 independently of the mean rate, and independently of

1010 the binwidth. If events arise more regularly than chance, the index will be less than 1 , and if they 1011 are more variable than expected by chance - as when spikes occur in clusters or bursts, the index 1012 will be greater than 1.

1013 In oxytocin neurons, spikes cannot arise purely randomly because of the refractory period, and the 1014 IoD reduces slightly with increasing firing rate because at higher rates the relative refractory period 1015 is larger as a proportion of the mean interspike interval. The $\mathrm{IoD}$ also reduces with increasing 1016 binwidth because oxytocin neurons also display a prolonged activity-dependent 1017 afterhyperpolarization that acts to stabilize mean firing rates over a timescale of seconds. 1018 Collectively the known intrinsic membrane properties of rat oxytocin neurons, as tested through 1019 computational models, imply that if spikes arise as a result of a purely random and time-invariant 1020 process, then the $\mathrm{IoD}$ of firing rate in 1-s bins will be in the range $0.3-1$ for neurons firing at up to 6 1021 spikes/s, depending on firing rate and on individual variability in membrane properties ${ }^{17,58}$.

\section{Local field potentials in the PVN}

1024 Local field potentials (LFPs) were sampled at $1 \mathrm{kHz}$ with low-pass filter. Subsequent analysis was 1025 done using custom MATLAB (MathWorks, USA) scripts. We estimated power spectrum density 
1026 (PSD) of LFP signal using multi-tapper approach based on Thomson's method ('pmtm' function).

1027 Spectrograms were computed for each recording using standard 'spectrogram' function. Power of 1028 theta oscillations was calculated as average of PSD in the range 5-10 Hz. Phase-lock analysis was 1029 performed to investigate the relation between theta oscillations in the PVN and the timing of spikes

1030 in oxytocin neurons. The phase of the oscillatory activity was extracted with Hilbert transformation 1031 ('Hilbert' function) and converted into angle degrees. Then, we used Rayleigh's tests for circular 1032 uniformity, which indicates whether there is a significant correlation between the timing of spikes 1033 and a specific phase of the theta cycle (Extended Data Fig. 1h-j).

1034

\section{In vivo fiber photometry}

\section{Optic guided implantation of optic fibers}

1037 We injected a modified adenovirus (AAV-OTp-GCaMP6s) bilaterally into the PVN or SON to 1038 transduce expression of the $\mathrm{Ca}^{2+}$ indicator GCaMP6s in oxytocin neurons, and verified that this was 1039 expressed cell-specifically $(87 \pm 4 \%$ of oxytocin neurons, $n=1371$ neurons, $n=4$ rats, Fig. 4 p).

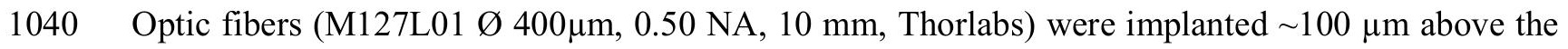
1041 dorsal border of the PVN (A/P: $-1.8 \mathrm{~mm}, \mathrm{M} / \mathrm{L}: 0.35 \mathrm{~mm}, \mathrm{D} / \mathrm{V}:-7.85 \mathrm{~mm}$ ) or SON (A/P: $-1.25 \mathrm{~mm}$,

$1042 \mathrm{M} / \mathrm{L}: 1.90 \mathrm{~mm}, \mathrm{D} / \mathrm{V}:-9.0 \mathrm{~mm}$ ) under 1.5\% isoflurane anesthesia. Four $1 \mathrm{~mm}$ screws (Knupfer,

1043 Germany) and a metal implant guide (OGL, Thorlabs) were attached to skull with OptiBond FL

1044 (Kerr, Germany) and fixed by dental cement (Paladur, Heraeus Kulzer, Germany).

1045 During implantation, the implantable cannula was fixed in an adaptor (ADAL3, Thorlabs) attached 1046 to stereotactic holder; while the other end of the cannula was connected through a pre-bleached 1047 Patch cord (FP400URT, Thorlabs) to the photodetector and LED of fiber photometry system (FOM, 1048 NPI Electronic, Germany). The digitalized photometry signal was monitored and recorded via 1049 digital input/output (DIO) board (OpenEphys, USA) to the DAQ system. (OpenEphys, USA) with $1050 \quad 0.1-20 \mathrm{~Hz}$ bandpass filter and $20 \mathrm{~s}$ time scale set in to visualize the $\mathrm{Ca}^{2+}$ signal online, while the 1051 cannula tip was gradually lowered into the PVN at $1 \mathrm{~mm} / \mathrm{min}$. When the optic fiber tip was close to 
1052 the PVN where GCaMP6s was expressed, a slight increase in the signal baseline and a minor

1053 spontaneous fluctuation could be visually detected. During implantation, rats were under $1.5 \%$

1054 isoflurane anesthesia and body temperature was kept stable at $37^{\circ} \mathrm{C}$ by a heating plate (RWD,

1055 China). The LED power in fiber photometry system was set at a constant value between 5-10

$1056 \mathrm{~mW} / \mathrm{mm}^{2}$. The fiber photometry recordings were conducted after one week of recovery from the

1057 implantation. Fiber photometry raw data were sampled at $30 \mathrm{kHz}$ in OpenEphys GUI and analyzed

1058 with custom written MATLAB scripts.

1059

\section{Fiber photometry data analysis}

1061 Digitalized optical signal acquired from the fiber photometry system was first downsampled at 3000

$1062 \mathrm{~Hz}$ and then low-pass filtered (MATLAB 'butteworth' function) at $10 \mathrm{~Hz}$ to exclude noise at higher

1063 frequency. Secondly, to correct the baseline drifting due to photo-bleaching of fluorophores we

1064 fitted the signal with a polynomial curve (MATLAB 'polyfit' function) and subtracted it to the

1065 signal. Next, we smoothed the signal with a Savitzky-Golay filter (MATLAB 'smooth' function,

1066 option 'sgolay'). For each experiment, the signal $\mathrm{F}$ was converted to $\Delta \mathrm{F} / \mathrm{F}_{0}$ by

$$
\Delta F / F(t)=\frac{F(t)-F_{0}}{F_{0}}
$$

where $\mathrm{F}_{0}$ was calculated as the average value of $\mathrm{F}$ of a 600 second recording at the beginning of the experiment. The data was subdivided in 1 min bins and the mean $\Delta \mathrm{F} / \mathrm{F}_{0}$ was calculated for each bin.

We detected calcium transients similar to those reported by our previous study ${ }^{59}$. Finally, we 1070 calculated the area under the curve (AUC) of the $\mathrm{Ca}^{2+}$ signal (MATLAB 'trapz' function) to 1071 estimate the cumulative fluorescence for each bin and normalized the AUC to values from 0 to 1.

1072 Values of normalized AUC was displayed in $1 \mathrm{~min}$ bin and averaged in $30 \mathrm{~min}$ bins. The ratios of 1073 AUCs between experimental and control conditions were used for quantitative analysis and called 1074 "relative AUC increase". 


\section{Application of airpuffs and OT neurons response}

1077 Airpuffs from a pressured air can (Toolcraft 20793T $400 \mathrm{ml}$, Germany) were applied through a stiff 1078 micropipette tip with a 2-mm opening positioned $10-15 \mathrm{~mm}$ above the skin in the area of $\sim 2 \mathrm{~cm}^{2}$. 1079 A plastic cover with 2-cm holes was placed above the rat's body to restrict the area of stimulation.

1080 The controlled air pressure was $1.139 \mathrm{~g} / \mathrm{cm}^{3}$. During in vivo electrophysiology recordings, in each 1081 stimulation point, 5 airpuffs (duration $0.2 \mathrm{~s}$, interval between puffs $1 \mathrm{~s}$ ) were delivered in sequence 1082 with intervals of 1 min between sequences (Fig. 2a and Extended Data Fig. 4). During fiber 1083 photometry recordings, one airpuff (duration $1 \mathrm{~s}$ ) was applied every 1 min (Fig. $\mathbf{2 e - j}$ ).

1084

1085

\section{OT neurons response to airpuff stimulations}

1086

We applied airpuffs to the skin of three regions of the rat's dorsal body area (anterior, central, and posterior part), two regions of the rat's ventral area (abdomen and anogenital area), and to the whiskers on both sides. We considered a recorded neuron as responsive to airpuff stimulations if the average firing rate after (from 0 to 2 s) stimuli onset increased of at least 2 times the standard deviation (SD) of the baseline activity ( $2 \mathrm{~s}$ prior to stimuli onset). Onset of the response was calculated as the time at which the firing rate of a responsive neurons increased of 1 time the SD of the baseline activity. We recorded the activity of $n=23$ oxytocin neurons in response to airpuffs applied on the rat's dorsal body area which showed variable response latencies up to $30 \mathrm{~s}$ (Extended Data Fig. 4a-b); 10 of those neurons exhibited a response within $1 \mathrm{~s}$ after stimuli onset and are shown in Fig. 2a-b.

\section{Blood sampling and plasma OT measurements}

To monitor neurohypophysial OT release after chemogenetic activation of hypothalamic parvOT neurons, we performed blood sampling from the jugular vein in urethane-anesthetized rats. After

1100 surgery, rats were placed on a heating pad for the rest of the experiment to maintain constant body 1101 temperature. The jugular vein catheter was connected to a 1-ml syringe containing sterile 
1102 heparinized saline (30 IU/ml). $45 \mathrm{~min}$ before, and $45 \mathrm{~min}$ as well as $90 \mathrm{~min}$ after ip CNO, $500 \mu \mathrm{l}$ of

1103 blood was drawn (Fig. 4q-r), which was replaced by $500 \mu$ l sterile saline. After each sample, the

1104 catheter was filled with heparinized saline to avoid blood clotting. Blood samples were collected in

1105 EDTA-tubes (Bayer, Germany) on ice, centrifuged $\left(5000 \mathrm{x} \mathrm{g}, 10 \mathrm{~min}, 4{ }^{\circ} \mathrm{C}\right)$, and $200-\mu 1$ plasma

1106 samples were stored at $-80^{\circ} \mathrm{C}$ prior to extraction and OT quantification by radioimmunoassay. OT

1107 content in extracted plasma was analyzed by a highly sensitive radioimmunoassay with a detection

1108 limit of $0.1 \mathrm{pg}$ and cross-reactivity $<0.7 \%$ (RIAgnosis, Germany) ${ }^{60,61}$.

1109

\section{Behavior}

1111 Starting from 14 days prior to behavioral tests, vaginal smears were collected to monitor ovarian

1112 cycle. Rats in metestrus, proestrus and estrus phases were excluded from experiments and

1113 reintroduced once they reached diestrus.

1114 Behavioral tests were conducted in an arena (material non-absorbent to odors) with dimensions

1115 60x60x60 cm under dim light condition (<20 lux; lux-meter SO 200K, Sauter, Germany). On the

1116 day before the test, the experimental rat was exposed to the arena for $15 \mathrm{~min}$ for habituation. The

1117 arena was cleaned with $70 \%$ ethanol after each session to eliminate residual odors. Experimental

1118 and stimuli rats were housed in separate cages and had not previous encountered each other before

1119 the social interaction tests. The same rat was exposed to social interaction tests twice on separate

1120 days, each time with a different social stimulus rat so that the experimental paradigm always

1121 represented interaction with a novel, unfamiliar conspecific.

\section{Open field test:}

1123 The experimental rat was placed in a corner of the arena and was allowed to freely explore the

1124 environment. These tests served as a "baseline" for social interaction tests.

\section{Free social interaction (FSI) test:}

1126 The experimental and the stimulus rats were placed in opposite corners of the arena at the same

1127 time and were allowed to freely interact with each other and/or explore the environment. 


\section{Chambered social interaction (CSI) test:}

1129 For this test, two plexiglas transparent meshes (dimensions 20x30x1 cm) provided with three 1130 opening/holes (dimensions $15 \times 0.75 \mathrm{~cm}$ ) were placed in two opposite corners of the arena. The 1131 mesh separated a little triangular area $(14 \times 14 \times 20 \mathrm{~cm}$, corresponding to $\sim 3 \%$ of the total area of

1132 the arena) to the rest of the arena (central compartment). The experimental rat was placed in the 1133 central compartment while the stimulus rat was placed in one of the two little compartments. The 1134 two rats were able to see, hear, and smell each other through the openings, but they were not able to 1135 touch one another.

\section{Chemogenetic inhibition or activation of parvocellular oxytocin neurons by DREADD}

1137 To selectively activate or inhibit parvocellular oxytocin neurons, rats were injected with rAAVOTp-DIO-hM3D(Gq)-mCherry (Parvo-Gq group), rAAV-OTp-DIO-hM4D(Gi)-mCherry (Parvo-Gi

1139 group), or rAAV-OTp-DIO-GFP (Parvo-GFP control group) into the PVN and CAV2-Cre into the 1140 SON, as previously described ${ }^{13}$.

1141 All groups (Parvo-Gq, Parvo-Gi, and Parvo-GFP) were subjected to the same protocol. On day 1

1142 experimental rats were exposed to the open field arena for $15 \mathrm{~min}$ for habituation. On day 2, the 1143 experimental rat was injected i.p. with either $\mathrm{CNO}$ or saline solution 60 min before beginning the 1144 tests and then was subjected to one CSI and one FSI session for 5 min each.

\section{Intracerebroventricular administration of OT-receptor antagonist}

1147 Guide cannulas were implanted above the lateral ventricle for intracerebroventricular (i.c.v.) 1148 infusion of OTR antagonist (OTR-a) des-Gly-NH $2, \mathrm{~d}\left(\mathrm{CH}_{2}\right)_{5}\left[\mathrm{Tyr}(\mathrm{Me})^{2}, \mathrm{Thr}^{4}\right] \mathrm{OVT}^{29}$. OTR-a 0.75

$1149 \mu \mathrm{g} / 5 \mu \mathrm{l}^{30,62}$ was infused 15 minutes prior to behavioral tests. Four groups of rats were studied, 1150 which received i.p. injection and i.c.v. infusion of Saline/Saline, CNO/Saline, Saline/OTR-a, or 1151 CNO/OTR-a respectively. 
1154 The videos were recorded using a GigE color HD camera (Basler AG, Germany). The tracks of the

1155 experimental and stimulus rat were extracted from videos using two softwares: Ethovision XT 11.5

1156 (Noldus) and MATLAB Toolbox idTracker (MathWorks). Results of the two softwares were

1157 compared and cross-validated. The distance moved by each rat, velocity, the time spent in different

1158 areas of the arena, and the distance between rats and time spent in close proximity were calculated

1159 automatically. Social interactions were also analyzed manually to classify social behaviors into

1160 different categories: 'sniffing', 'chasing', 'crawling on top', 'being crawled', and 'head-to-head'

1161 approaching; time spent by experimental rat for each behavioral category was used for all analysis.

1162 Manual scoring of social behavior scoring were done by a researcher (different from the one who

1163 performed the experiment) that was blind to treatment conditions.

1164 Ultrasonic vocalizations were recorded with an ultrasound microphone (Avisoft-Bioacustic,

1165 Germany) and analyzed with Avisoft-SASlab Pro 5.2 software. After calculation of sound

1166 spectrogram, vocalizations time, duration, and frequency were extracted. Each 'call' was classified

1167 into non-social (peak frequency $\sim 22 \mathrm{kHz}$ ) or appetitive/social (peak frequency $\sim 50 \mathrm{kHz}$ ) call.

1168 Social vocalizations were further classified in trills $(<10 \mathrm{~ms})$, single component calls $(>10 \mathrm{~ms}$, not

1169 modulated), and complex vocalizations ( $>10 \mathrm{~ms}$, frequency modulated or combined $)^{63}$.

1170

1171 Freely moving single unit recordings - experimental groups

1172 Open field and FSI group: experimental rats implanted with opto-electrodes for single unit

1173 recordings in the PVN were subjected to one open field session and one FSI session for $10 \mathrm{~min}$

1174 each. Between the two session the rat was place in the home cage (single-housed) for $15 \mathrm{~min}$.

1175 Open field, CSI, and FSI group: experimental rats implanted with opto-electrodes for single unit

1176 recordings in the PVN were subjected to one open field, one CSI, and one FSI session for 10 min

1177 each, without pauses in between. Stimulus rats were placed in one of the little chambers separated

1178 by a plexiglas mesh at the beginning of the CSI session; the wall was then lifted up (Fig. 6b) at the 
1179 beginning of the FSI session allowing the stimulus rat to join the experimental rat in the central 1180 compartment.

\section{Histology}

1183 Anesthetized rats were transcardially perfused with PBS followed by 4\% PFA. Brains were 1184 dissected out and post-fixed overnight in $4 \%$ PFA at $4{ }^{\circ} \mathrm{C}$ with gentle agitation. $50-\mu \mathrm{m}$ vibratome 1185 coronal sections containing the PVN and the SON were cut and collected. Immunohistochemistry 1186 was performed on free-floating sections with the following antibodies: anti-OT (PS38, 1:2000; 1187 mouse; kindly provided by Harold Gainer), anti-OT (T-5021, 1:50,000, Peninsula, guinea-pig), anti1188 synaptophysin (ab32127, 1:1,000, abcam anti-rabbit), anti-Ds-Red (\#632397, 1:1000; rabbit;

1189 Clontech), anti-GFP (ab13970, 1:1000, chicken, Abcam), anti c-fos (\#9F6, 1:500, rabbit, Cell 1190 Signaling), anti-Fluorogold (NM-101, 1:1000, guinea pig, Protos Biotech), anti-Cre (\#69050, 1191 1:2000, mouse, Novagen). Further information on validation of primary antibodies can be found in 1192 the Life Science Reporting Summary. The signals were visualized with the following secondary 1193 antibodies, CY3-conjugated (711-165-152) or CY5-conjugate (115-175-146, Jackson Immuno1194 Research Laboratories) or Alexa 488 (A11039) and 594 (A11012, Invitrogen) and Alexa-594 (7151195 585-151) and Alexa-647 (713-645-147, Jackson Immuno-Research Laboratories). All secondary 1196 antibodies were diluted 1:500.

\section{Fluorogold treatment and visualization}

1199 To discriminate between magno- and parvocellular oxytocin neurons, rats received a single 1200 injection of Fluorogold (Santa Cruz Biotechnology, Dallas, $15 \mathrm{mg} / \mathrm{kg}$ bw i.p.) 7 days before the 1201 perfusion. Brain sections were stained with a primary antibody for Fluorogold (guinea pig anti-FG, 1202 dilution 1:1000, Protos Biotech Corp, New York) and Fluorogold immunosignal was visualized by 1203 secondary antibodies conjugated with CY3 (Goat anti-rabbit, dilution 1:500, Jackson Immuno- 
1204 Research, Newmarket Suffolk, UK). The colocalization of Fluorogold, oxytocin, and c-fos signals

1205 were manually quantified in the PVN ( $\mathrm{n}=4$ rats; 6 sections/brain).

1206

\section{Images of immunostained tissue sections}

1208 All images were acquired on a Leica TCS SP5 (DKFZ Light Microscopy Facility) confocal laser1209 scanning microscope. Digitized images were analyzed using Fiji (NIMH, Bethesda, MD, USA) and 1210 Adobe Photoshop CS5 (Adobe, Mountain View, CA).

1211

\section{Confocal microscopy and 3D IMARIS analysis}

1213 For the 3D reconstruction of OT neurons, we took Z-stack images (50 $\mu \mathrm{m}$ depth, $1 \mu \mathrm{m}$ steps, $40 \mathrm{x}$

1214 magnification) of PVN and SON using a Zeiss LSM 780 confocal microscope (1024x1024 pixel,

1215 16-bit depth, pixel size 0.63-micron, zoom 0.7). Raw czi files were used for further analysis using

1216 IMARIS $^{26,27,64}$ software (Version 9.31, Oxford Instruments: https://imaris.oxinst.com). First,

1217 IMARIS was used to reconstruct the cellular surface using the following custom settings: surfaces

1218 Detail $0.700 \mu \mathrm{m}$ (smooth); thresholding Background subtraction (Local Contrast), diameter of

1219 largest Sphere, which fits into the object: 2.00; Color: base, diffusion transparency: 65\%. After

1220 surface reconstruction, we used the filter function to remove unspecific background signals: Filter:

1221 Volume $\max -400 \mu \mathrm{m}^{3}$. After deletion of all background signals the 'mask all' function was used

1222 to create the final surface reconstruction. Next, the surface reconstruction was used as the template

1223 for the filament reconstruction using the following custom settings: detect new starting points:

1224 largest Diameter $7.00 \mu \mathrm{m}$, seed points $0.300 \mu \mathrm{m}$; remove seed points around starting points:

1225 diameter of sphere regions: $15 \mu \mathrm{m}$. Seed points were corrected for (either placed in or removed

1226 from the center of the somata) manually if the IMARIS algorithm placed them incorrectly. All

1227 surface and filament parameters were exported into separate Excel files and used for data analysis.

1228 For all quantifications, we used 6-8 40x z-stacks per animal (2 z-stacks per brain hemisphere). We

1229 used a computer suited for IMARIS analysis (Intel Core i7 $8700 @ 3.2$ GHz, 64 GB RAM, x-64-bit, 
1230 Windows 10 Enterprise). All images used for analysis were taken with the same confocal settings

1231 (pinhole, laser intensity, digital gain and digital offset). Sholl analysis was performed using

1232 IMARIS in the filament reconstruction mode and individual data sets were exported into separate

1233 Excel files for further analysis. To assess the number of SYN+/GFP-positive axons, we used a

1234 simplified version of the Sholl analysis, where we only included the first 2-8 spheres (starting in the

1235 soma center) either until we could detect SYN+/GFP intersections or were more than $2 \mu \mathrm{m}$ apart

1236 from the border of the respective soma. The total amount of immunoflourescene (synaptophysin)

1237 was calculated using the extract intensity/number of spots function. First, we created spheres that

1238 precisely engulfed the respective somata (parvOT and magnOT neurons) so that both ends of the

1239 cell soma (maximum diameter) touched the border of the respective sphere. To account for

1240 individual variability in roundness and surface area, we calculated surface area for each individual

1241 OT cell using the surface reconstruction mode. Given that cells with a larger surface area occupy

1242 more three-dimensional space within the artificially constructed sphere that could confound precise

1243 quantification of SYN fluorescence, we adjusted each calculated value (SYN+ voxels per sphere)

1244 based on the surface area. Assuming an inverse near-linear relationship between cell volume and

1245 the total amount of SYN fluorescence within a sphere, we calculated the degree of occupancy (i.e.

1246 percentage) for each somata within the respective sphere. Finally, we calculated the final SYN+

1247 voxels using the following equation: (number of SYN+ voxels) * (degree of occupancy). For the

1248 quantification along the dendrites we used spheres with a $10 \mu \mathrm{m}$ radius along the dendrite for both

1249 parvOT and magnOT neurons.

1250

\section{Projection-specific trans-synaptic retrograde tracing}

1252 Input tracing experiments were performed in female Wistar rats (aged 10-12 weeks). We used

1253 EnvA-pseudotyped rabies virus $G$ deletion-mutant EnvA $\Delta \mathrm{G}-\mathrm{EGFP}\left(\mathrm{Rb}-\mathrm{GFP}{ }^{28}\right)$ to

1254 monosynaptically retrogradely trace neurons projecting to parvOT and magnOT neurons. Rb-GFP

1255 selectively enters neurons expressing the avian sarcoma and leucosis virus receptor (TVA), and can 
1256 only spread presynaptically from neurons expressing the rabies virus glycoprotein (we used the 1257 optimized glycoprotein, $\mathrm{oG}$, from ${ }^{65}$ ). We injected a $300 \mathrm{~nL}$ mixture of 1:1 rAAV-OTp-TCB:rAAV1258 Ef1A-DIO-oG into the right PVN of female rats. Then, to specifically trace inputs to parvOT 1259 neurons, we injected rats $(n=5)$ with CAV2-CMV-Cre into the right SON (Extended Data Fig. 8a). 1260 In another group of rats $(n=5)$, we employed a similar strategy to express oG only in magnOT 1261 neurons: we injected an AAV retrograde expressing Cre (rAAVretro-Ef1A-Cre) into the posterior 1262 pituitary (Extended Data Fig. 8c, based on ${ }^{66}$ ). This strategy makes Rb-GFP selectively enter in all 1263 OT neurons, but specifically spread retrogradely from neurons expressing oG (i.e, parvOT or 1264 magnOT neurons). After two weeks, we injected $300 \mathrm{~nL}$ of EnvA $\Delta \mathrm{G}$-EGFP into the right PVN, 1265 and seven days later, animals were perfused with 4\% PFA. The number of projecting neurons was 1266 quantified from brain sections as follows: every third $50-\mu \mathrm{m}$ section was imaged and neurons were 1267 counted, and then multiplied by three, to estimate the real number of inputs. GFP+ neurons on the 1268 injected hemisphere were counted and assigned to brain areas based on classifications of the 1269 Paxinos Mouse Brain Atlas37, using anatomical landmarks in the sections visualized by tissue 1270 autofluorescence. Very few contralateral inputs were noticed and we thus decided to neglect them.

1271 While we had good infection at injection sites for both parvOT and magnOT groups (Extended

1272 Data Fig. 8g), starter neurons could not be reliably counted, as rabies virus toxicity prevented us to 1273 correctly visualize mCherry in the PVN. Thus, the analysis presented here does not take into 1274 account inputs to OT neurons from within PVN. The percentage of inputs from each region was 1275 obtained by dividing the number of inputs from one region per the total number of inputs. Input 1276 regions that were detected in a subset of animals only were discarded from analysis. We used 1277 unpaired two-sided Student's t tests to compare the total number of inputs to parvOT and magnOT 1278 neurons and Chi squared tests to compare proportions of inputs between regions.

1279 We controlled that TVA was selectively expressed in OT neurons by injecting control rats $(\mathrm{n}=2)$ 1280 with rAAV-OTp-TCB in the PVN, and staining for OT. This revealed that most OT neurons 1281 expressed mCherry and that no non-OT neurons expressed mCherry (Extended Data Fig. 8e). 
1282 Furthermore, we verified that Rb-GFP was selectively entering OT neurons by injecting control rats

1283 (n=2) with rAAV-OTp-TCB, and Rb-GFP 2 weeks later. This resulted in specific expression of

1284 GFP in PVN OT neurons (Extended Data Fig. 8f).

1285 In each rat, we confirmed SON injection site by staining for Cre for the parvOT neurons tracing

1286 (Extended Data Fig. 8a,b) and by injecting a virus Cre-dependently expressing mCherry in the

1287 SON of magnOT neurons tracing, which led to expression of mCherry in SON magnocellular

1288 neurons (Extended Data Fig. 8c,d).

1289

1290 Oxytocin secretion model

1291 The OT secretion mode ${ }^{17}$ simulates stimulus-secretion coupling in oxytocin neurons. The model is

1292 a continuous approximation of the stochastic release process from all neuronal compartments. It is

1293 based on extensive studies on activity-dependent hormone secretion from magnocellular

1294 neurosecretory neurons ${ }^{58}$ and it matches experimental data closely. In the model, when spikes

1295 invade the secretory terminals, exocytosis occurs in response to fast rising $\mathrm{Ca}^{2+}$ concentrations $(e)$.

1296 At higher frequencies, the spikes broaden, producing a larger increase of $e$. The rate of secretion is

1297 modeled as the product of: $e$ raised to the power of $\varphi$ (which accounts for the cooperativeness of the

$1298 \mathrm{Ca}^{2+}$ activation), of the pool of releasable OT $p$, and a secretion scaling factor $\alpha$, and is calculated 1299 as:

$$
s=e^{\varphi} \cdot \alpha \cdot p
$$

1301 where $\varphi=2, \alpha=0.003 \mathrm{pg} / \mathrm{s}$.

1302 The non-linear dependence of the secretion rate gives high secretion probability upon short spike

1303 intervals. To infer OT secretion arising from the spike trains observed in the present study, the

1304 recorded event timings were used to drive the secretion model described fully elsewhere ${ }^{17}$. The

1305 published model is scaled to quantitatively match secretion from the pituitary nerve terminals of a

1306 single oxytocin neuron. The scaling factor $\alpha$ cannot be used for absolute quantitative estimates of 
1307 release within the brain, but the relative efficacy of two firing patterns can be compared using the

1308 model, as $\alpha$ is eliminated in the ratio.

\section{$\underline{\text { Statistics }}$}

1311 Statistical analyses were performed using SigmaPlot 11 (Systat, USA) and GraphPad Prism 7.05

1312 (GraphPad Software, San Diego, California, USA). Two-sided Wilcoxon signed-rank W test was 1313 used to compare the variation of spike frequencies measured for the same neuron in different 1314 conditions. Two-sided Mann-Whitney U test was used to compare low threshold depolarization in 1315 different cells. Two-sided Student's t tests were used to compare average values in two conditions 1316 when the data satisfied assumptions of normality. One-way ANOVA, followed by multiple

1317 comparison post-hoc test, was used to compare averages in three or more conditions. Two-way 1318 ANOVA, followed by multiple comparison post-hoc test, was used to analyze electrophysiological 1319 or behavioral data with repeated measures and CNO/Saline/OTR-a treatment (time $\mathrm{x}$ treatment). No 1320 statistical methods were used to pre-determine sample size but our sample sizes are similar to those 1321 reported in previous publications ${ }^{5,13,30}$.

1322 Differences were considered significant for $\mathrm{p}<0.05$. Asterisks were used to indicate the

1323 significance level: $* 0.01 \leq \mathrm{p}<0.05, * * 0.001 \leq \mathrm{p}<0.01, * * * \mathrm{p}<0.001$. Statistical analyses of 1324 neuronal spike trains and local field potentials, such as peristimulus time histograms (PSTHs), auto1325 and cross-correlation, spikes burst analysis, power spectrum density, and phase-locking were 1326 performed using NeuroExplorer 3 (Nex Technologies, Colorado, USA) and custom written Matlab 1327 scripts.

\section{$\underline{\text { Data and code availability }}$}

1330 Python code (used for ex-vivo calcium imaging data analysis in Fig. 4a-d) and Matlab code (used 1331 for in-vivo fiber photometry data analysis in Fig. 4e-o, Extended Data Fig. 7a-n) can be found in 1332 Supplementary Software. All data that supporting the findings of this study, as well as Matlab codes 
1333 for the analysis of extracellular recording data, are available from the corresponding author upon

1334 reasonable request.

1335

\section{Methods only references}

1337 49. Menon, R. et al. Oxytocin signaling in the lateral septum prevents social fear during 1338 lactation. Curr. Biol. 28, 1066-1078.e6 (2018).

1339 50. Grinevich, V. et al. Somatic transgenesis (Viral Vectors). 3, 243-274 (2016).

1340 51. Paxinos, G. \& Watson, C. The Rat Brain in Stereotaxic Coordinates Seventh Edition. $1341 \quad$ Elsevier Acad. Press (2014).

1342 52. Tasker, J. G. \& Dudek, F. E. Electrophysiological properties of neurones in the region of the 1343 paraventricular nucleus in slices of rat hypothalamus. J. Physiol. 434, 271-293 (1991).

1344 53. Chu, C.-P. et al. Effects of Stresscopin on Rat Hypothalamic Paraventricular Nucleus $1345 \quad$ Neurons In Vitro. PLoS One 8, e53863 (2013).

1346 54. Luther, J. A. \& Tasker, J. G. Voltage-gated currents distinguish parvocellular from 1347 magnocellular neurones in the rat hypothalamic paraventricular nucleus. J. Physiol. 523, 1348 193-209 (2000).

1349 55. Luther, J. A. et al. Neurosecretory and Non-Neurosecretory Parvocellular Neurones of the 1350 Hypothalamic Paraventricular Nucleus Express Distinct Electrophysiological Properties. J. $1351 \quad$ Neuroendocrinol. 14, 929-932 (2002).

1352 56. Yuill, E. A., Hoyda, T. D., Ferri, C. C., Zhou, Q.-Y. \& Ferguson, A. V. Prokineticin 2 1353 depolarizes paraventricular nucleus magnocellular and parvocellular neurons. Eur. J. $1354 \quad$ Neurosci. 25, 425-434 (2007).

1355 57. Tang, Y., Benusiglio, D., Grinevich, V. \& Lin, L. Distinct types of feeding related neurons in 1356 mouse hypothalamus. Front. Behav. Neurosci. 10, 91 (2016).

1357 58. Maícas Royo, J., Brown, C. H., Leng, G. \& MacGregor, D. J. Oxytocin neurones: intrinsic 1358 mechanisms governing the regularity of spiking activity. J. Neuroendocrinol. 28, (2016). 
1359 59. Grund, T. et al. Chemogenetic activation of oxytocin neurons: Temporal dynamics, 1360 hormonal release, and behavioral consequences. Psychoneuroendocrinology 106, 77-84 $1361 \quad$ (2019).

1362 60. Jong, T. R. de et al. Salivary oxytocin concentrations in response to running, sexual self1363 stimulation, breastfeeding and the TSST: The Regensburg Oxytocin Challenge (ROC) study. $1364 \quad$ Psychoneuroendocrinology 62, 381-388 (2015).

1365 61. Landgraf, R., Neumann, I., Holsboer, F. \& Pittman, Q. J. Interleukin-1 $\beta$ stimulates both 1366 central and peripheral release of vasopressin and oxytocin in the rat. Eur. J. Neurosci. 7, $1367 \quad 592-598(1995)$.

1368 62. Neumann, I. D., Maloumby, R., Beiderbeck, D. I., Lukas, M. \& Landgraf, R. Increased brain 1369 and plasma oxytocin after nasal and peripheral administration in rats and mice. $1370 \quad$ Psychoneuroendocrinology 38, 1985-1993 (2013).

1371 63. Ishiyama, S. \& Brecht, M. Neural correlates of ticklishness in the rat somatosensory cortex. $1372 \quad$ Science 354, 757-760 (2016).

1373 64. Althammer, F., Ferreira-Neto, H. C., Rubaharan, M., Roy, K. R. \& Stern, J. E. Three1374 dimensional morphometric analysis reveals time-dependent structural changes in microglia and astrocytes in the central amygdala and hypothalamic paraventricular nucleus of heart failure rats. Research square, preprint (2020). doi:10.21203/rs.3.rs-22630/v1

65. Kim, E. J., Jacobs, M. W., Ito-Cole, T. \& Callaway, E. M. Improved monosynaptic neural circuit tracing using engineered rabies virus glycoproteins. Cell Rep. 15, 692-699 (2016).

66. Bin Zhang, Liyao Qiu, Wei Xiao, Hong Ni, Lunhao Chen, Fan Wang, Weihao Mai, Hui 1380 Gong, Shumin Duan, Anan Li, V. O. P. G. Reconstruction of the hypothalamoneurohypophysial system and functional dissection of magnocellular oxytocin neurons in the brain. bioRxiv, preprint (2020). doi:10.1101/2020.03.26.007070 
Figure 1

a
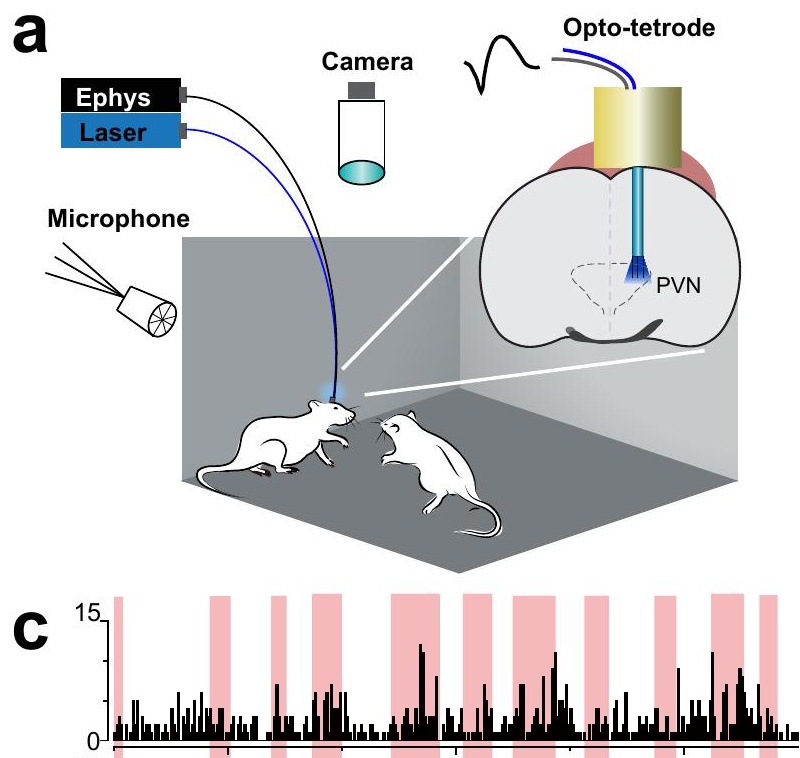

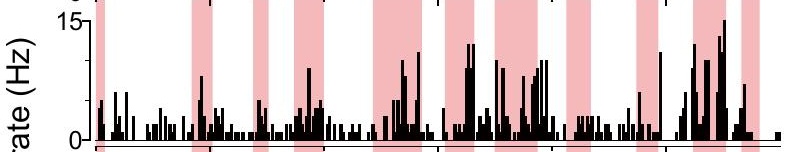

:

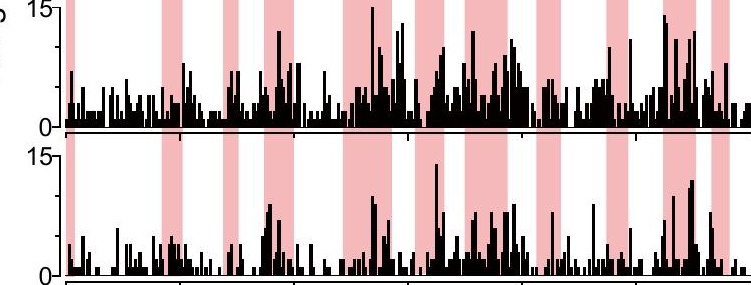

e

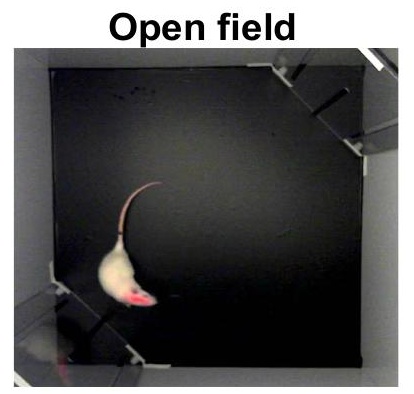

产 Average firing rate $1.0 \mathrm{~Hz}$ क b

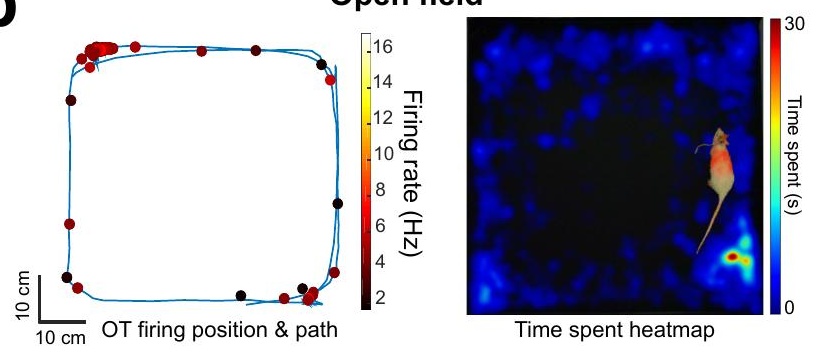

Social interaction

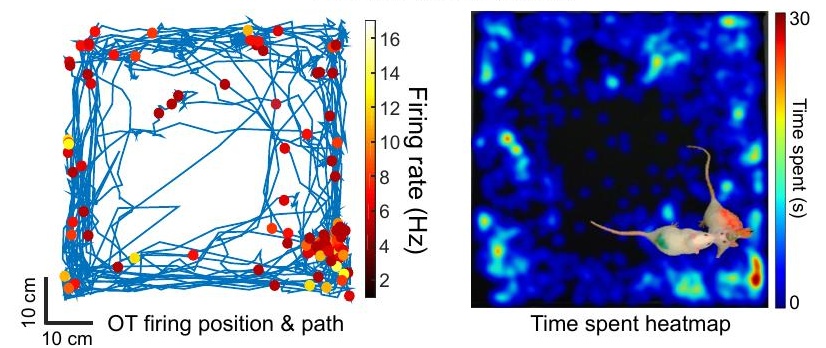

d $\square$ Open field $\square$ No interaction $\square$ Free Social Interaction
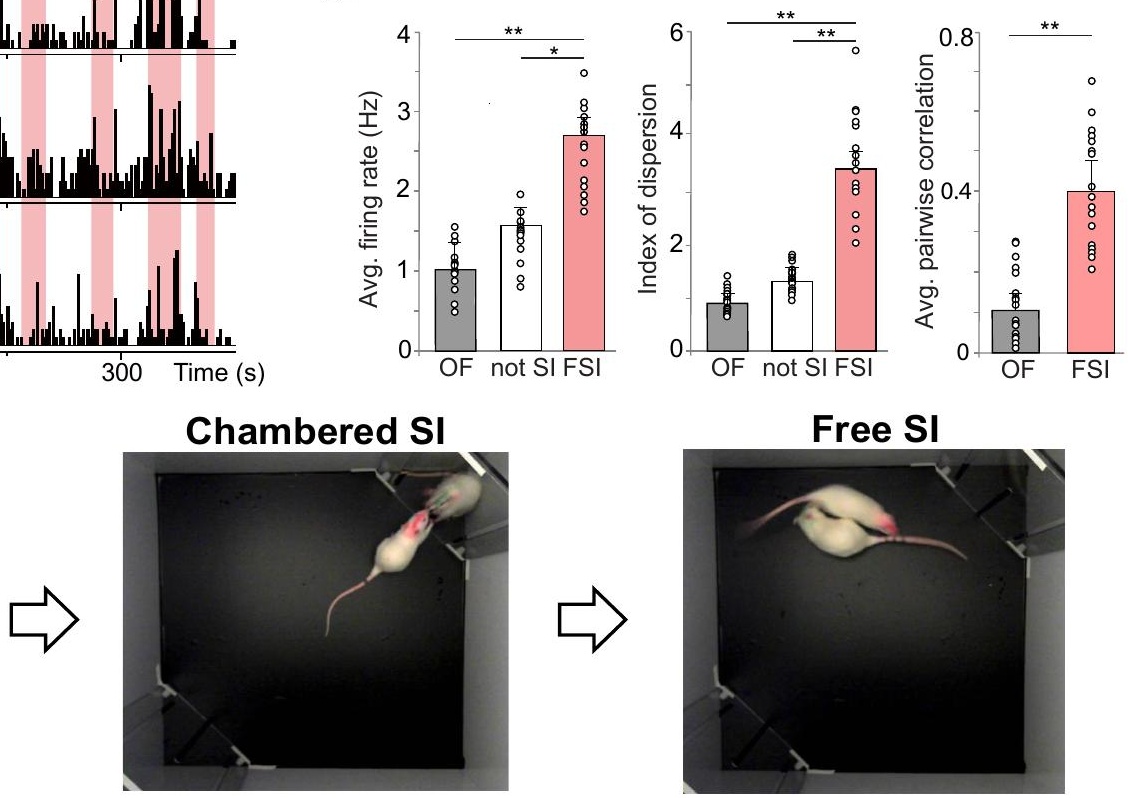

Average firing rate $1.4 \mathrm{~Hz}$ ||||||||||||||||||||||||||||||||||||||||||||||||||||||||||||
Free SI

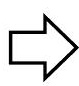

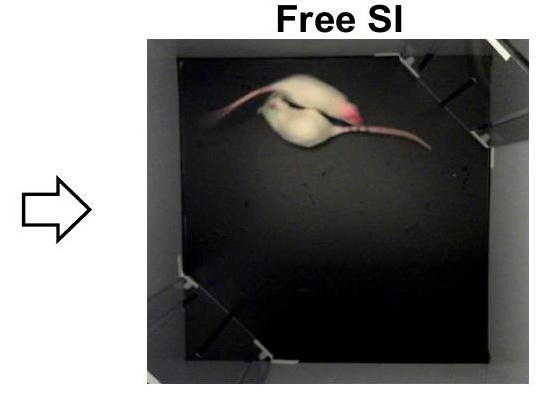

Average firing rate $2.9 \mathrm{~Hz}$ |||||||||||||||||||||||||||||||||||||||||||||||||||||||||||||

f
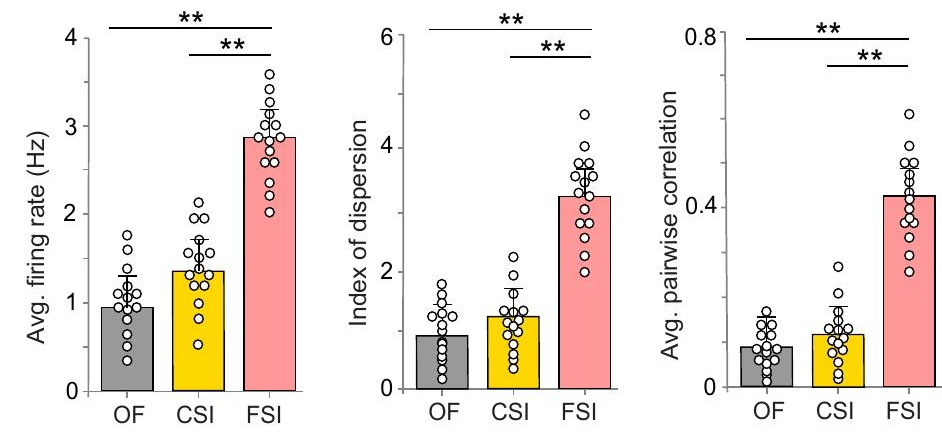

g

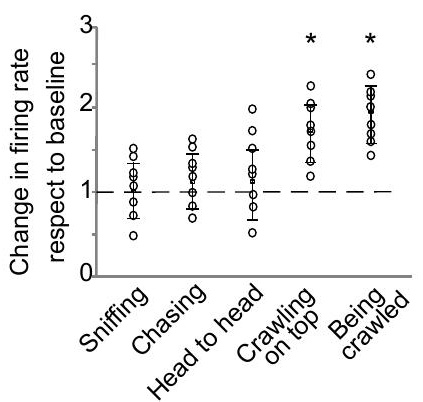

h
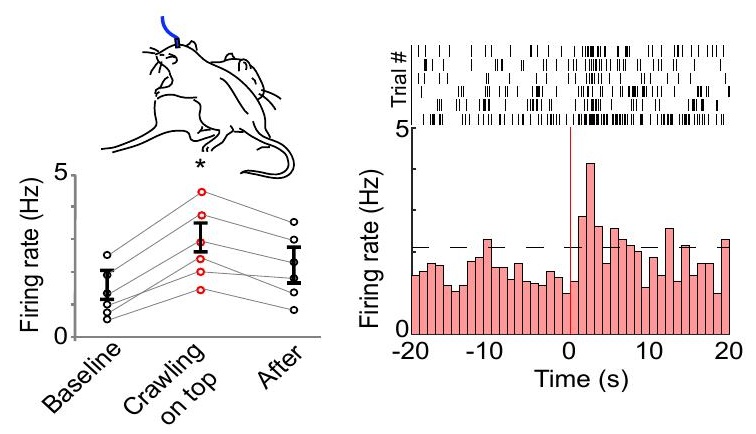
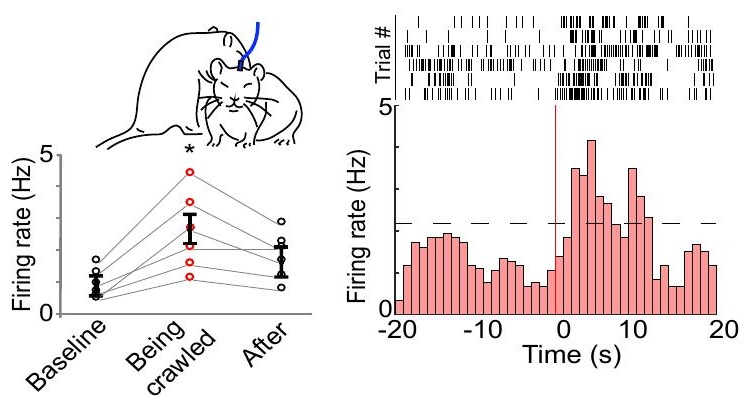
Figure 2

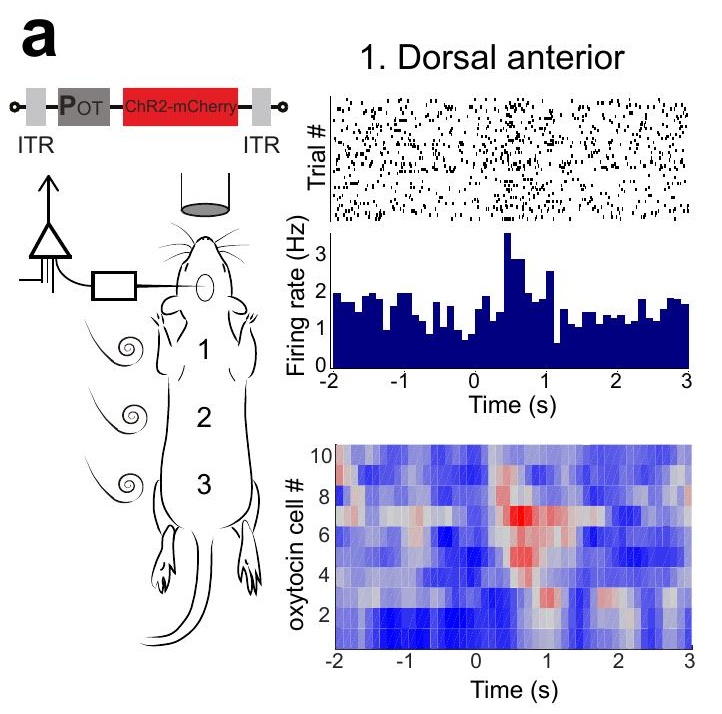

2. Dorsal central
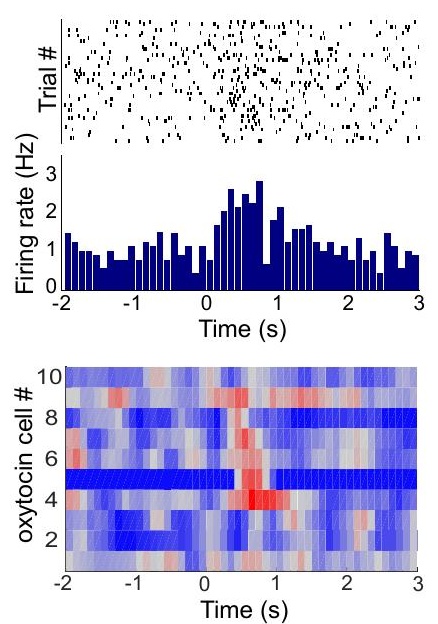

d

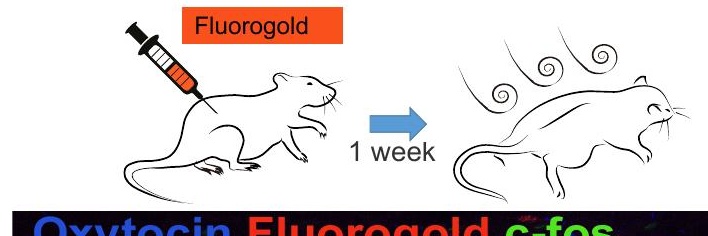

Oxytocin Fluorogold c-fos

e

g

C

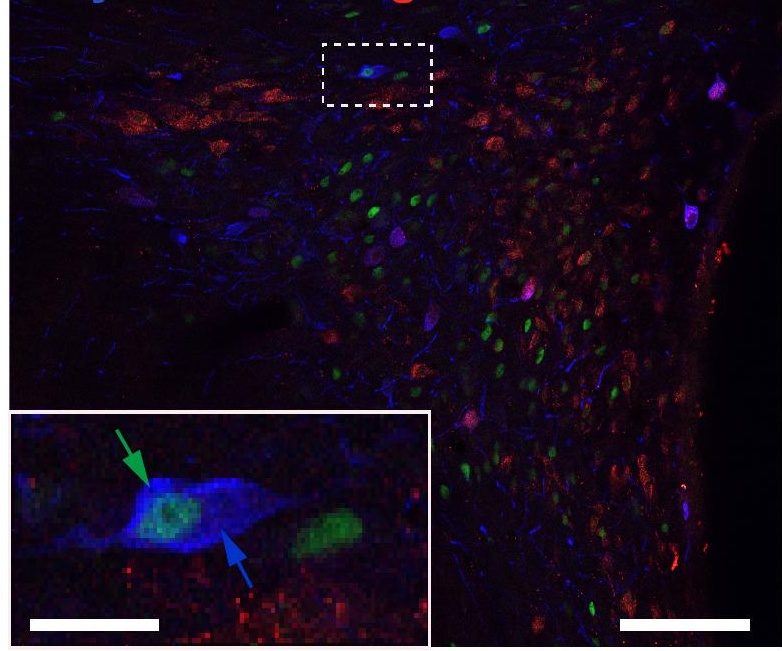

e

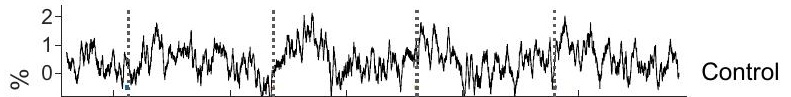

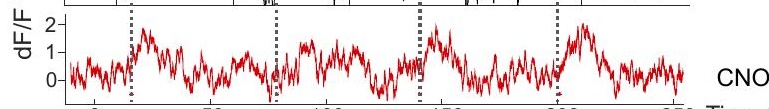

I
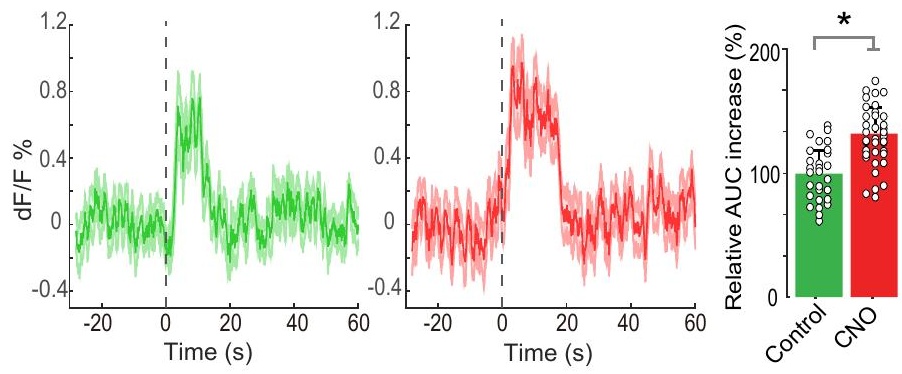

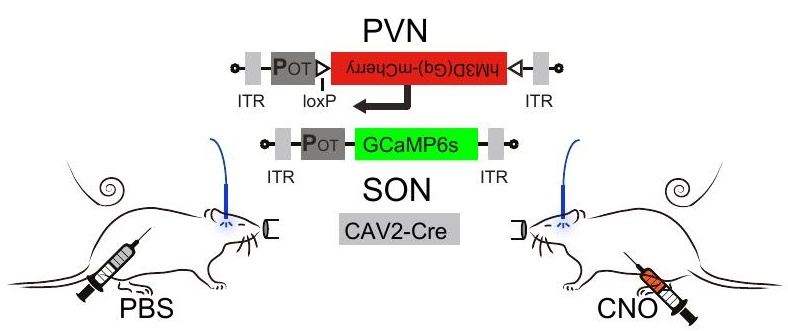

3. Dorsal posterior

b

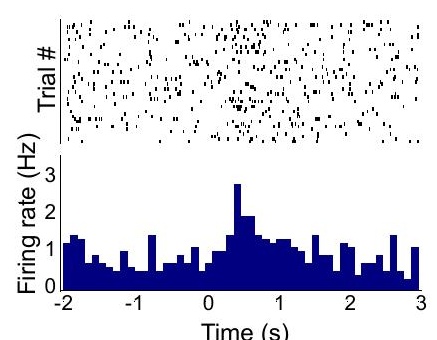

Peak amplitude
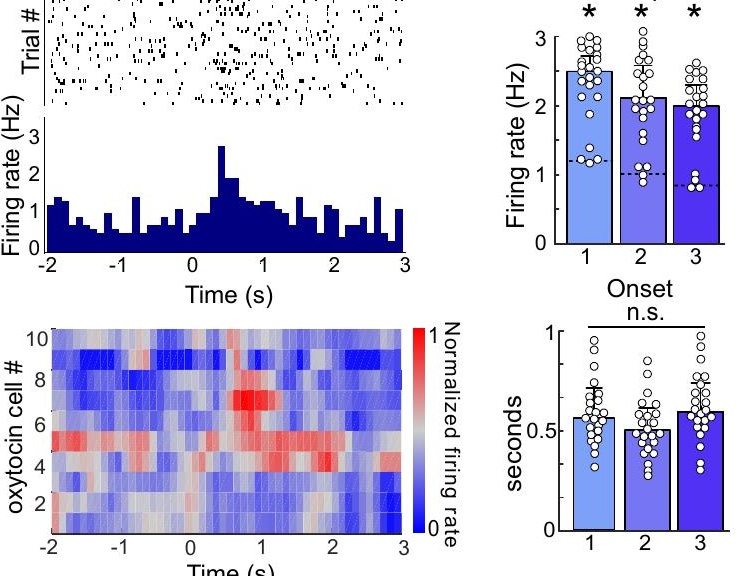
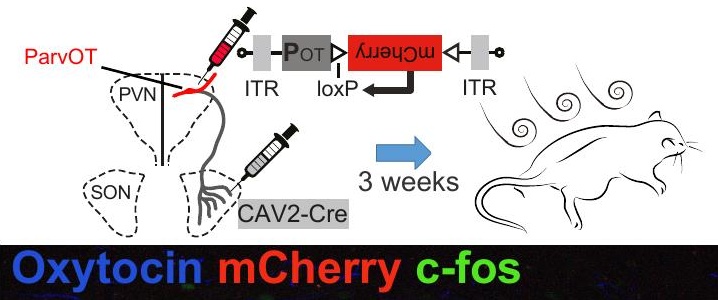

h

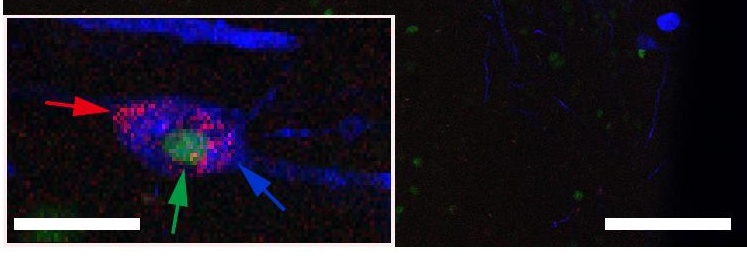

f

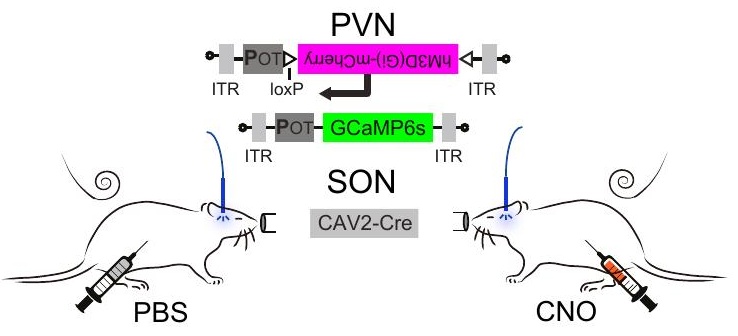

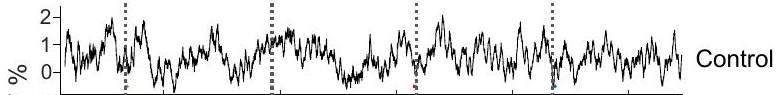

崖 $\begin{gathered}2-1 \\ 0\end{gathered}$

j

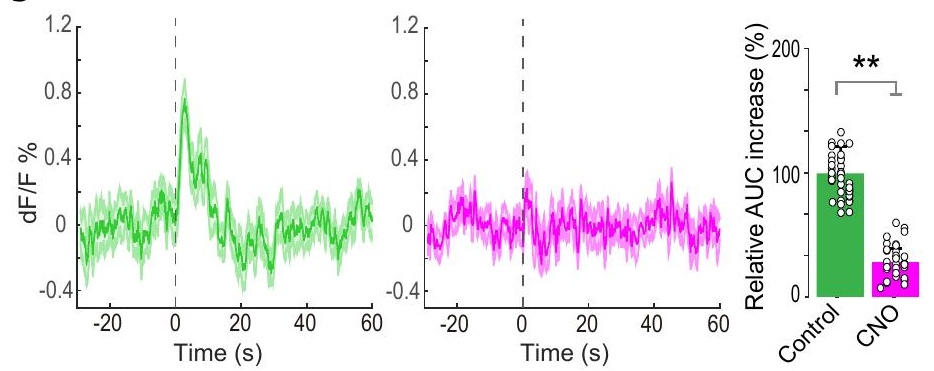


a

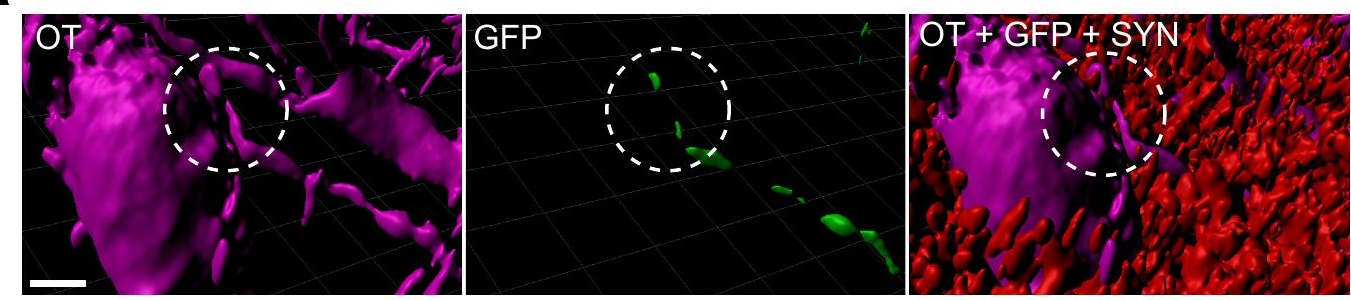

b
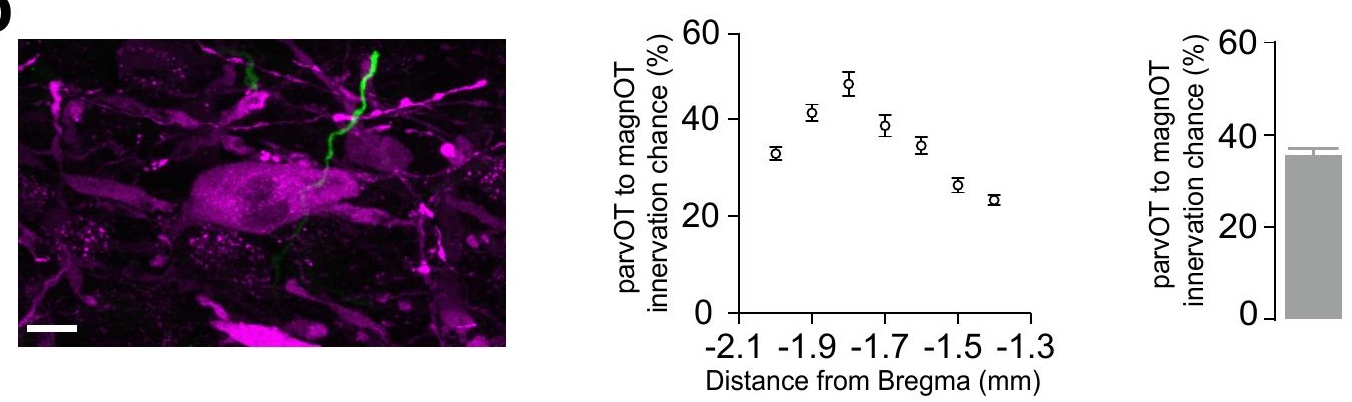

C
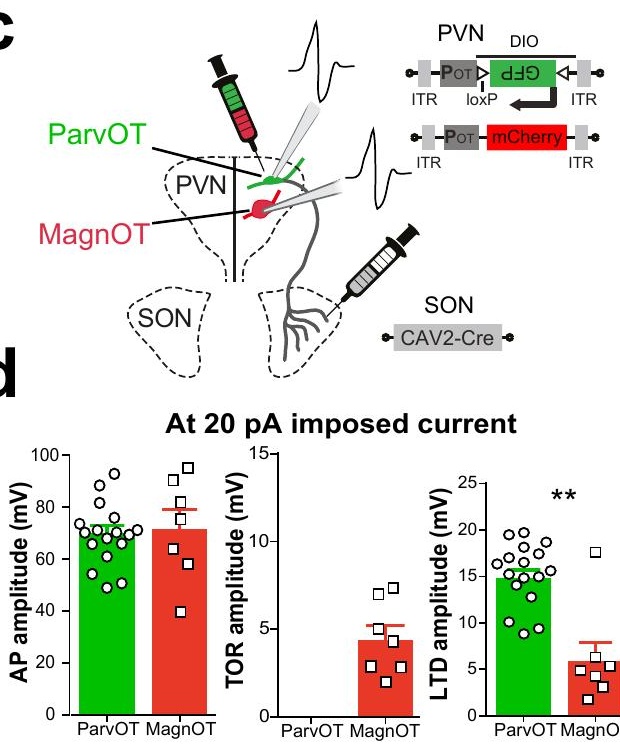

20 pA imposed current

g
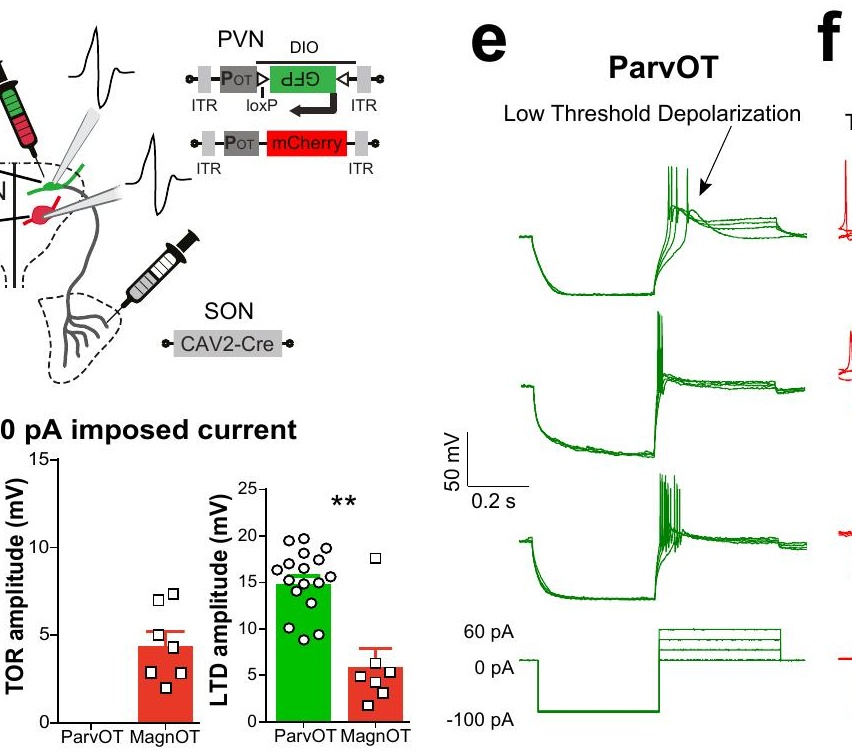

MagnOT
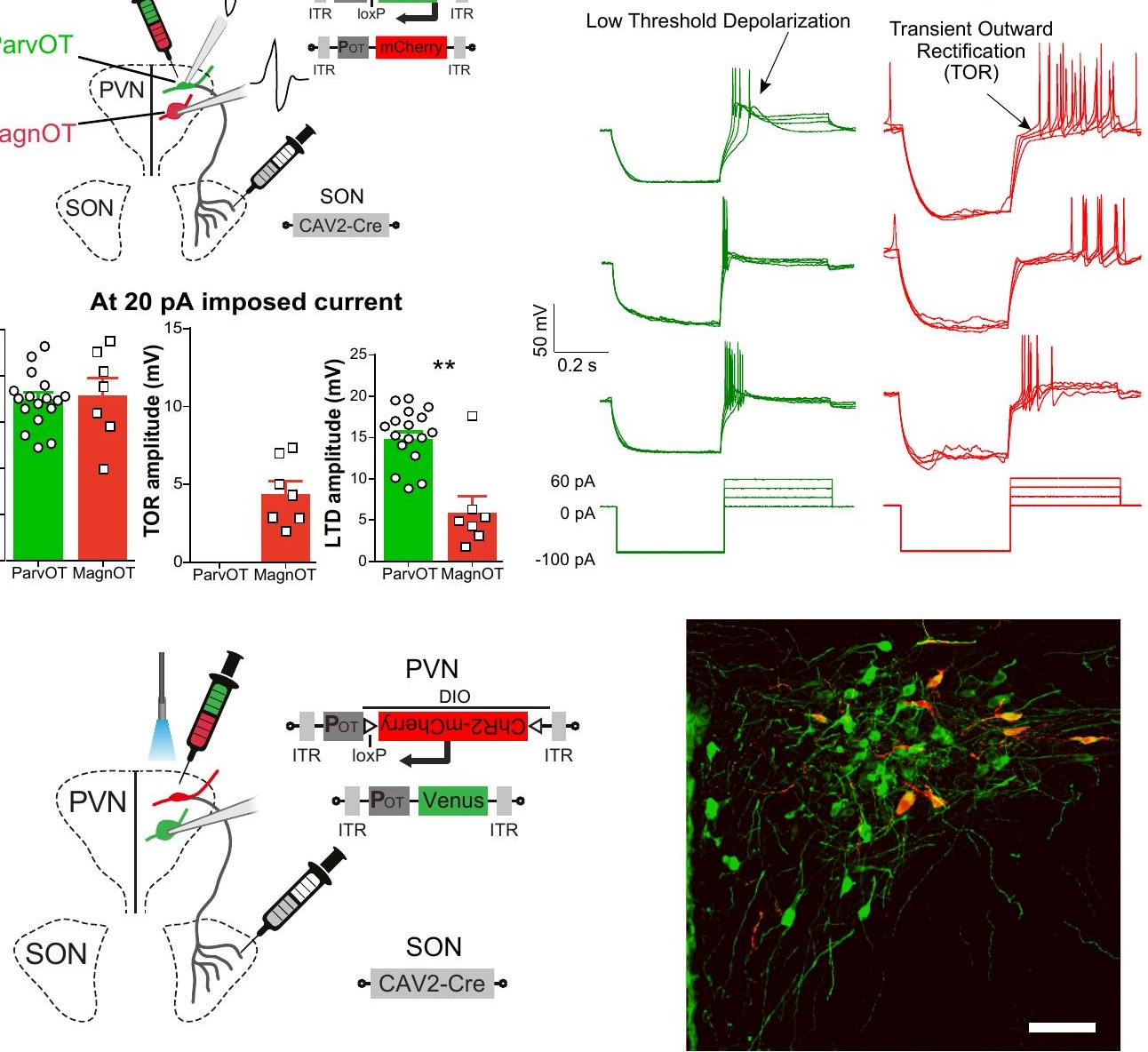

h
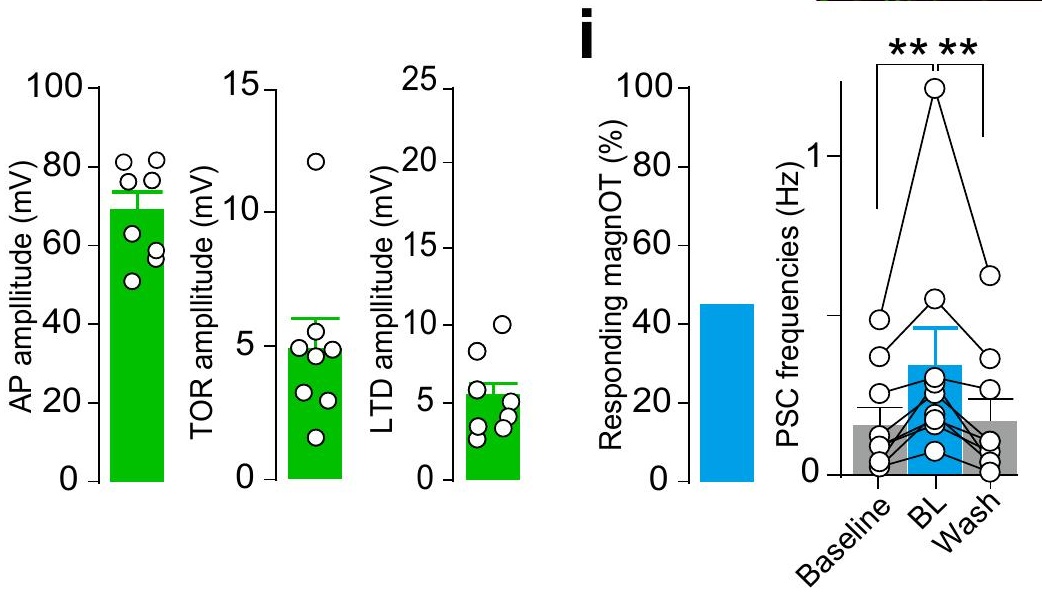

Baseline BL
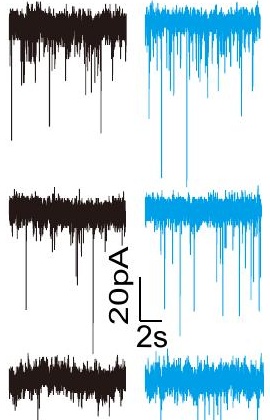
Figure 4

a

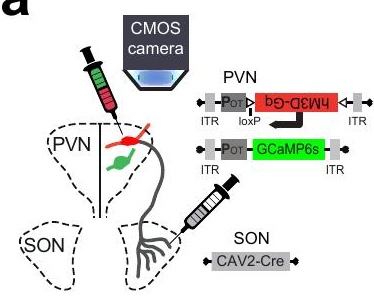

b

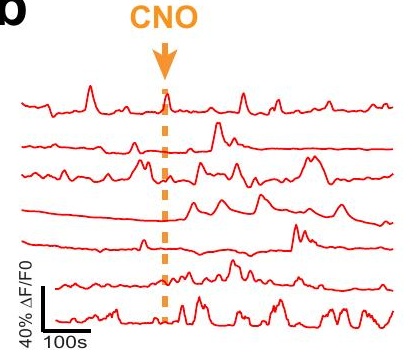

C

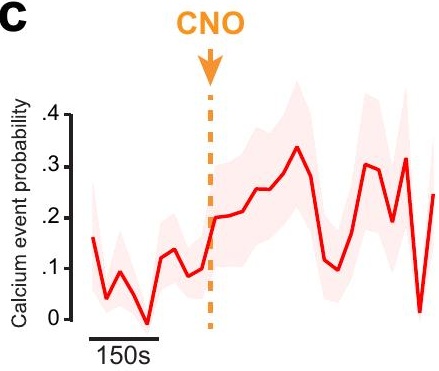

e

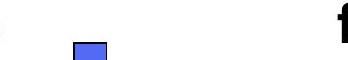
Before CNO

i

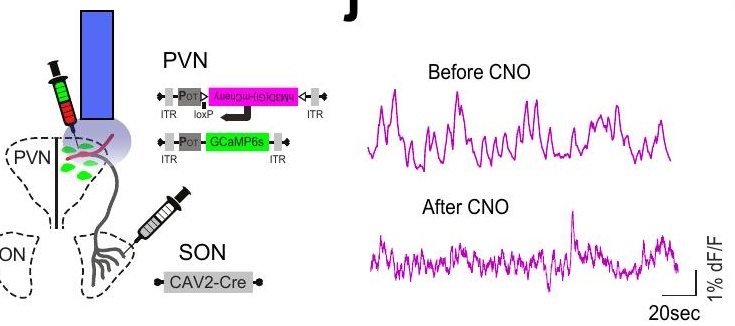

m

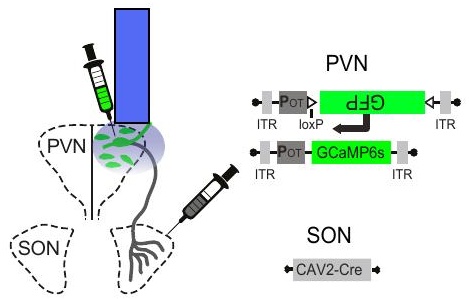

p

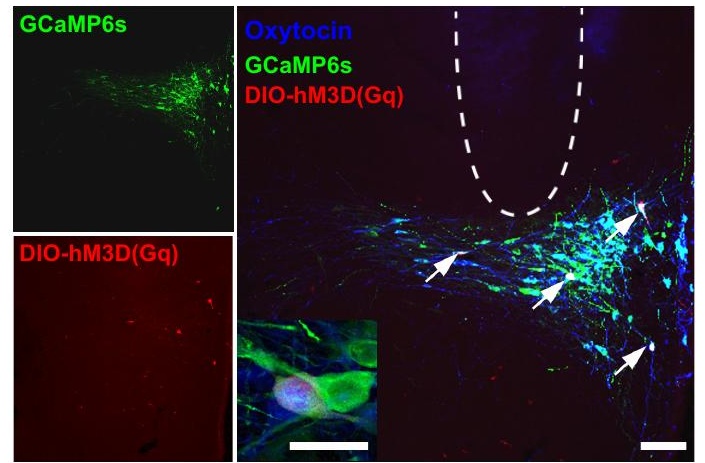

\section{g $\quad$ CNO}
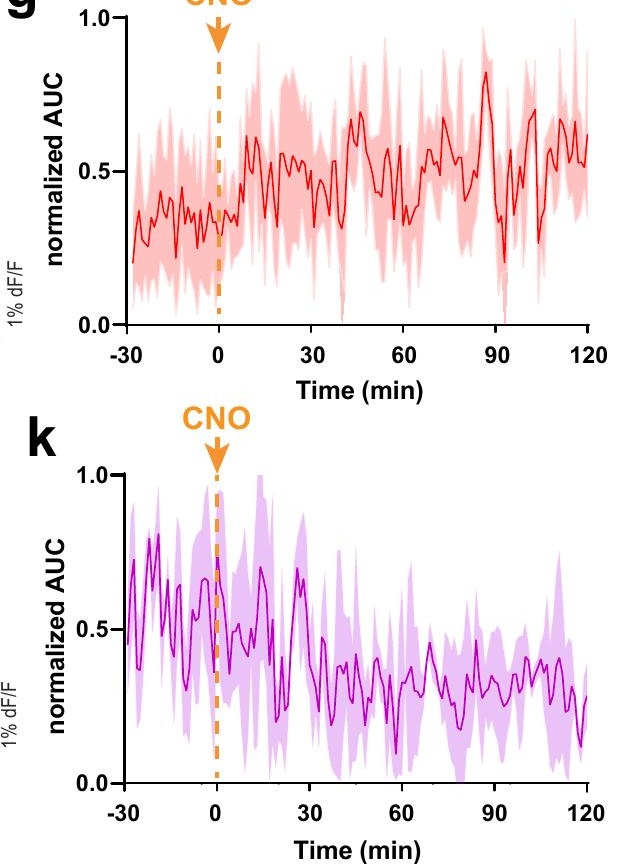

n

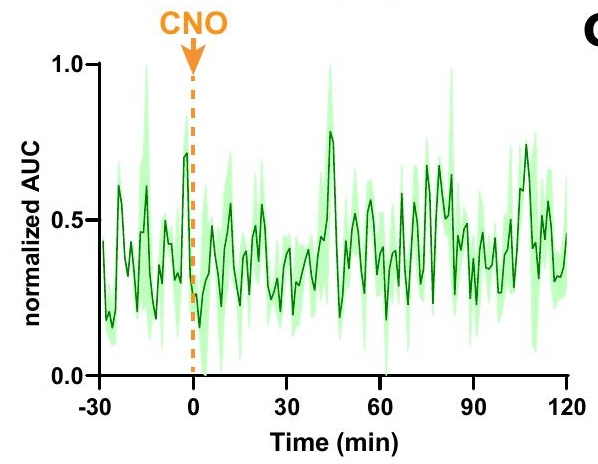

q

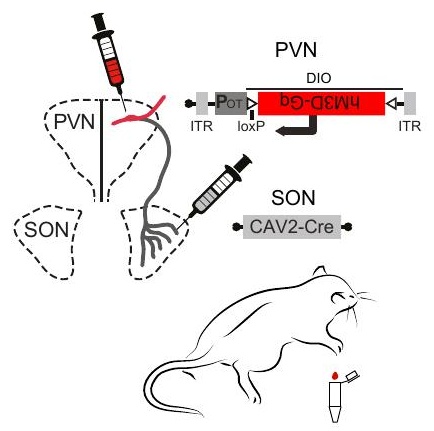

d

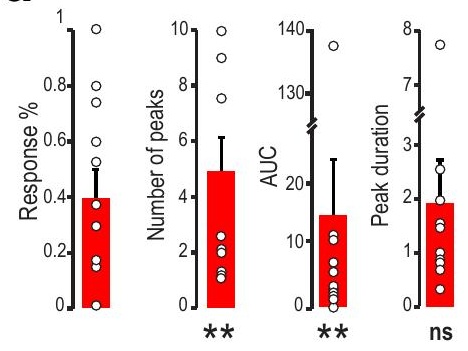

h
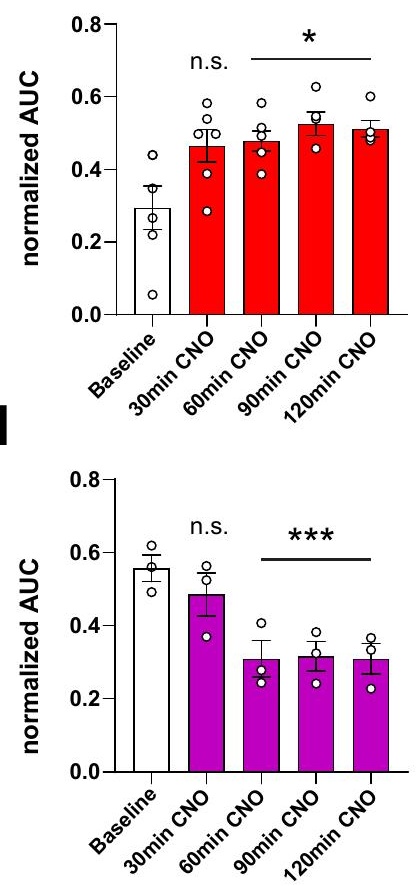

0

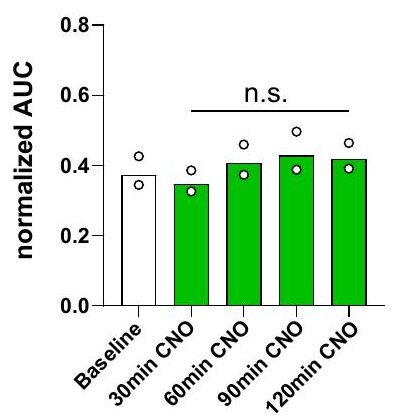

$r$

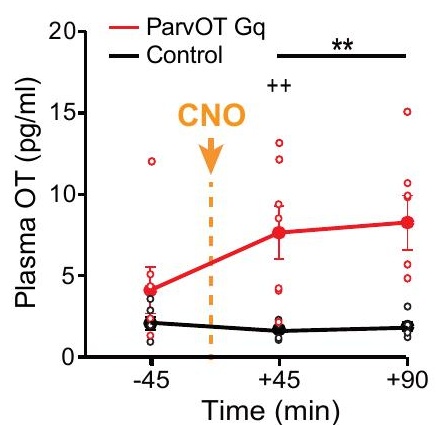


a $\quad$ Figure 5
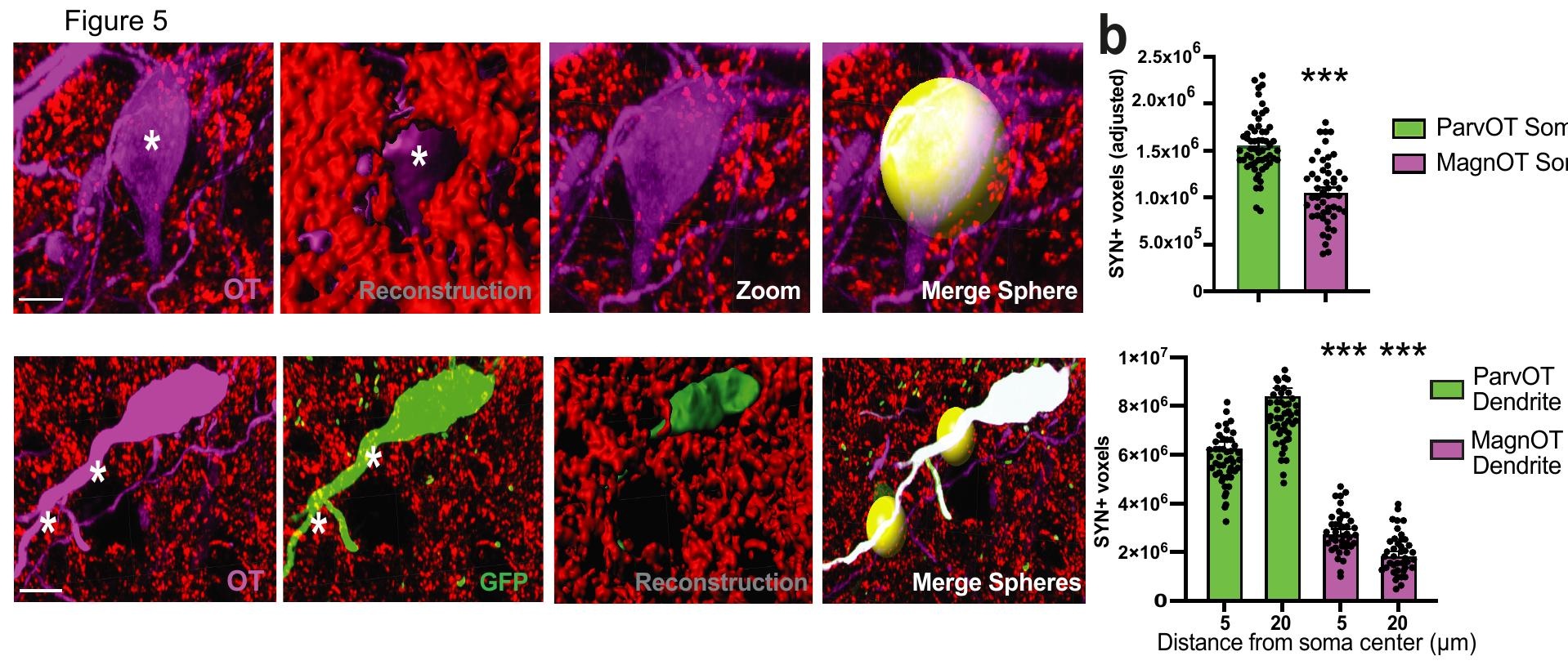

C Inputs to parvOT neurons PVN

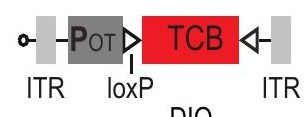
a- Perla $\frac{D I O}{50} 4-0$ ITR $\operatorname{loxP} \sqcup$ ITR 14 days

o- Rb-GFP -o SON - CAV2-Cre -

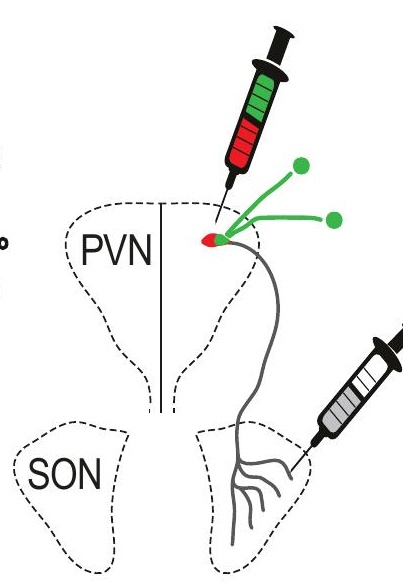

e

Inputs to magnOT PVN$$
\text { ITR }
$$

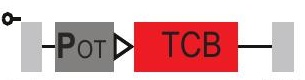

ITR$$
\text { ITR }
$$

- $-P_{\text {ef1a }} \frac{D I O}{50 \triangleleft-0}$ ITR loxP o- 14 days

Posterior Pituitary o- AAVretro-Cre -0

g
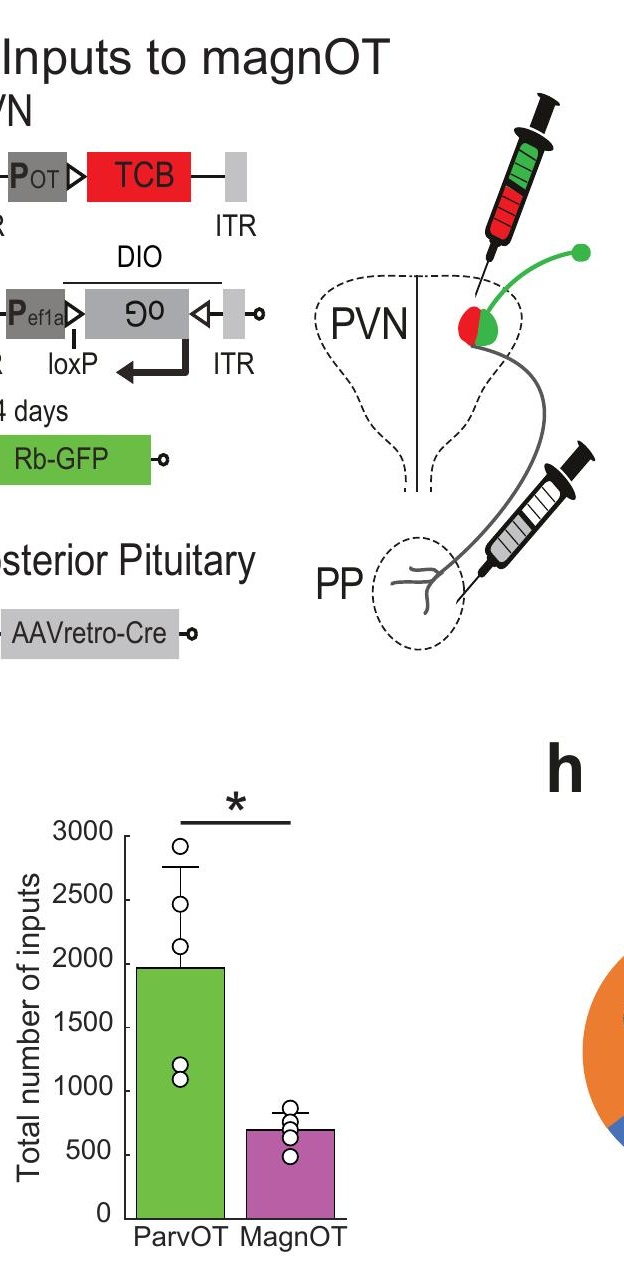

h d

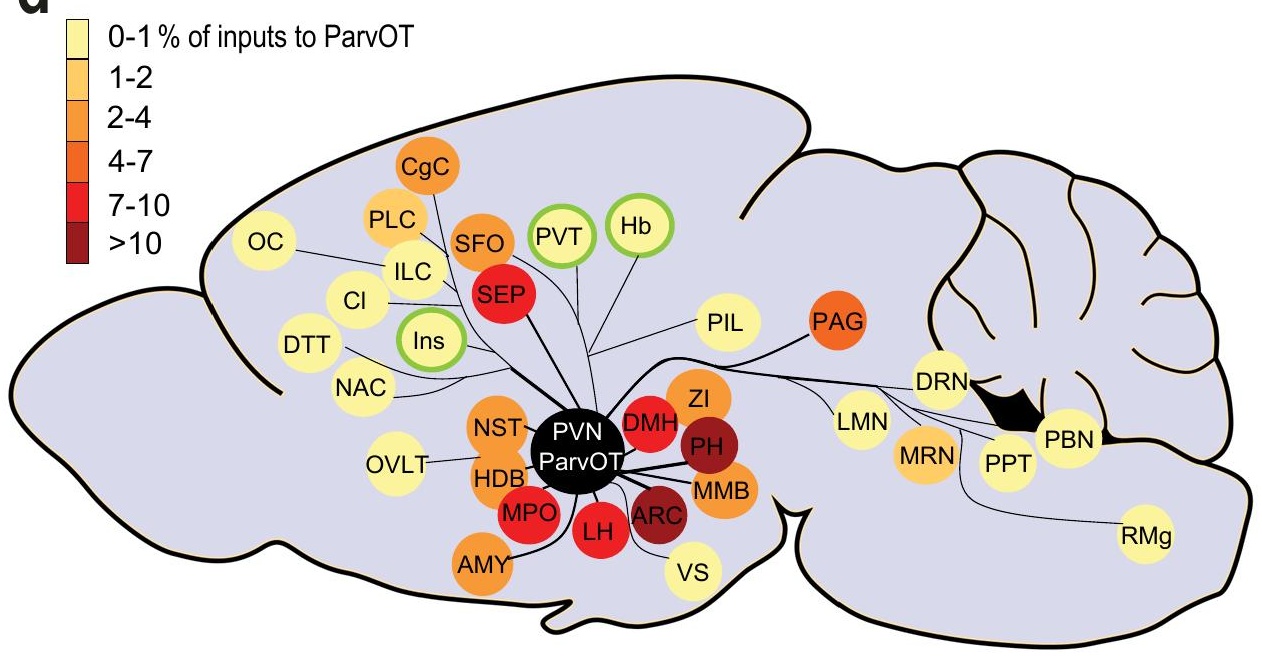

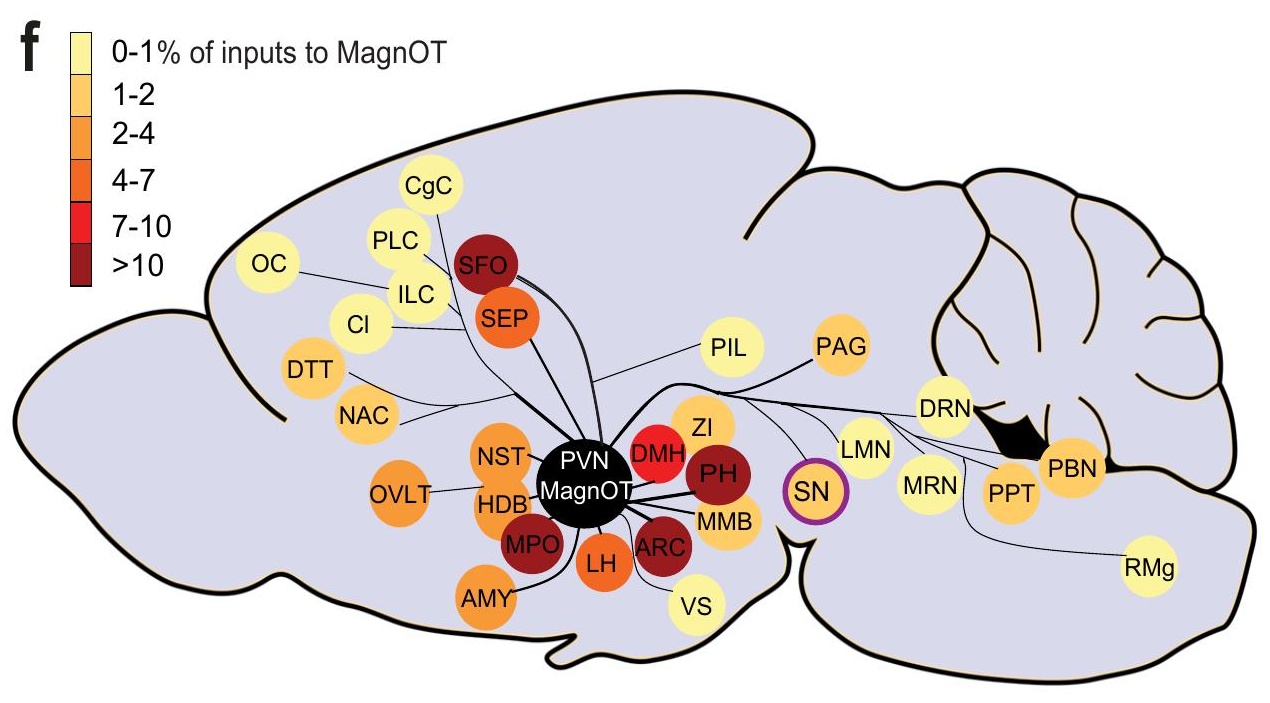
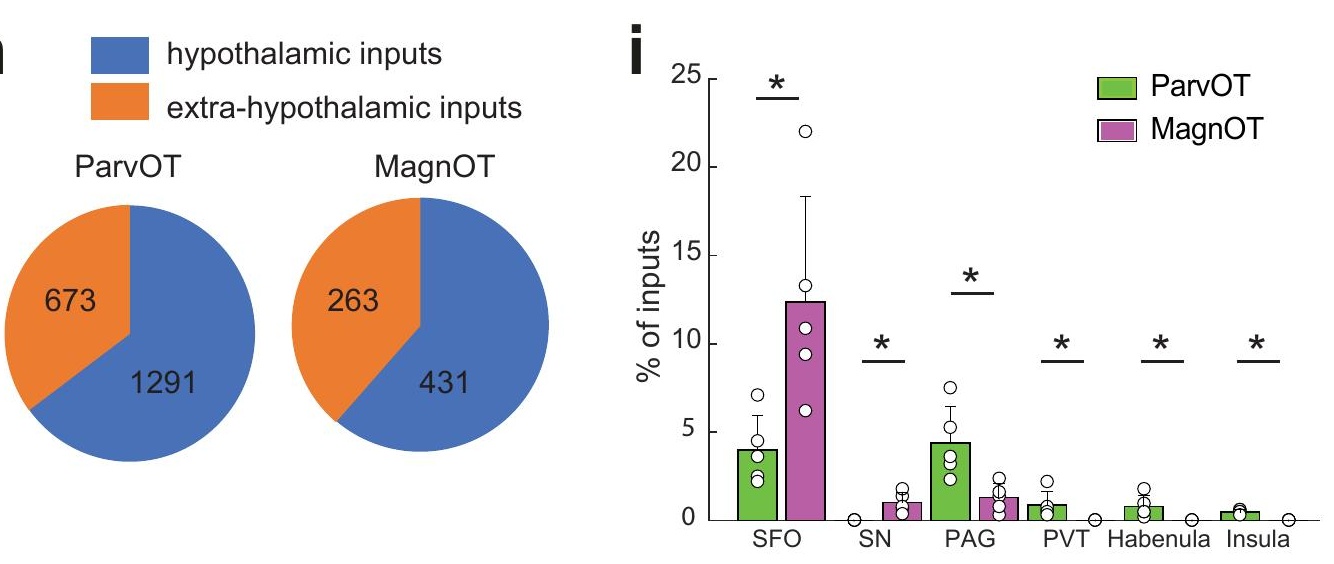
Figure 6

a

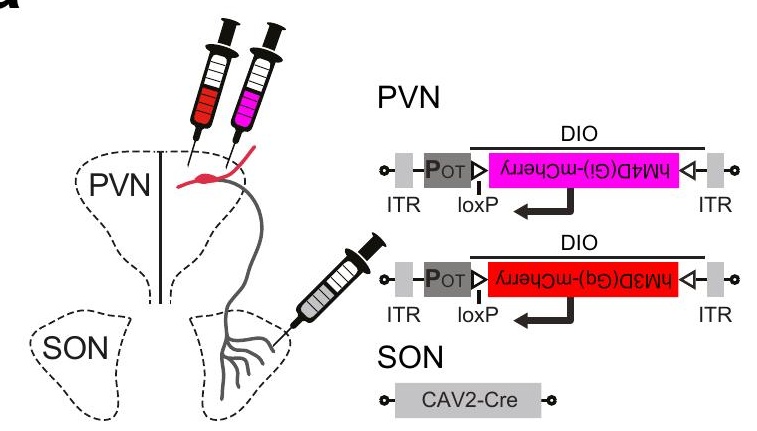

b

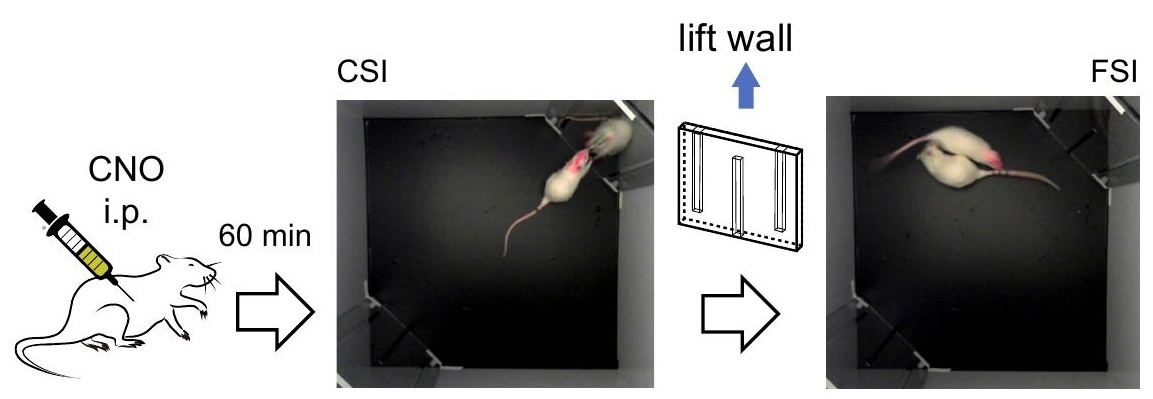

C

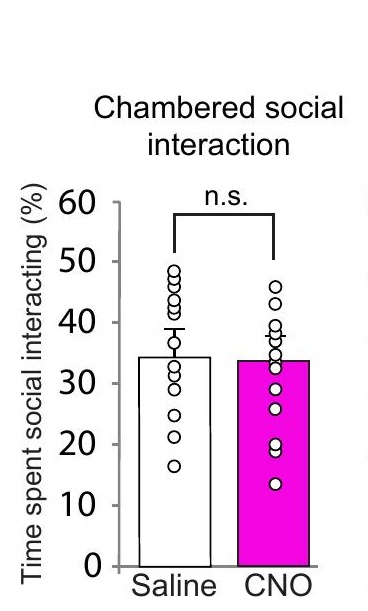

f

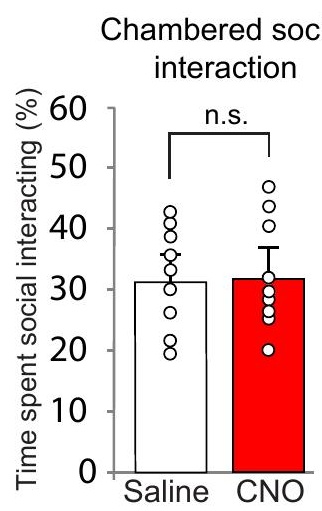

Free social

interaction

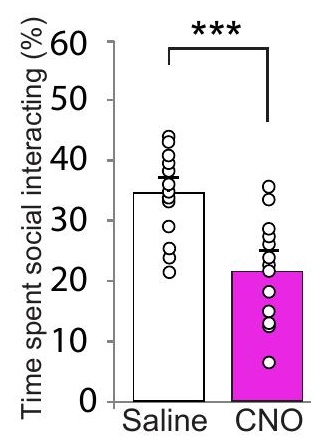

\section{g}

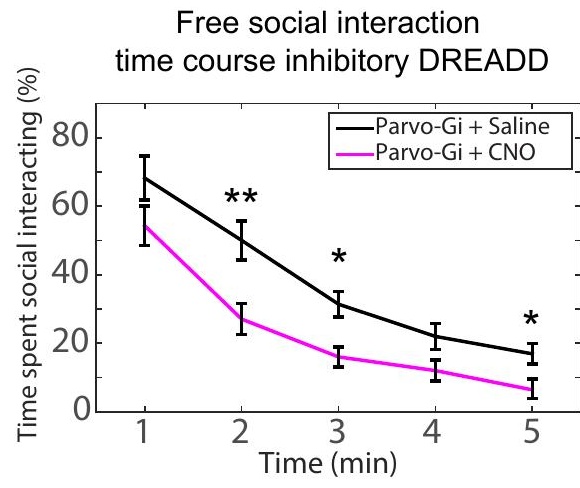

d

e

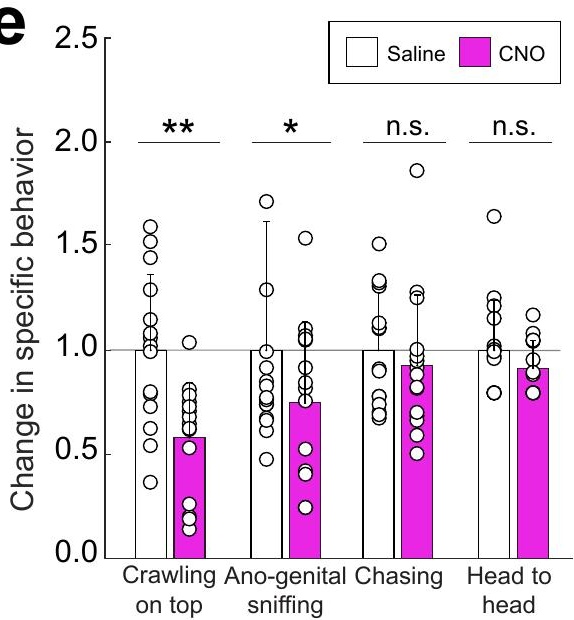

h

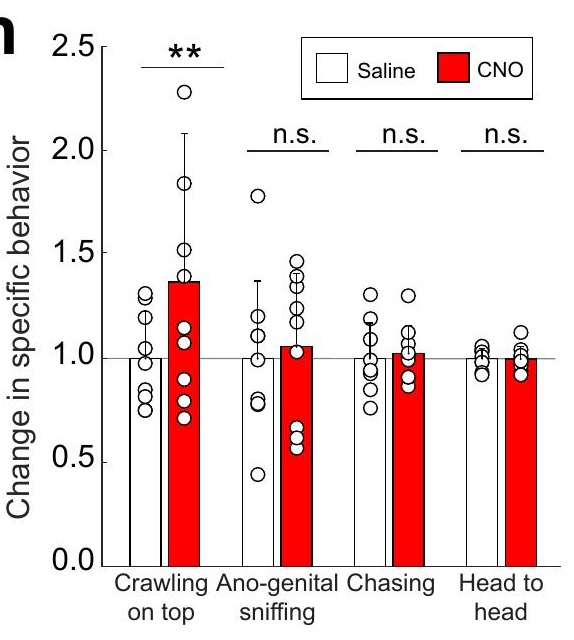

i
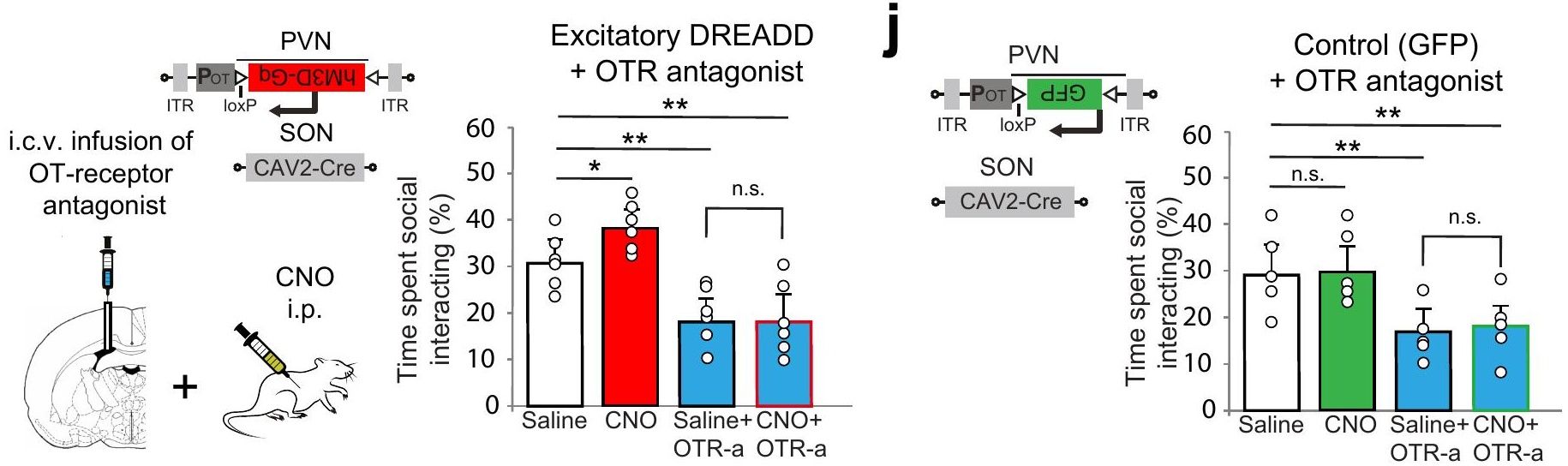
Ano-genital sniffing

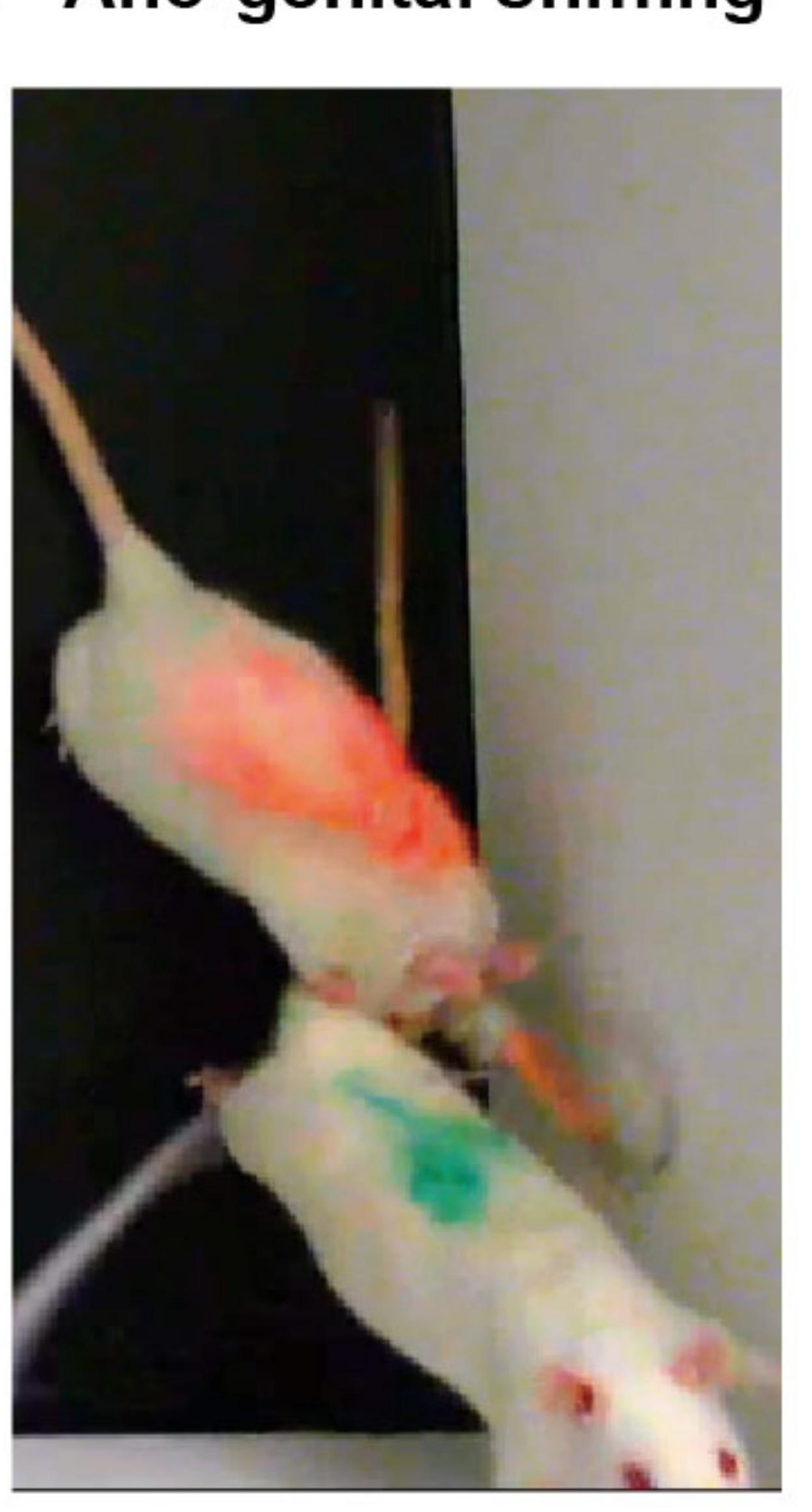

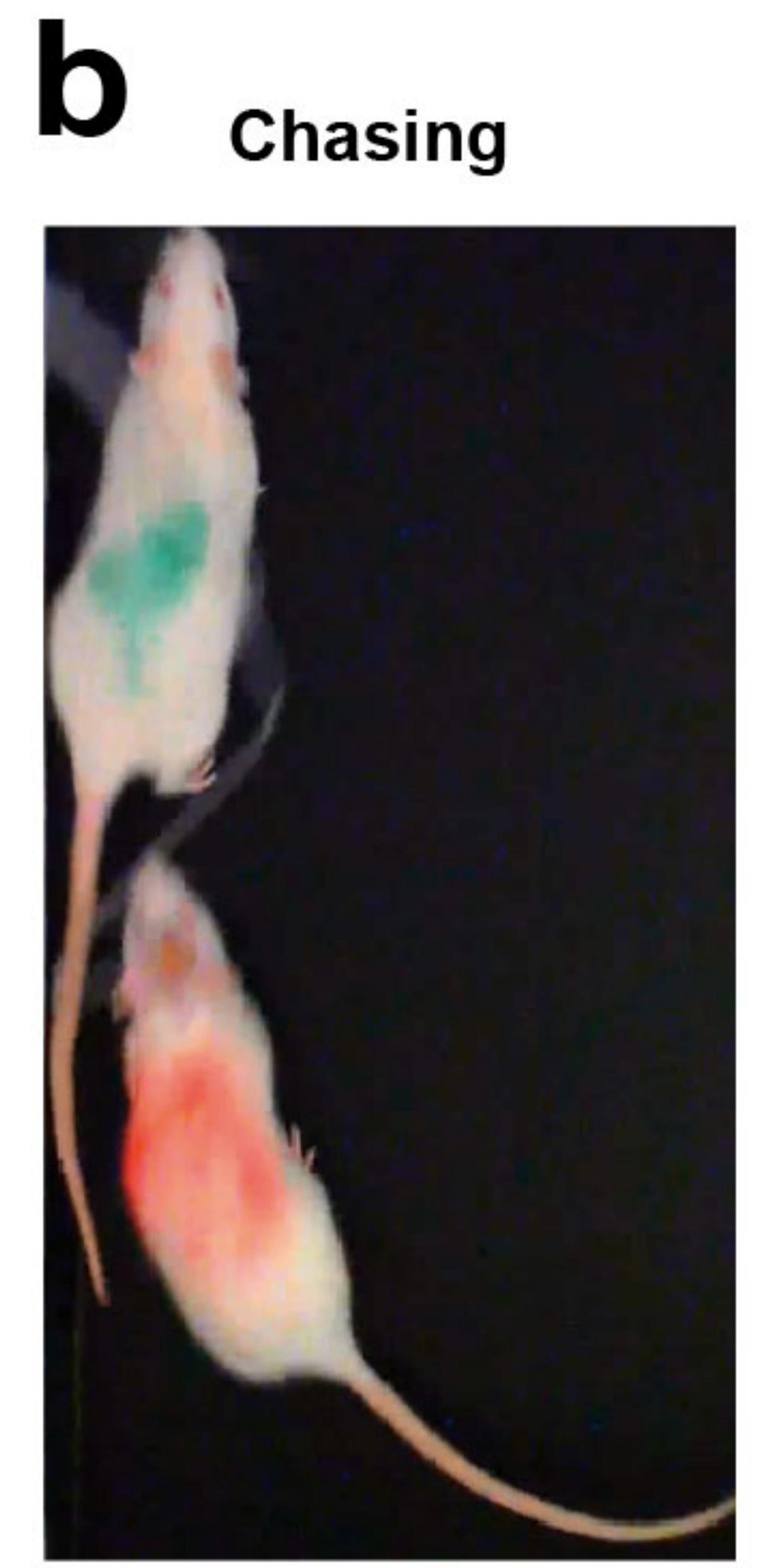

\section{Head-head}
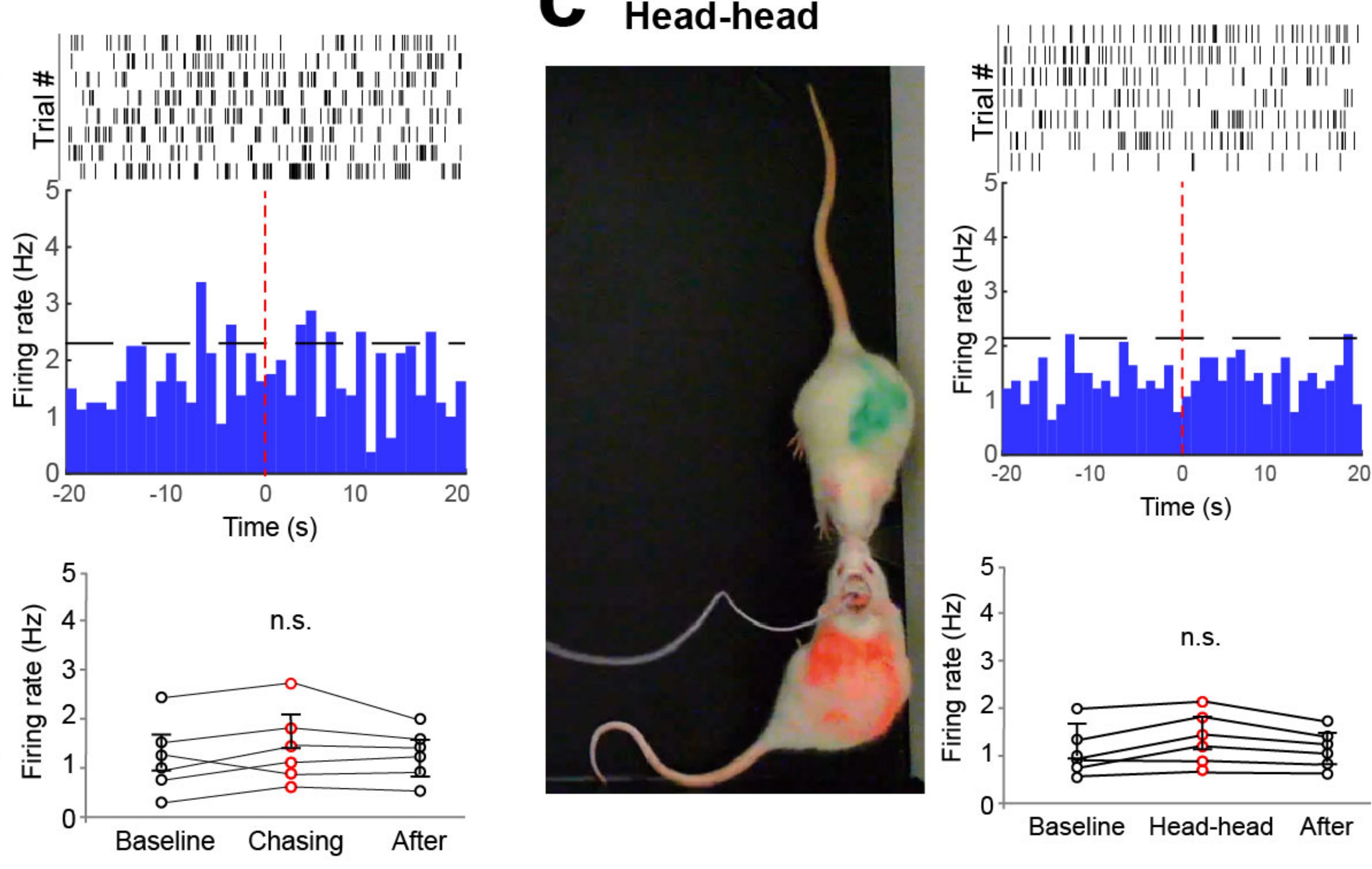

e
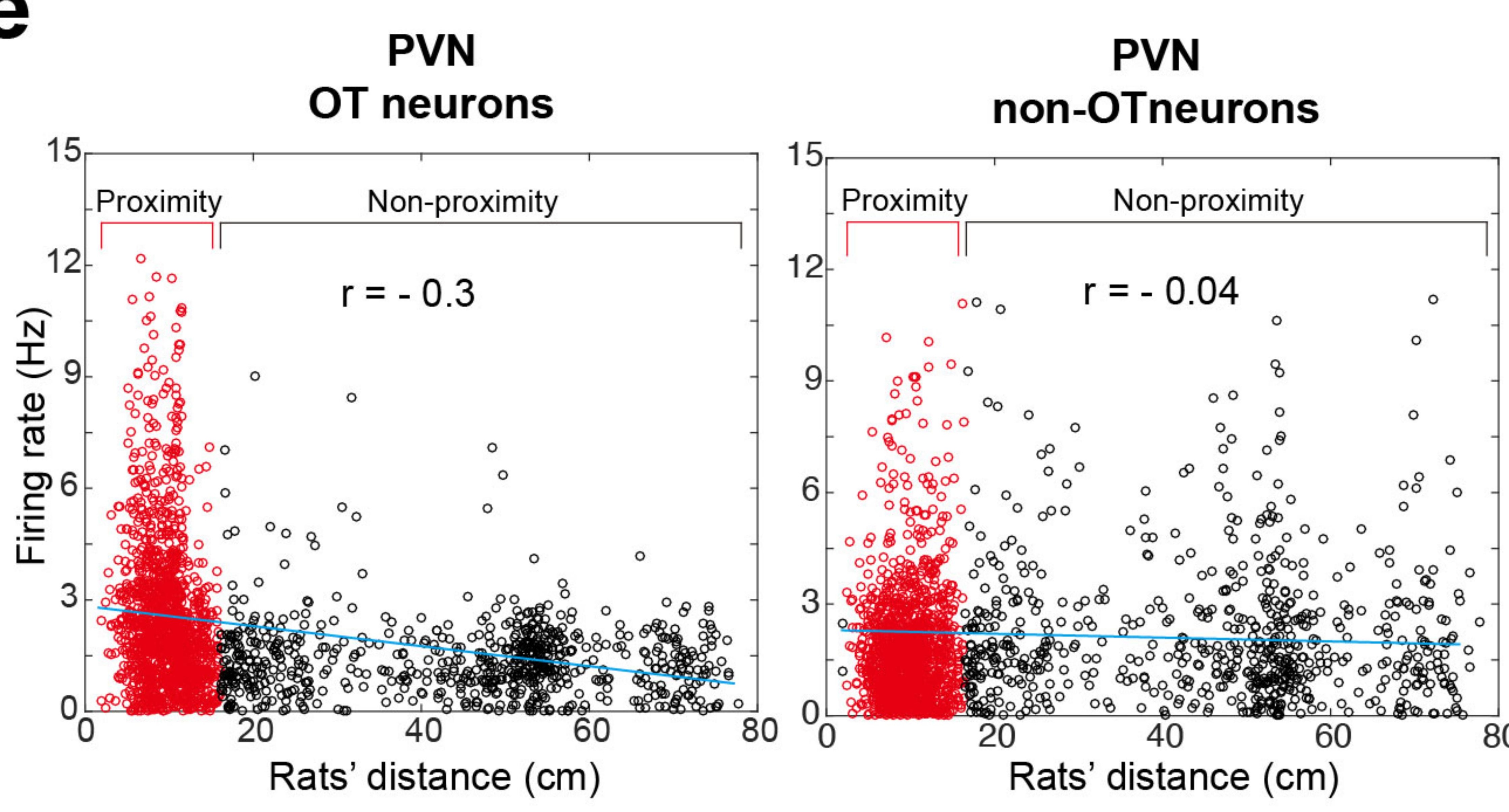

g 15

5
5
0
0
0

\section{PVN non-OT neurons}

$\square$ Open field $\square$ Not interacting $\square$ Socially interacting

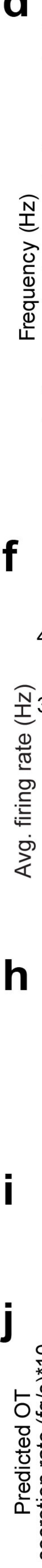

n.s.

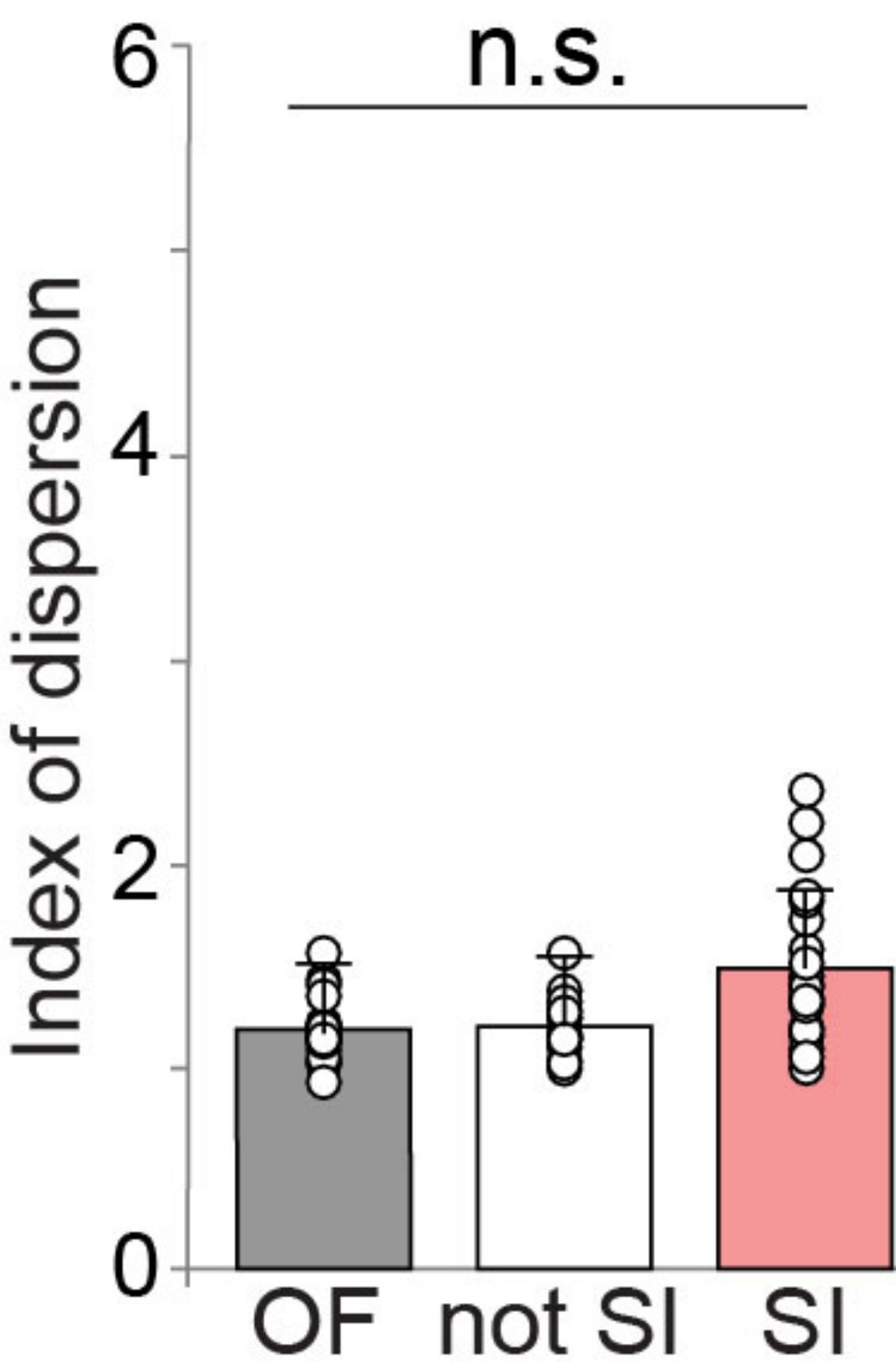

Measured spike pattern

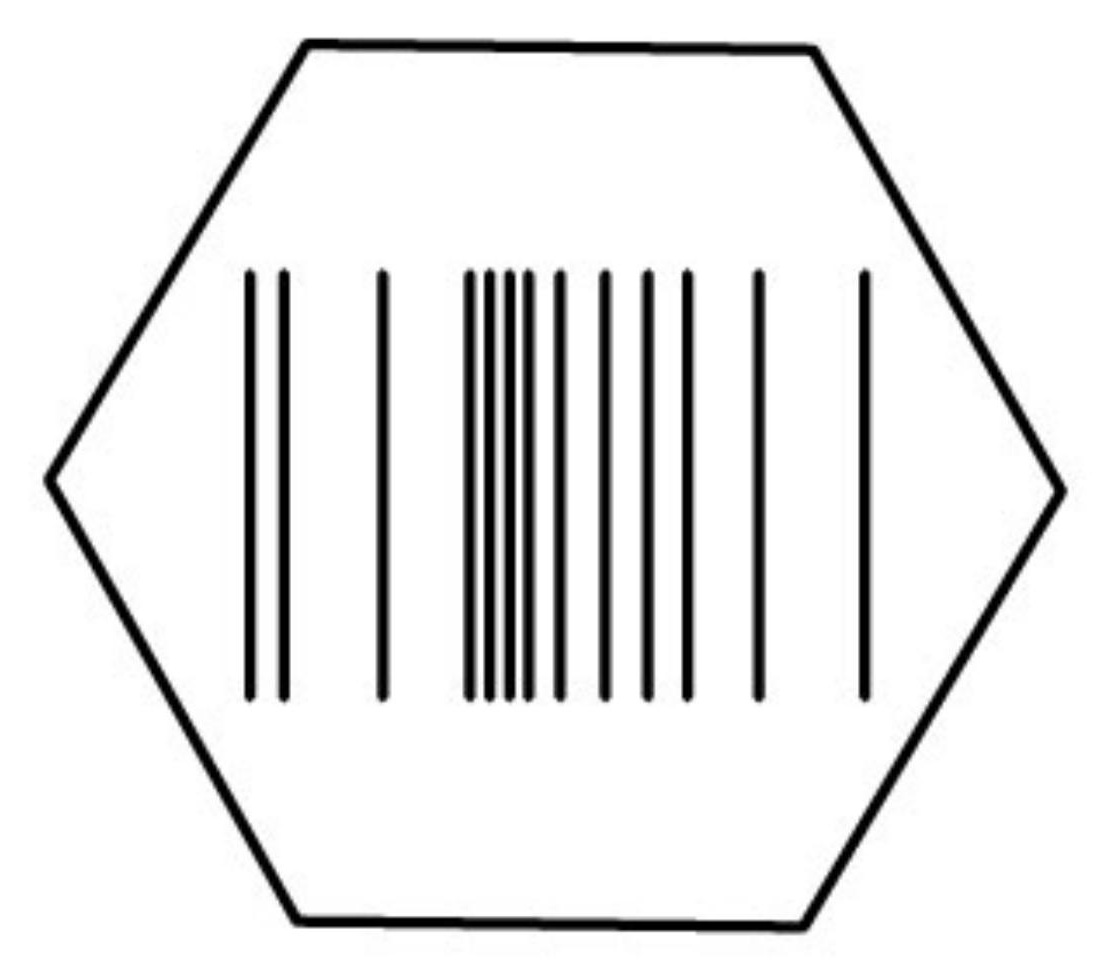

Open Field

$\stackrel{\otimes}{\overline{0}}$ Average firing rate $1.0 \mathrm{~Hz}$$$
\text { เั }
$$

j

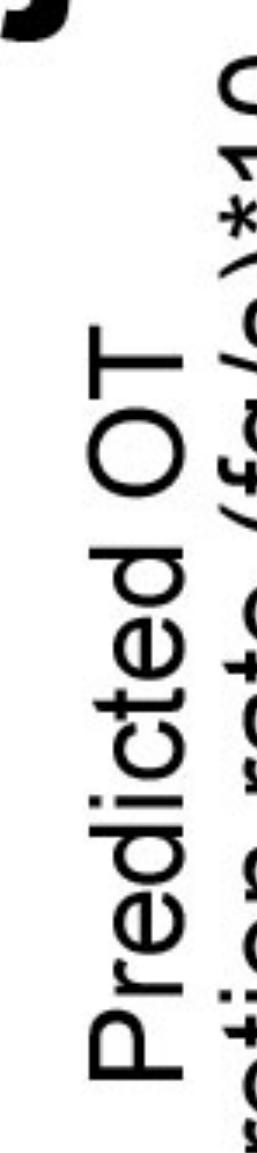

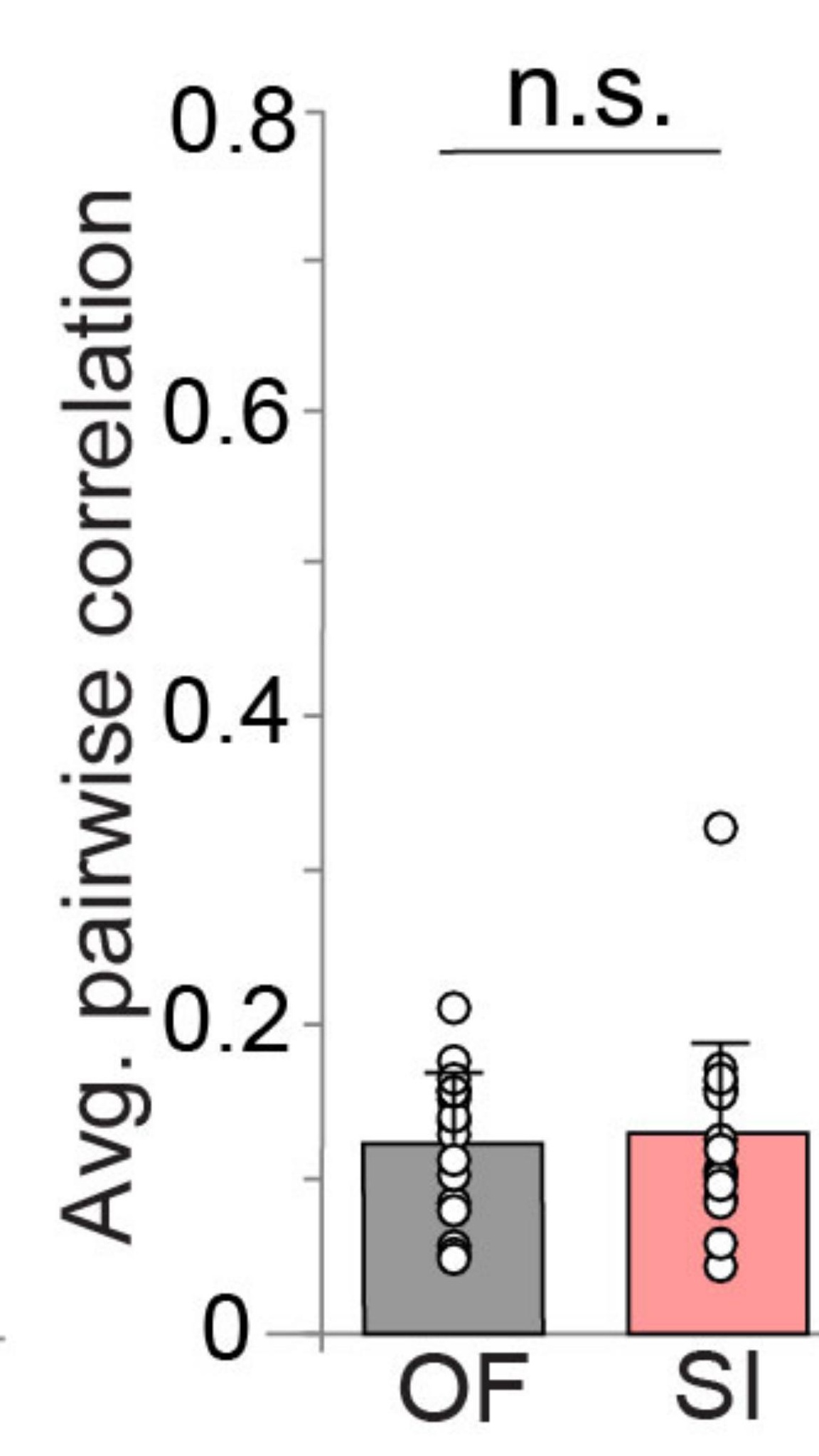

PVN non-OT neurons (Social Interaction)

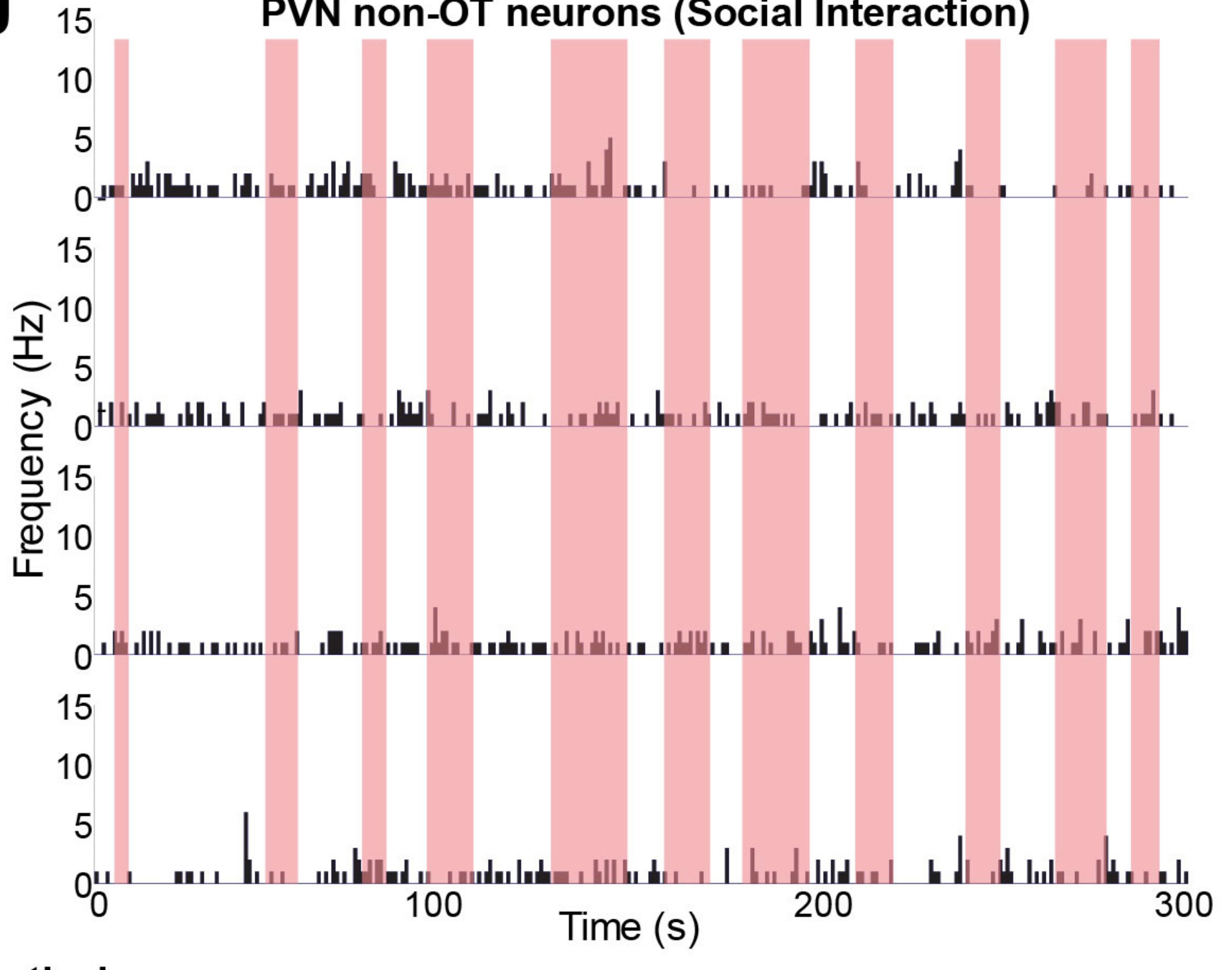

Theoretical

oxytocin secretion model

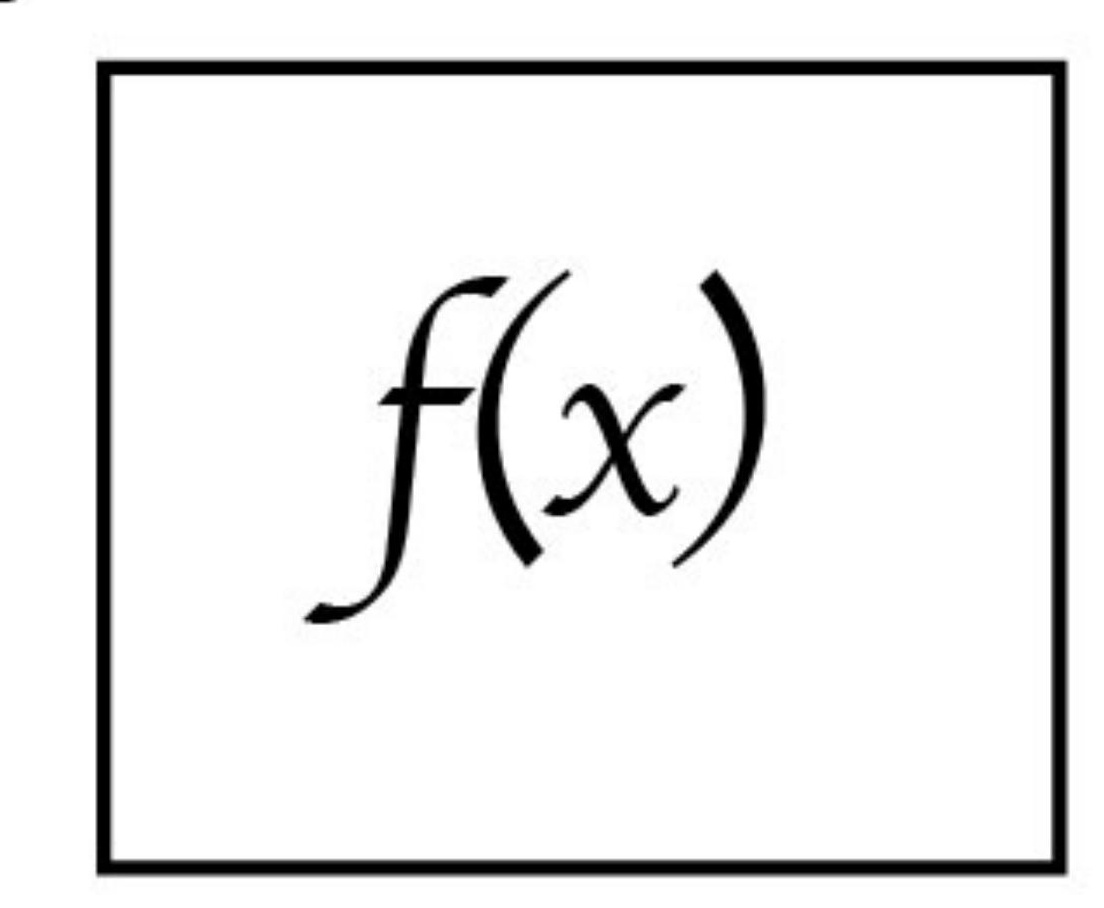

Chambered SI

Average firing rate $1.4 \mathrm{~Hz}$

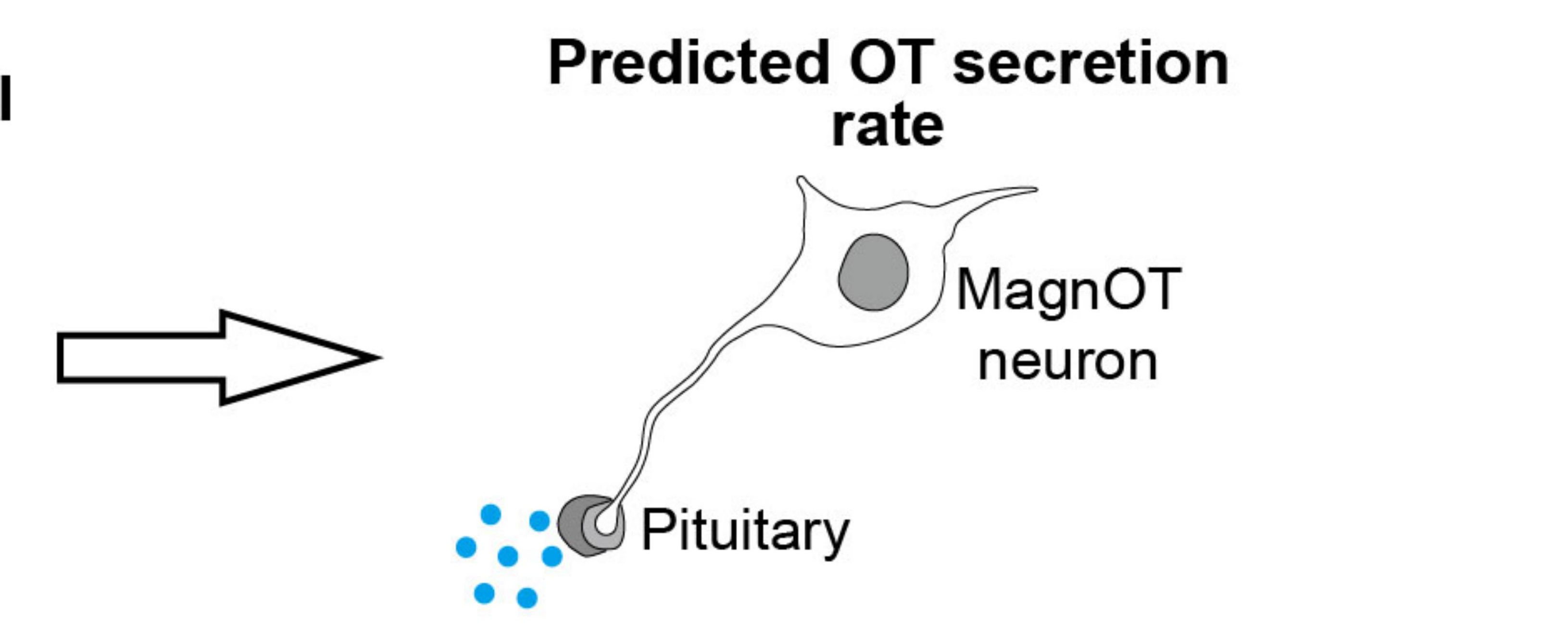

Free SI

Average firing rate $2.9 \mathrm{~Hz}$

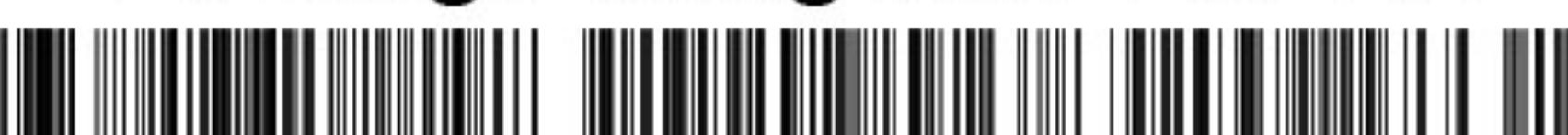

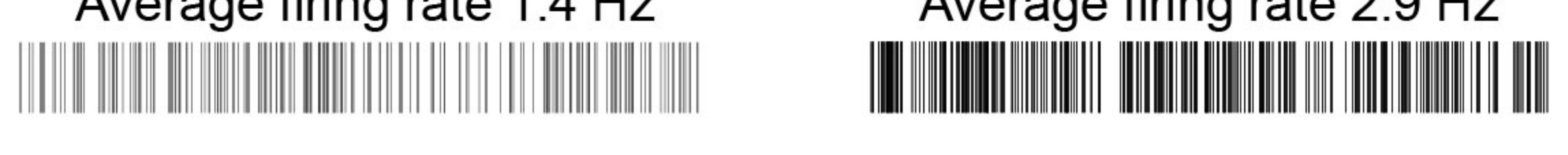

Theoretical oxytocin secretion model
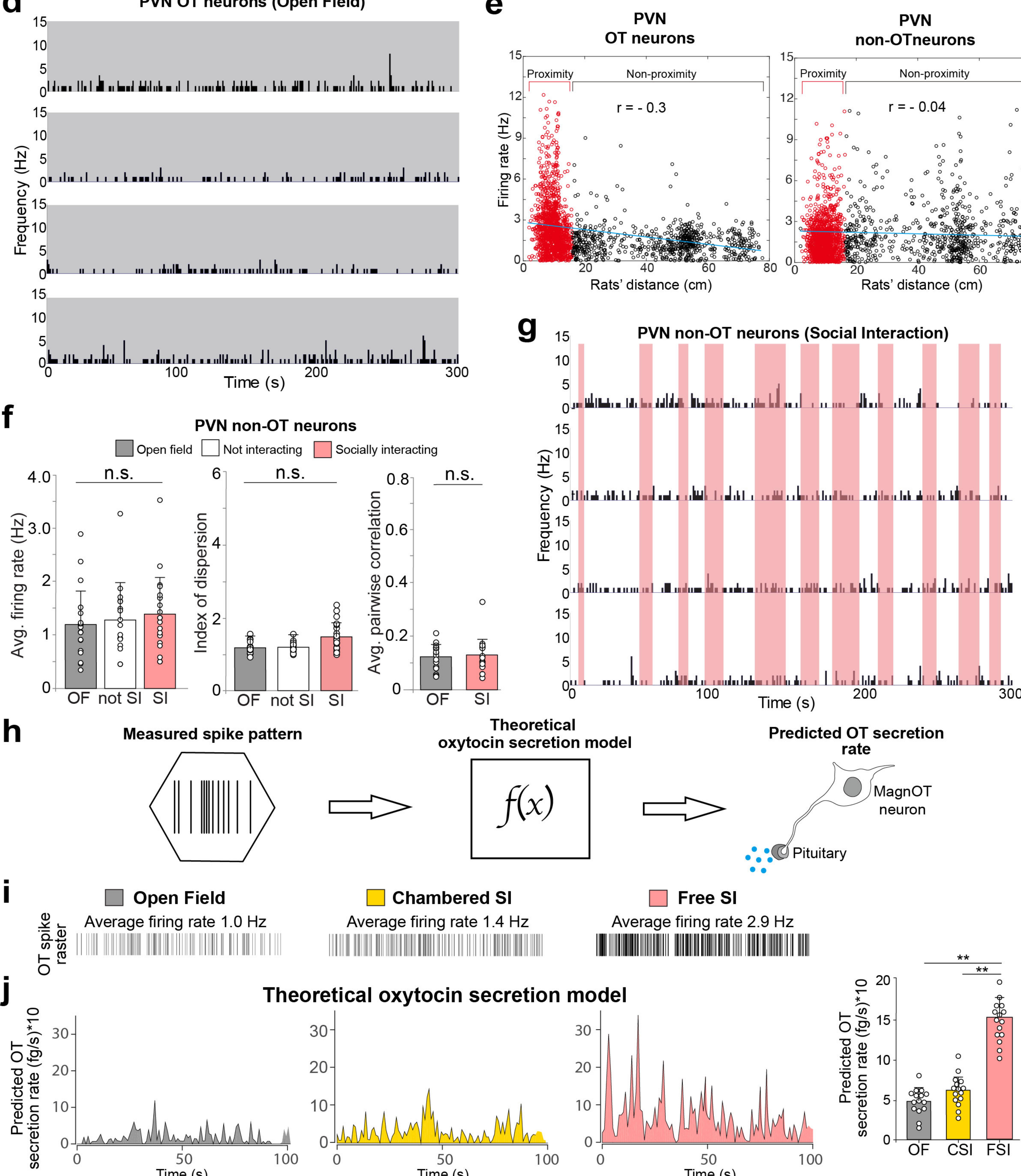


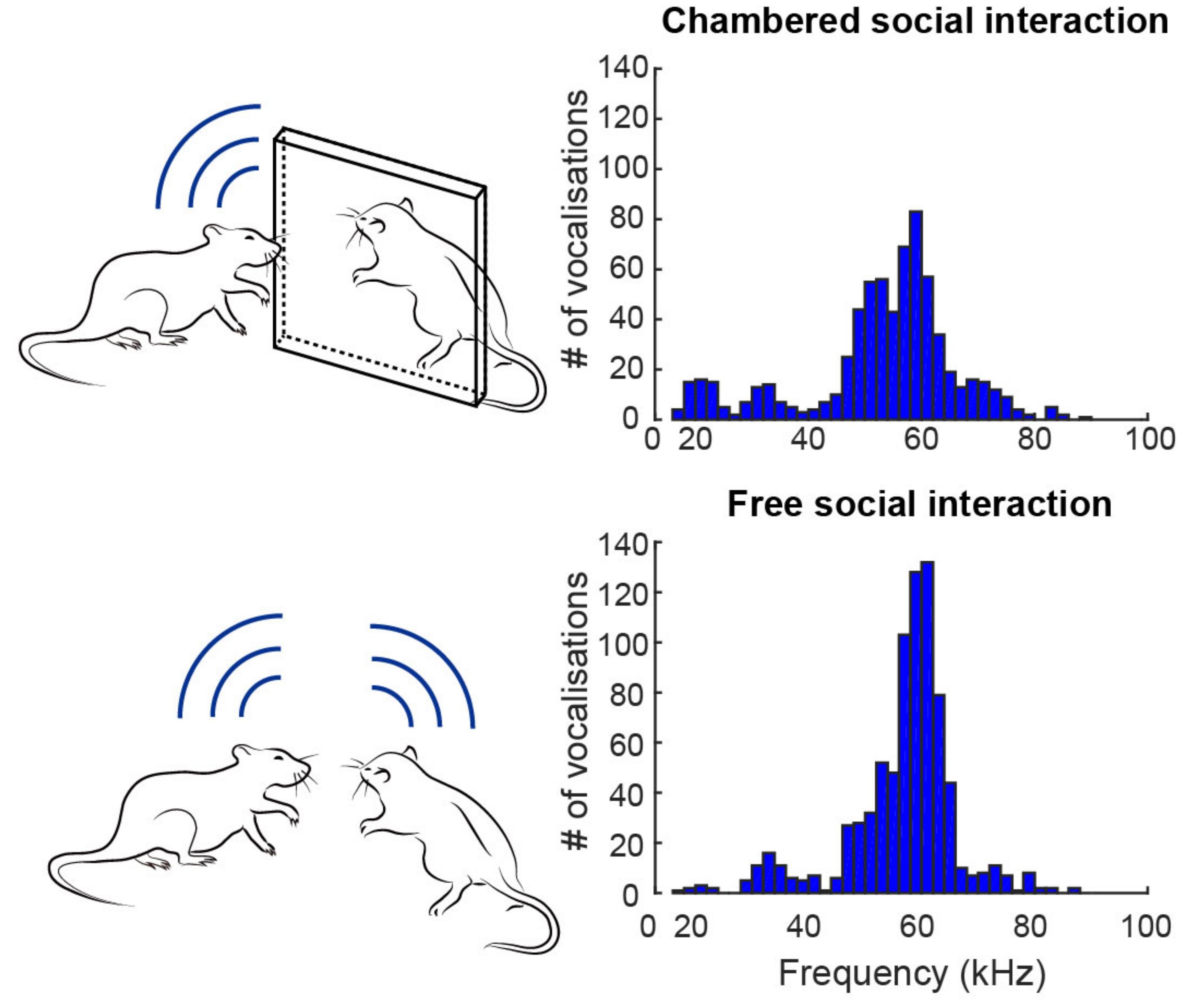

Trills

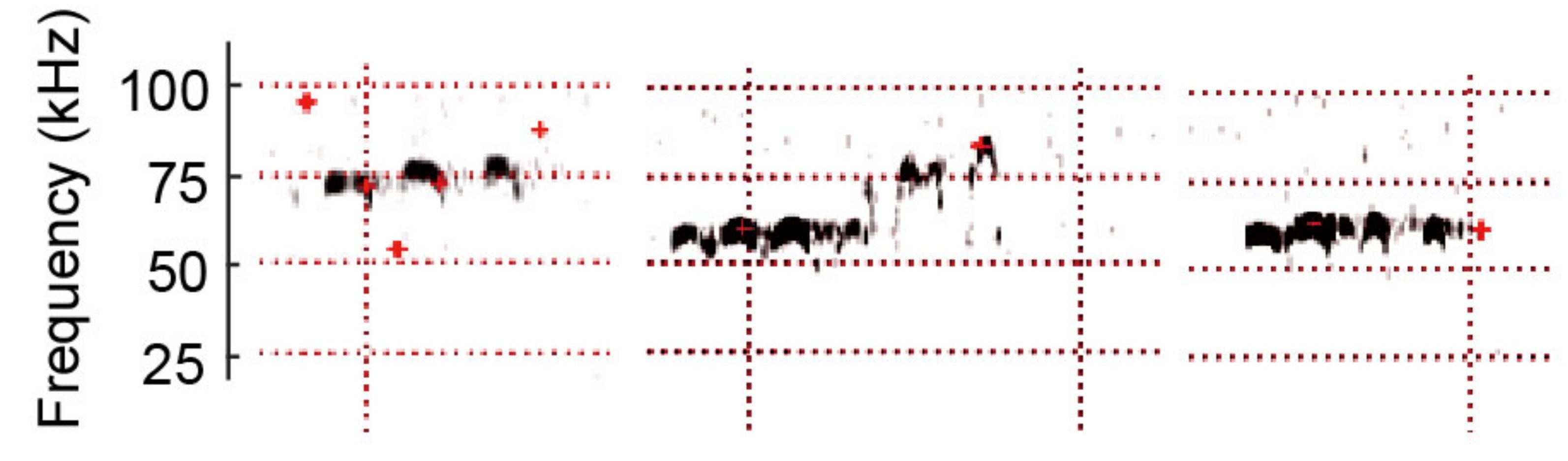

Calls
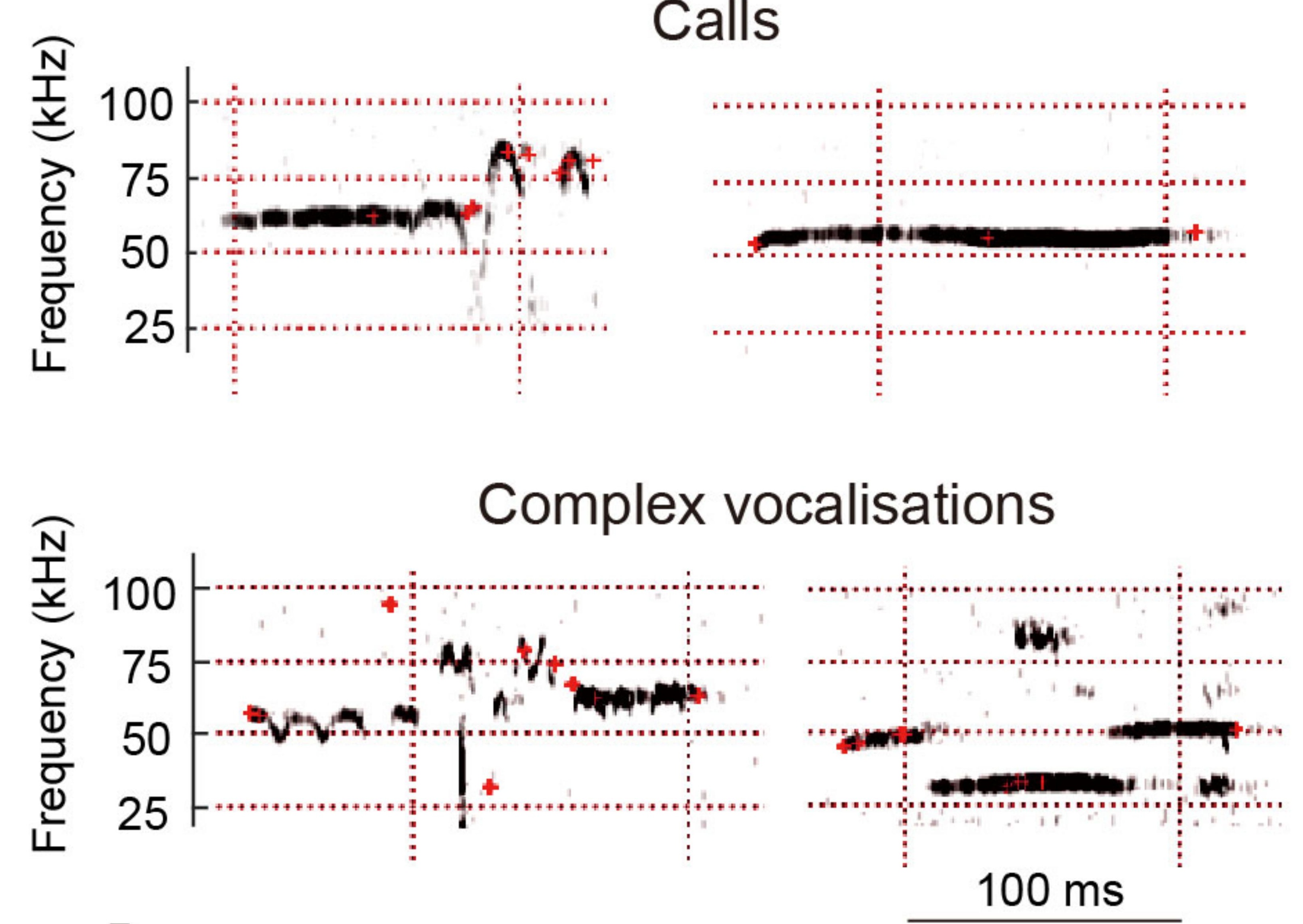

C
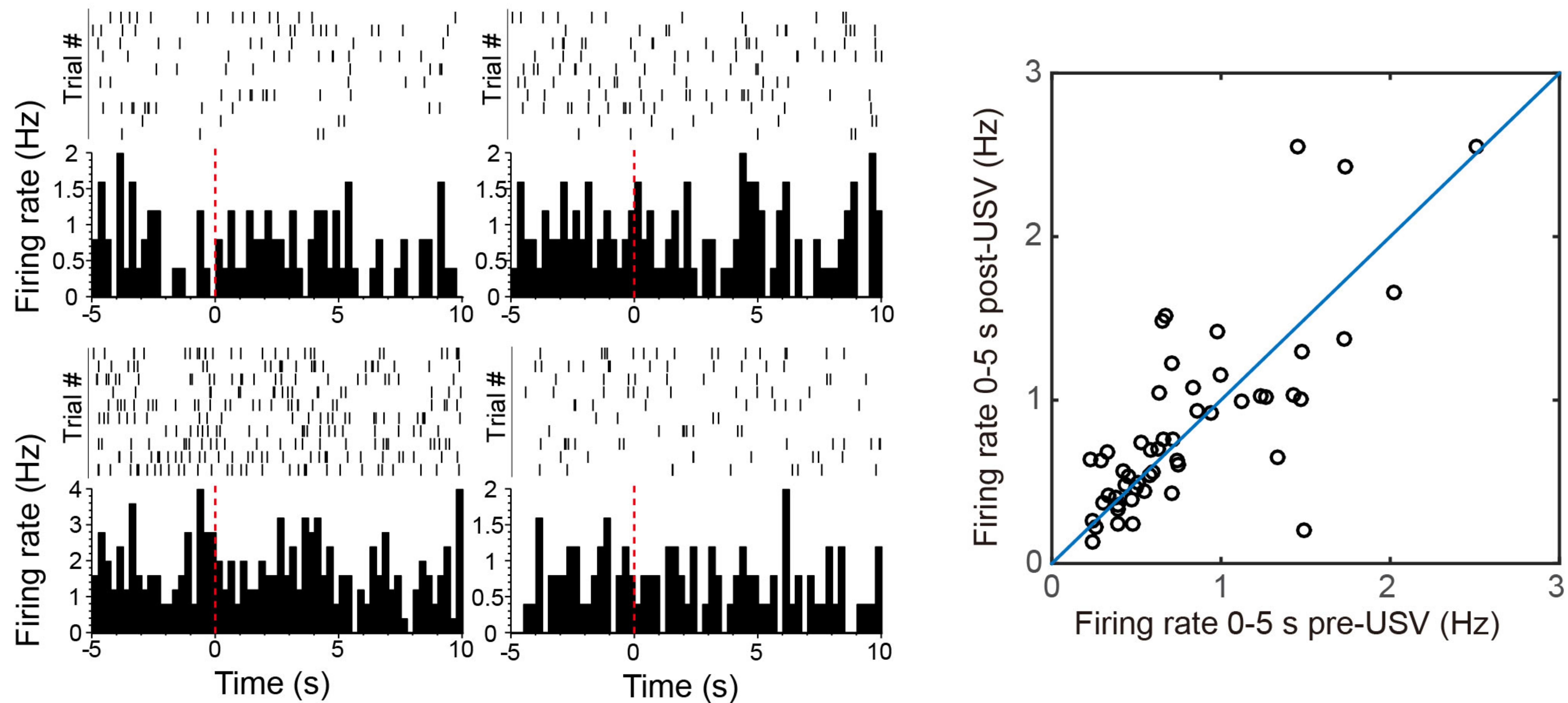

e

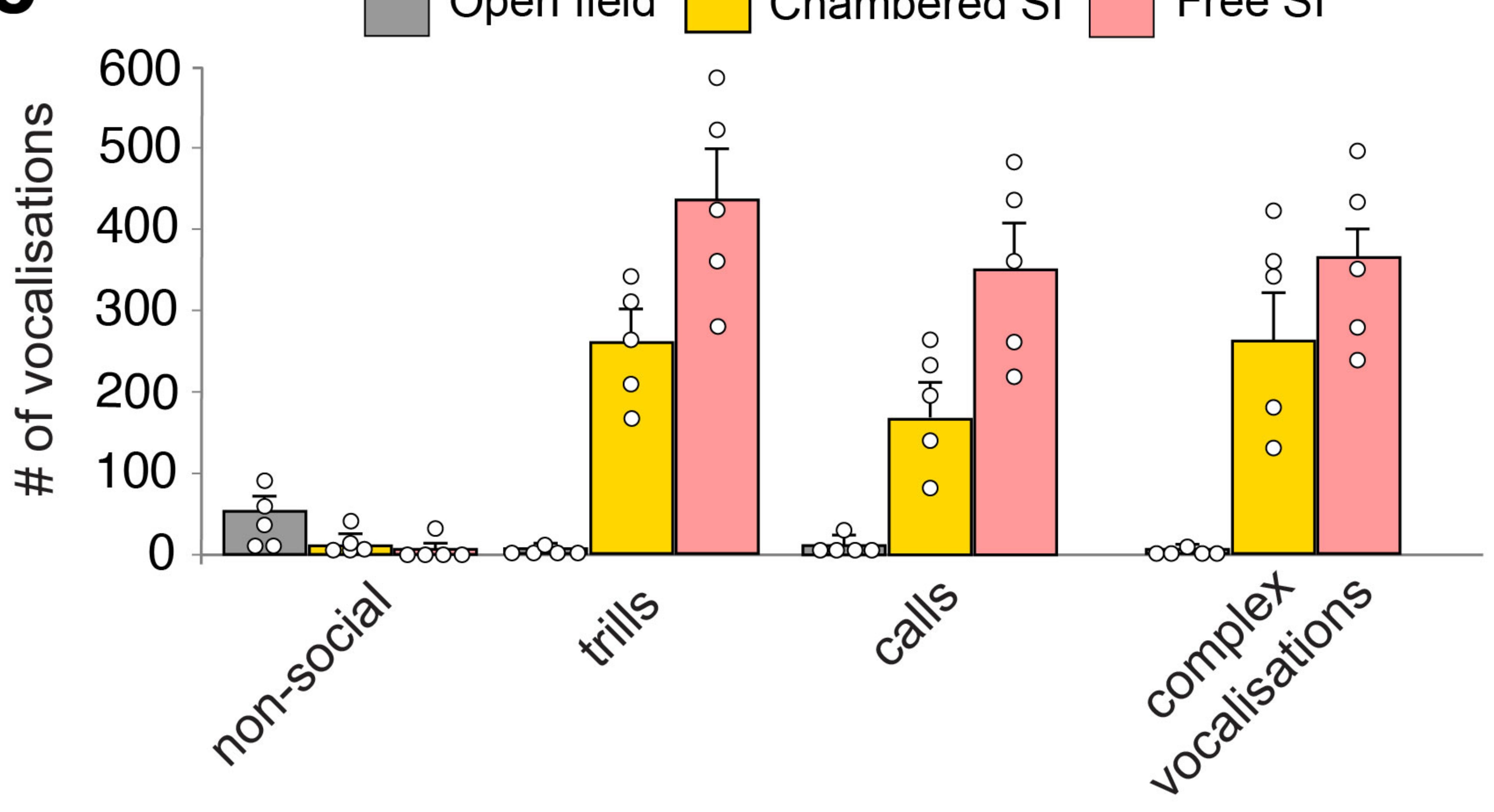


a

b
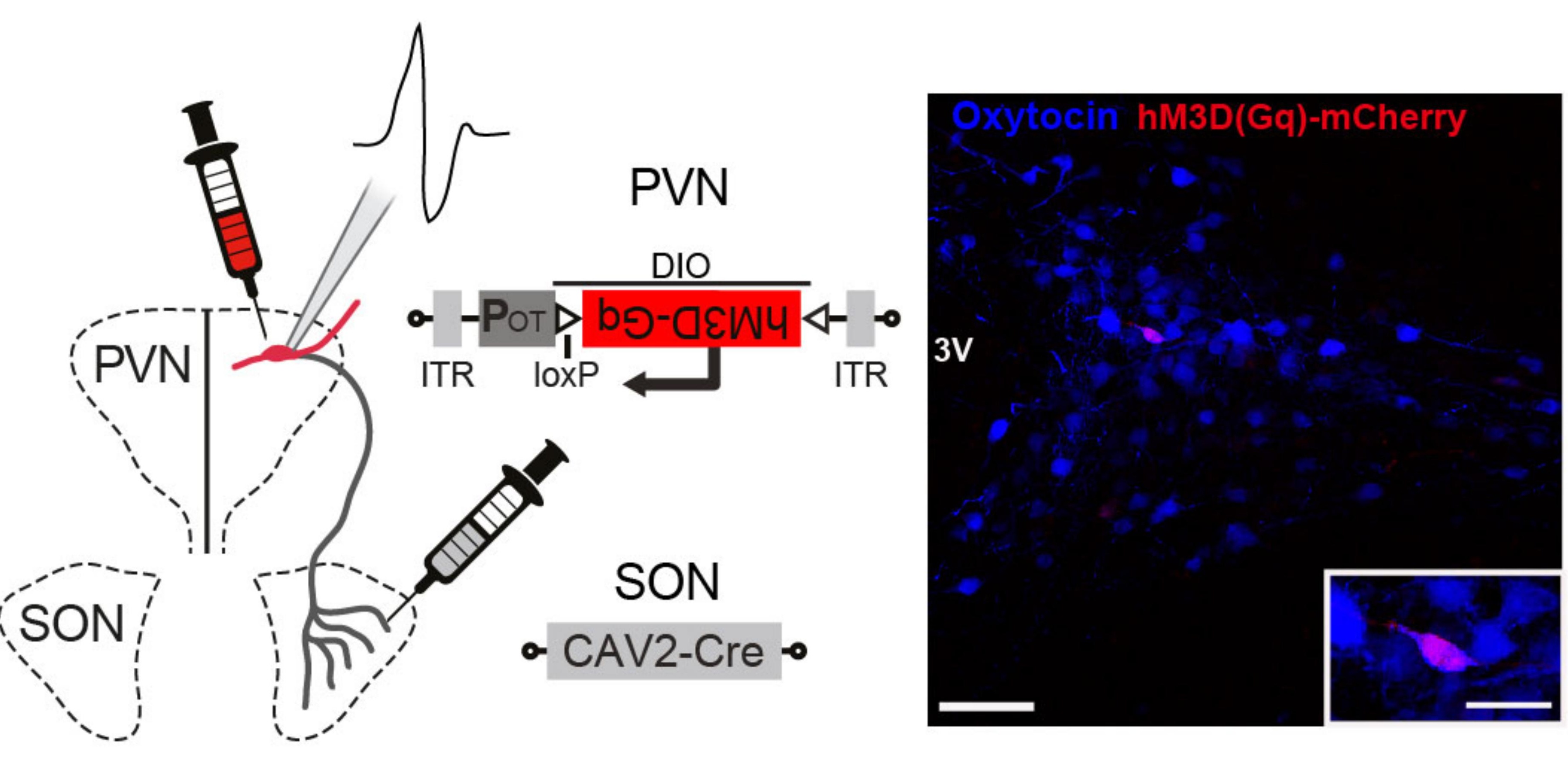

d
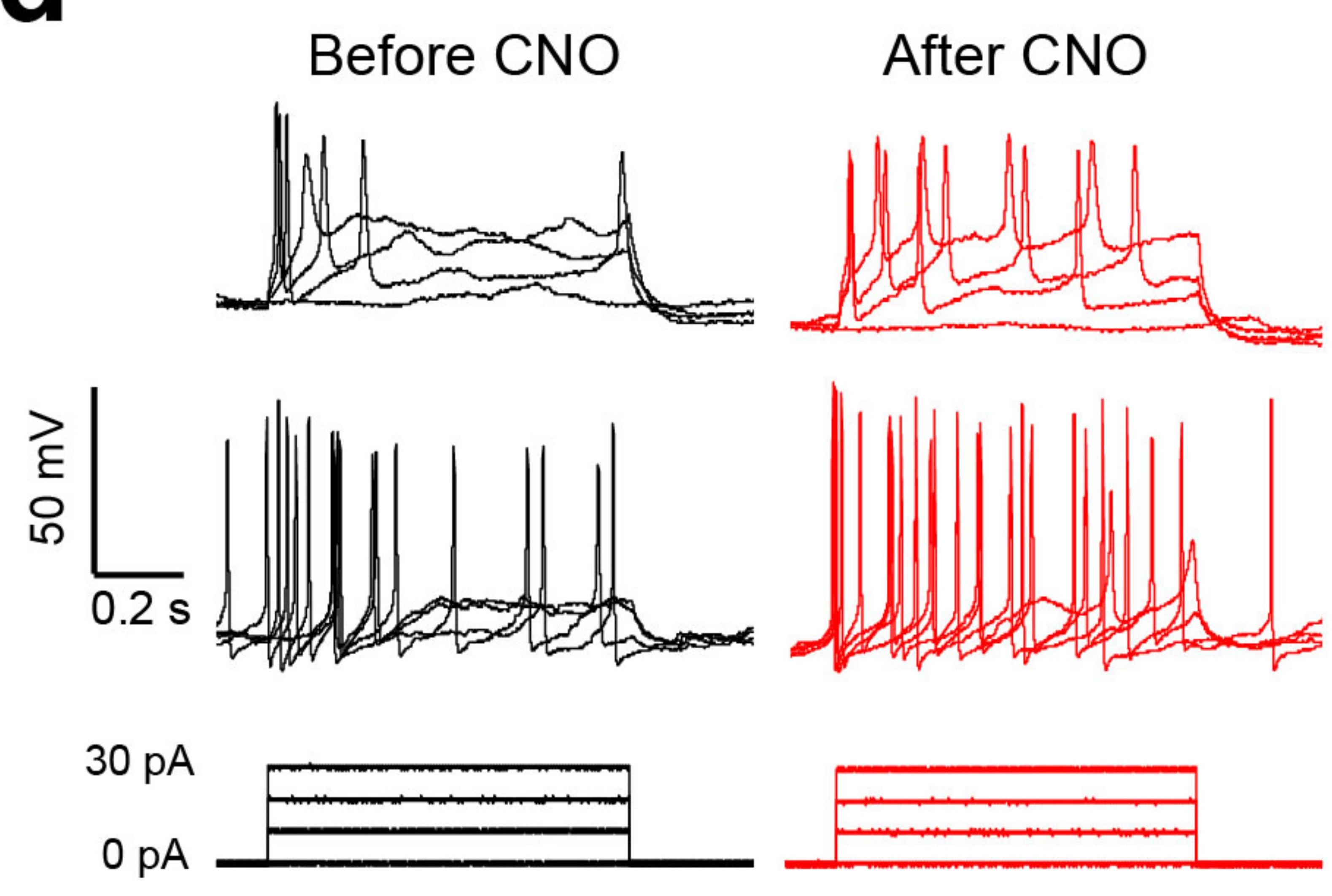

g
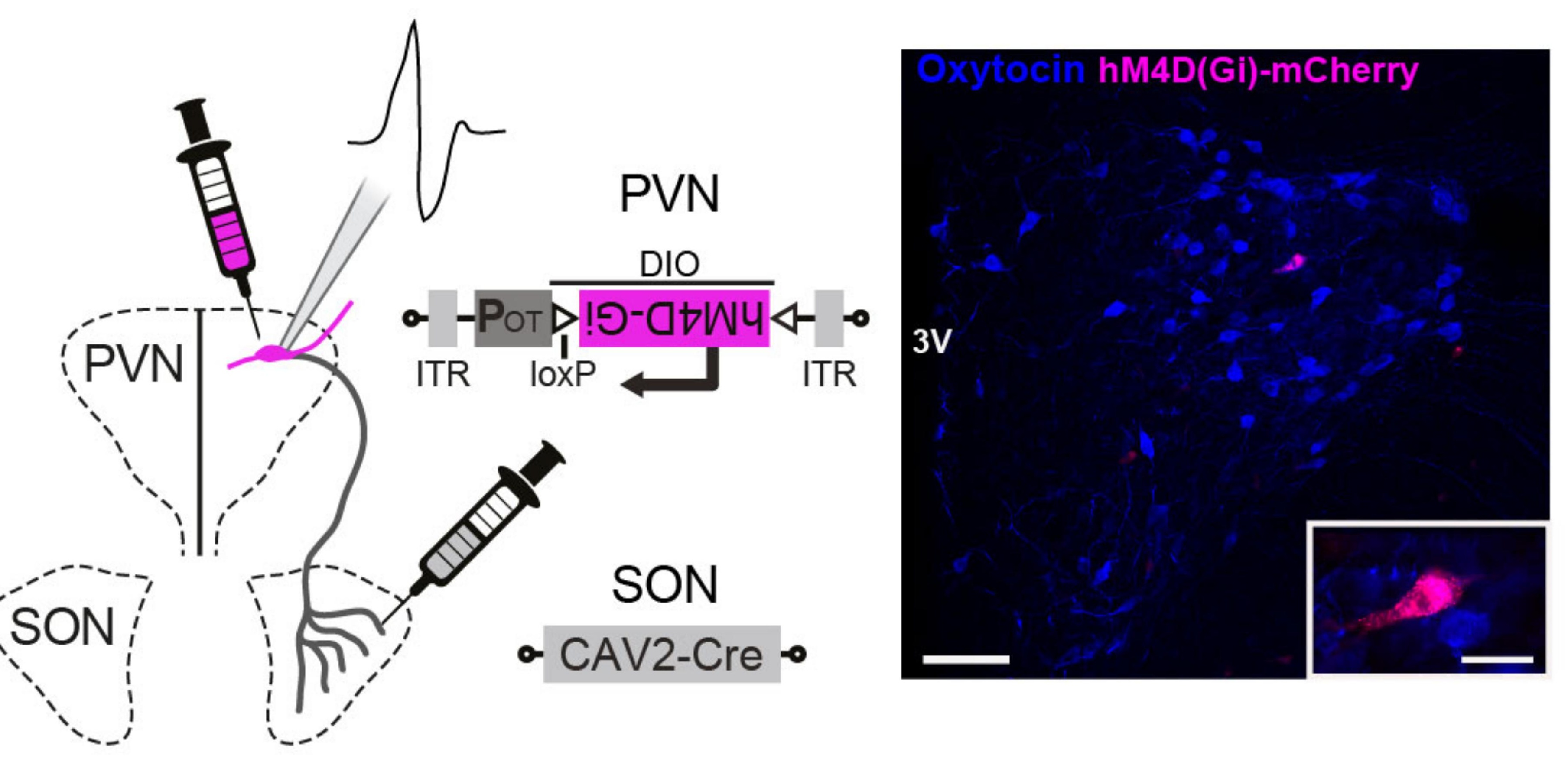

j

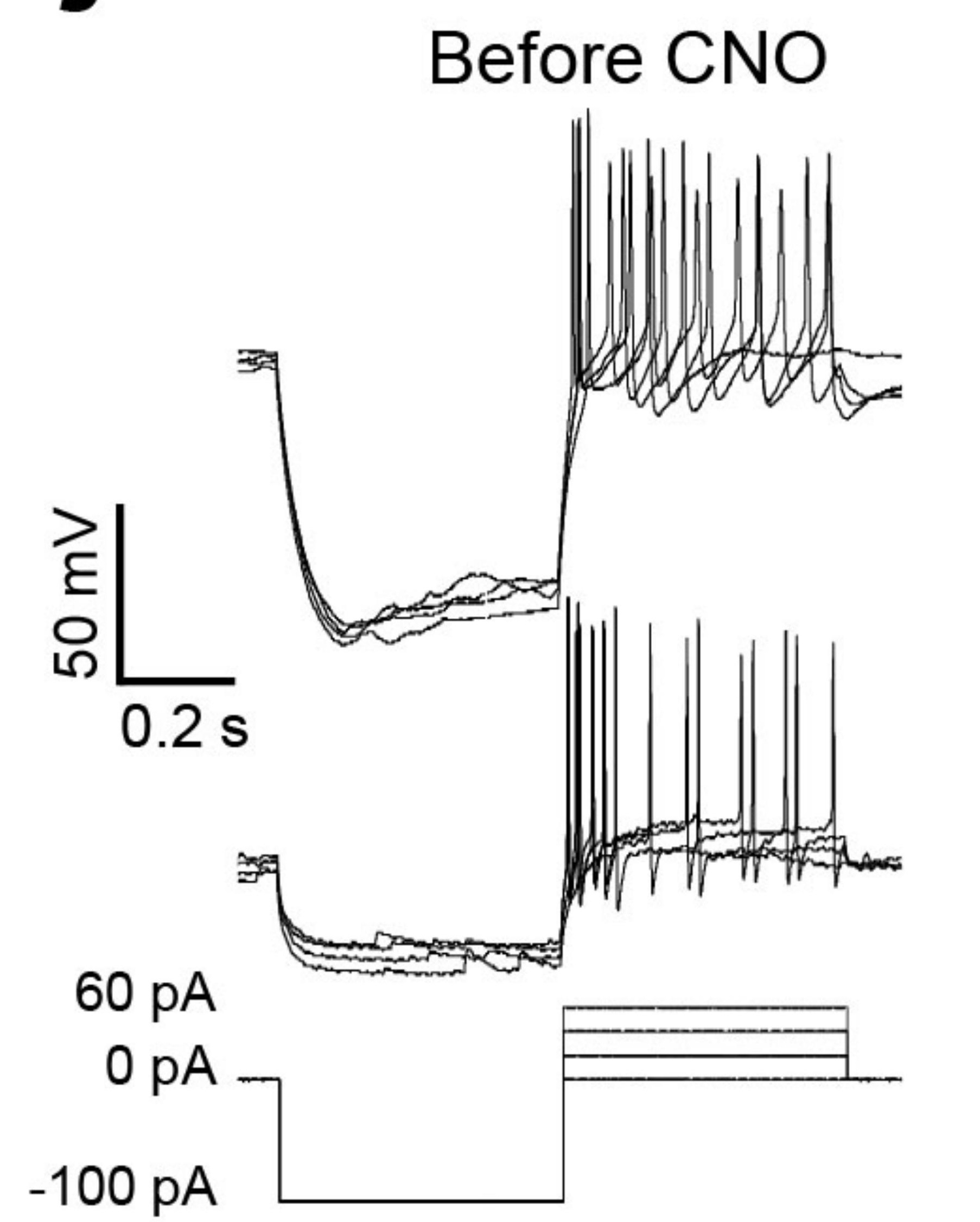

Before CNO

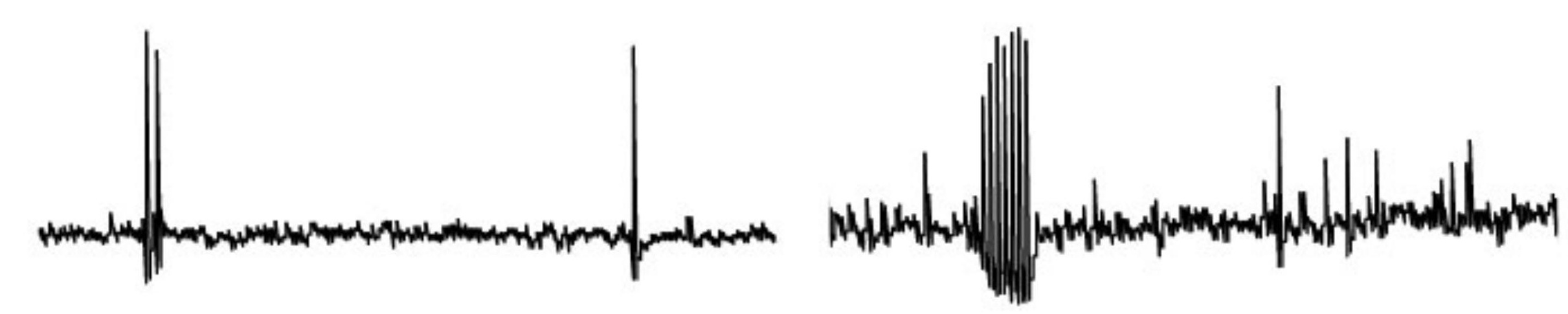

After CNO

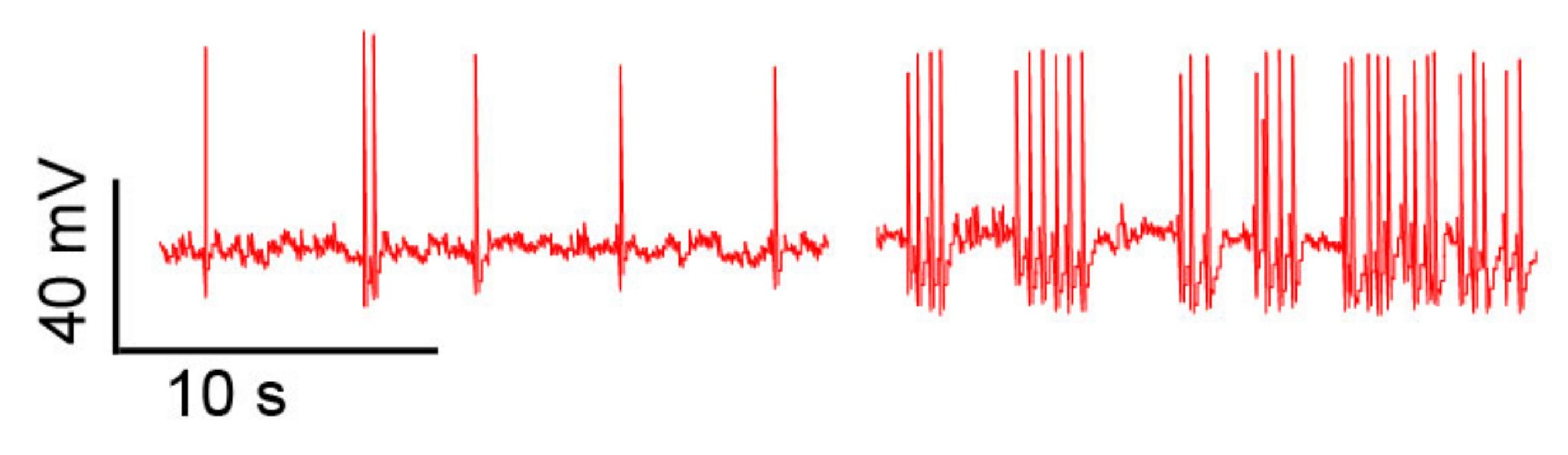

e

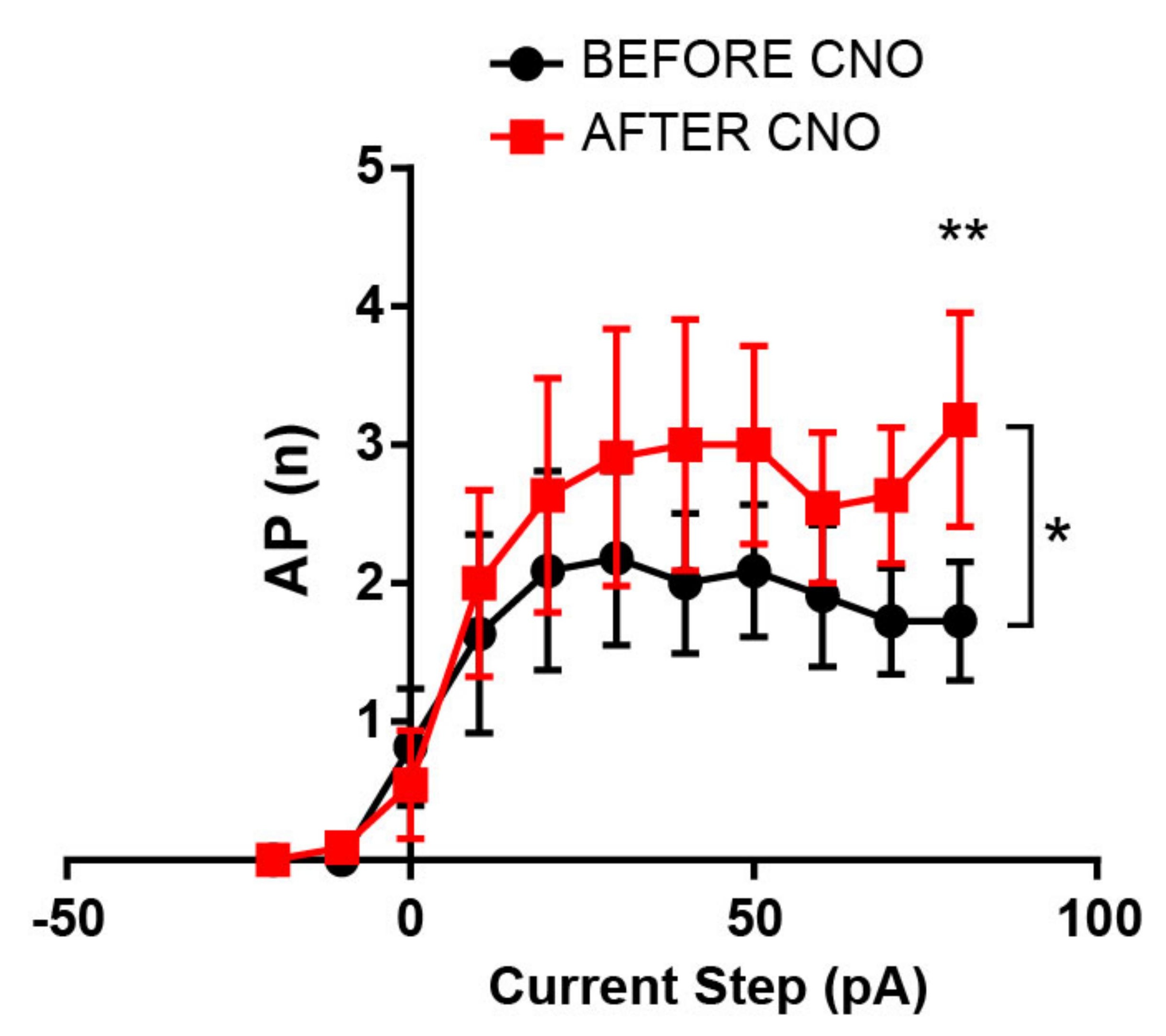

h

Before CNO

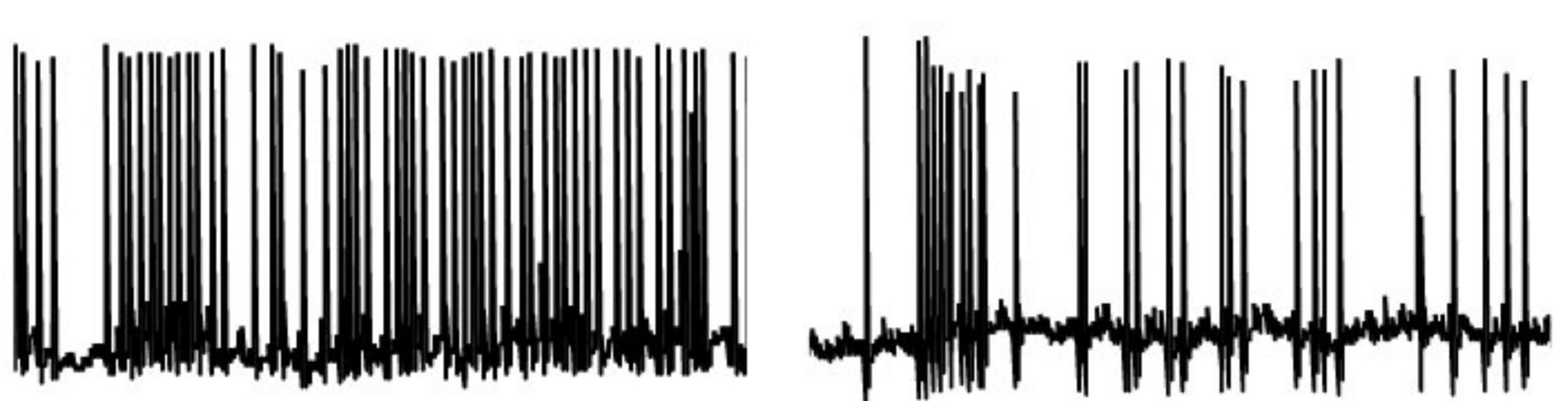

After CNO

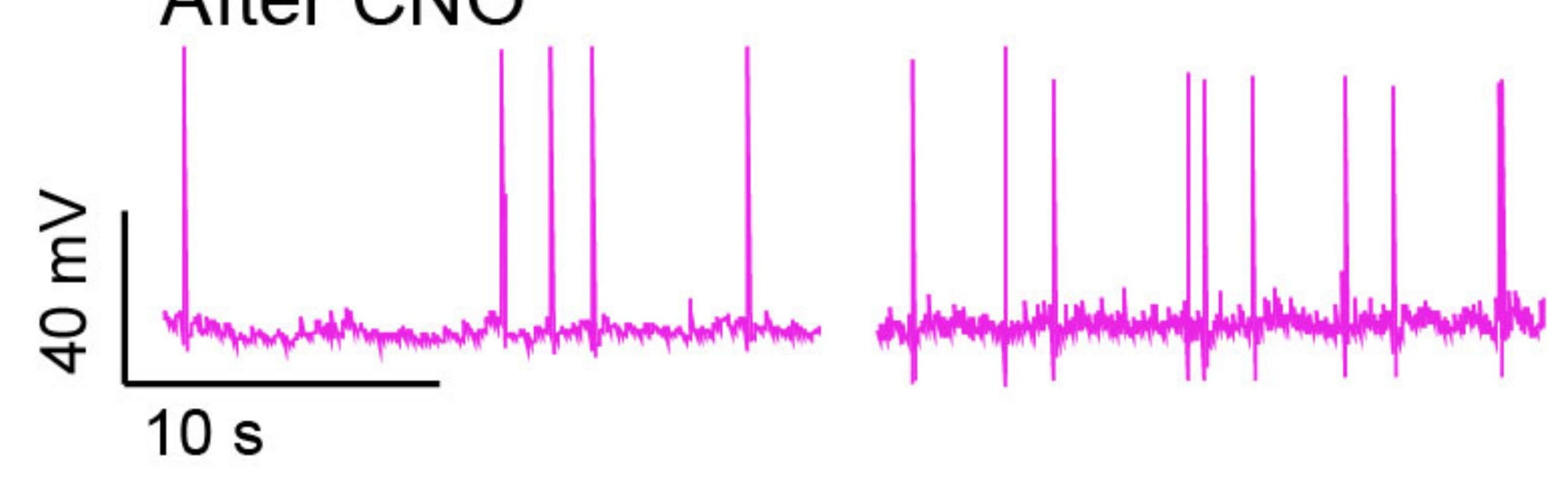

k

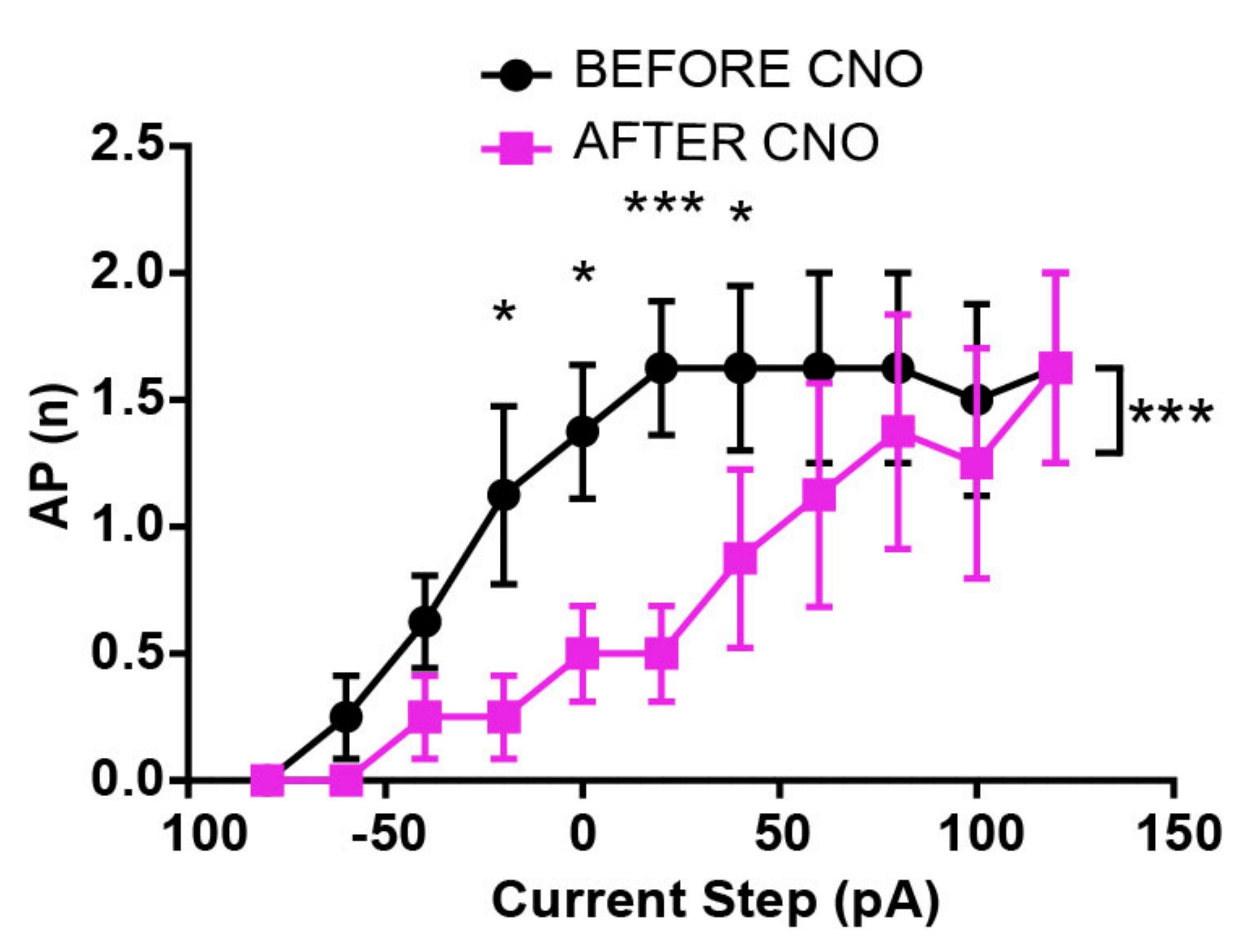

C

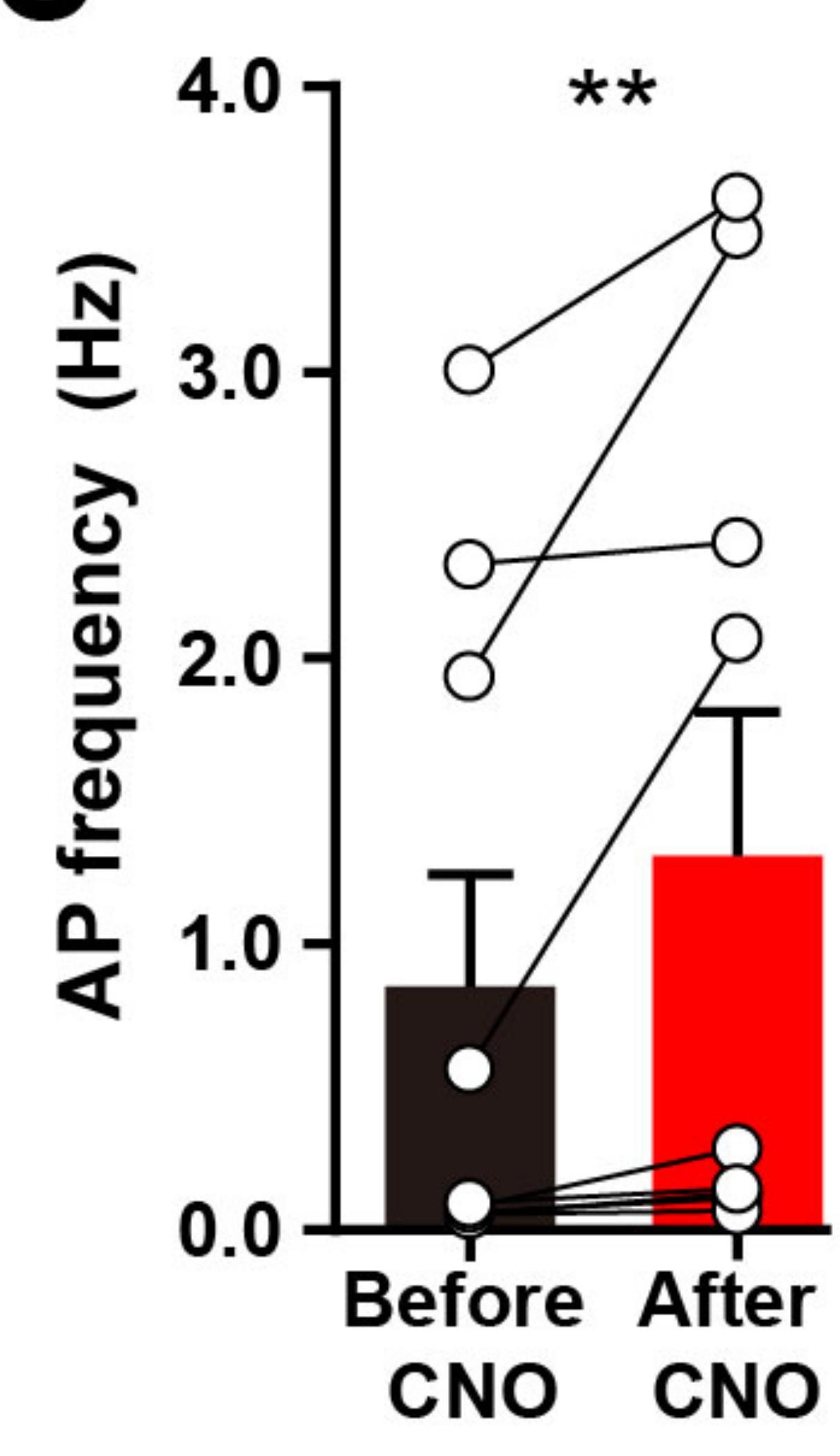

f

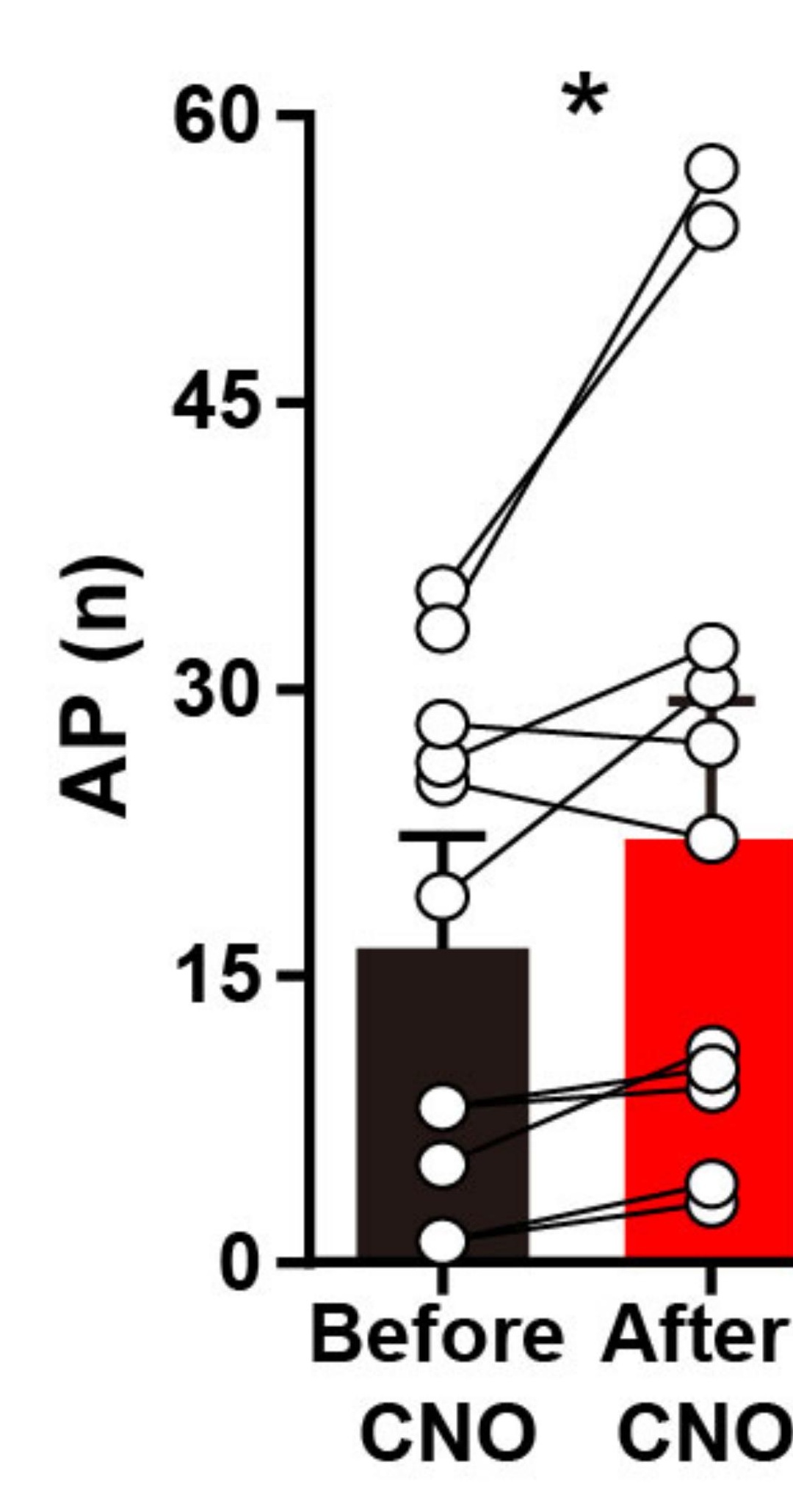

9
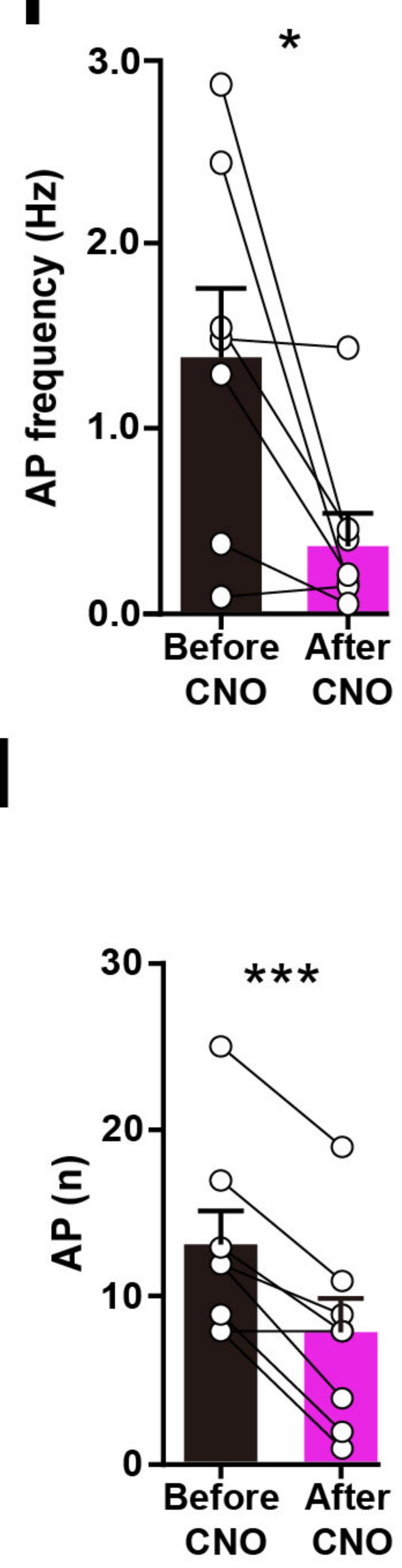

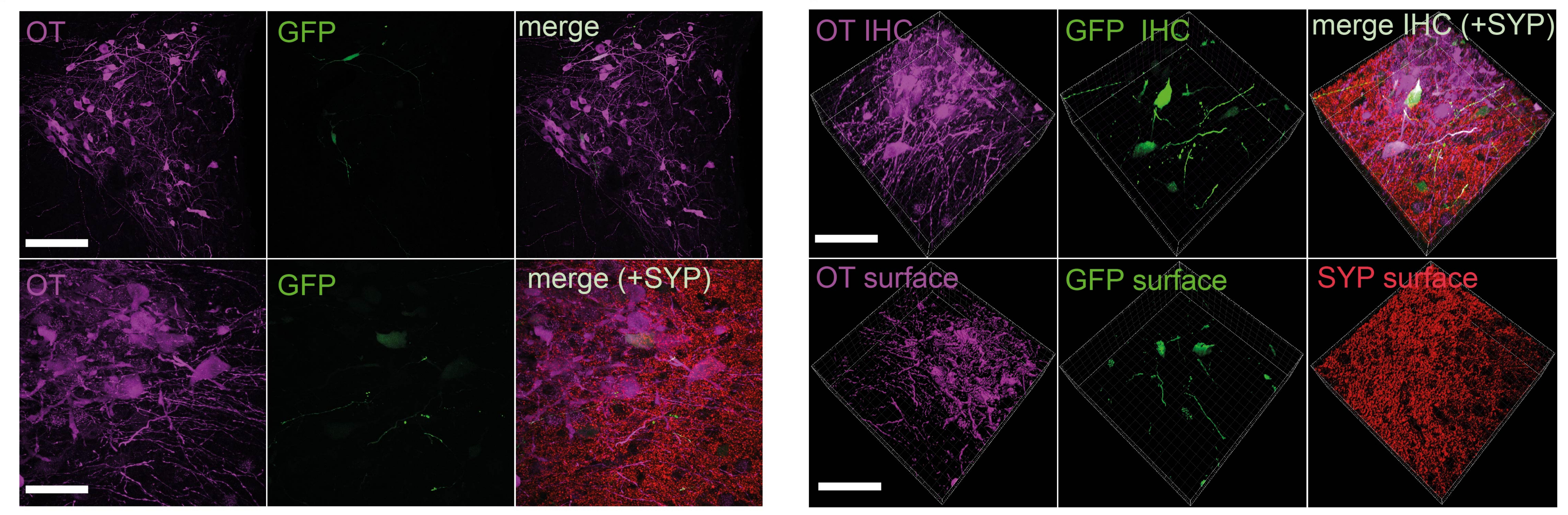

\section{C}

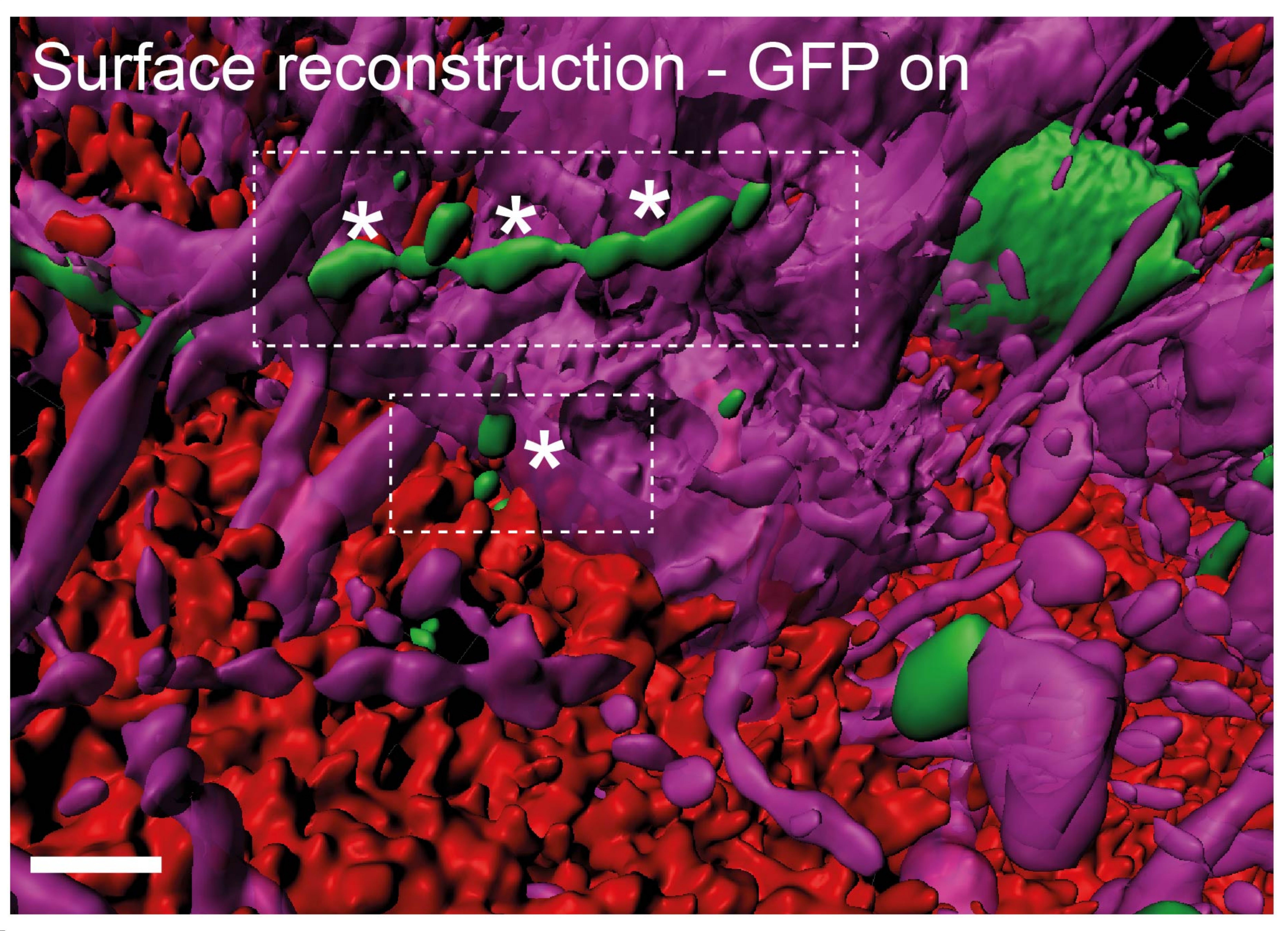
d
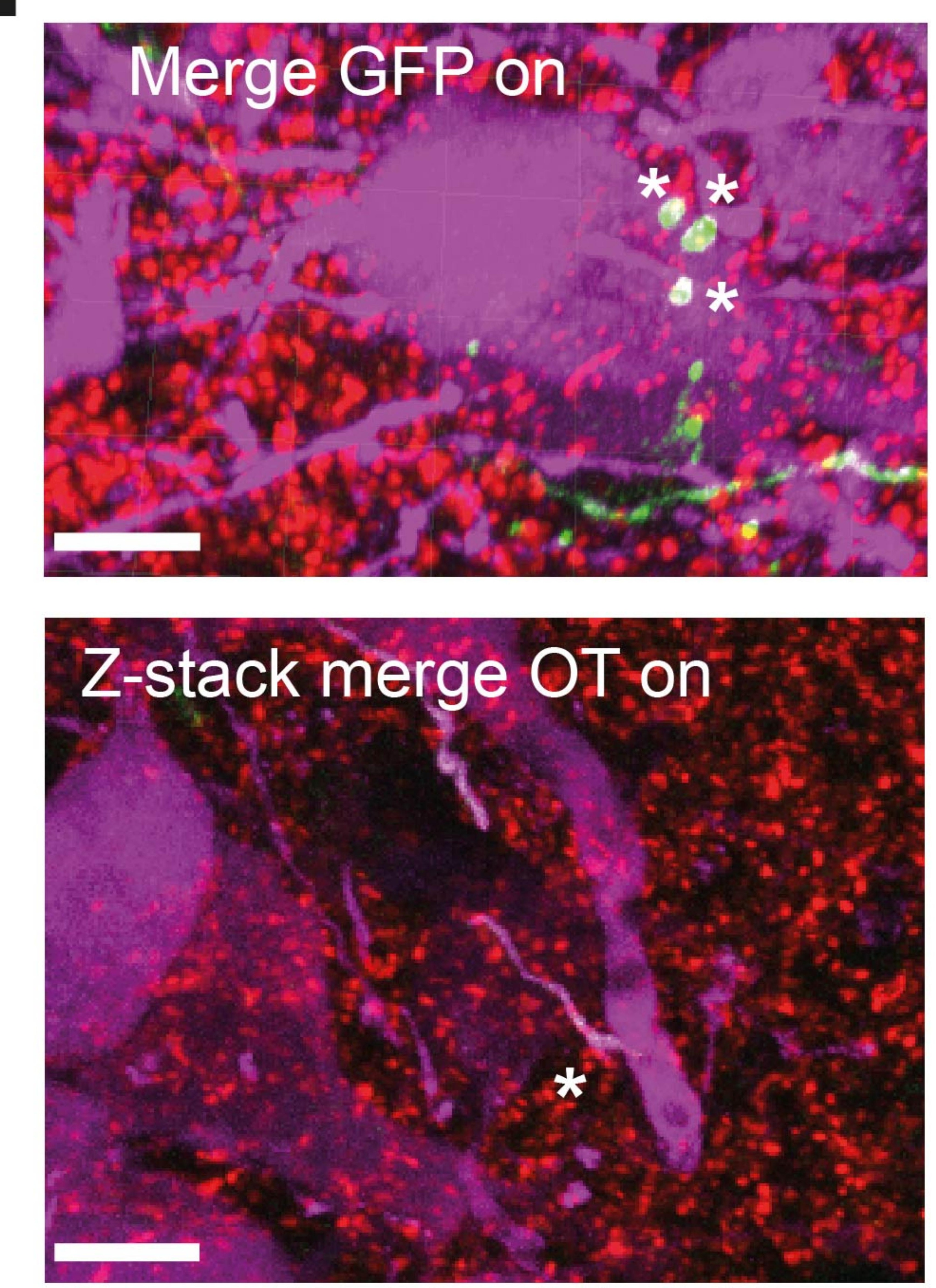

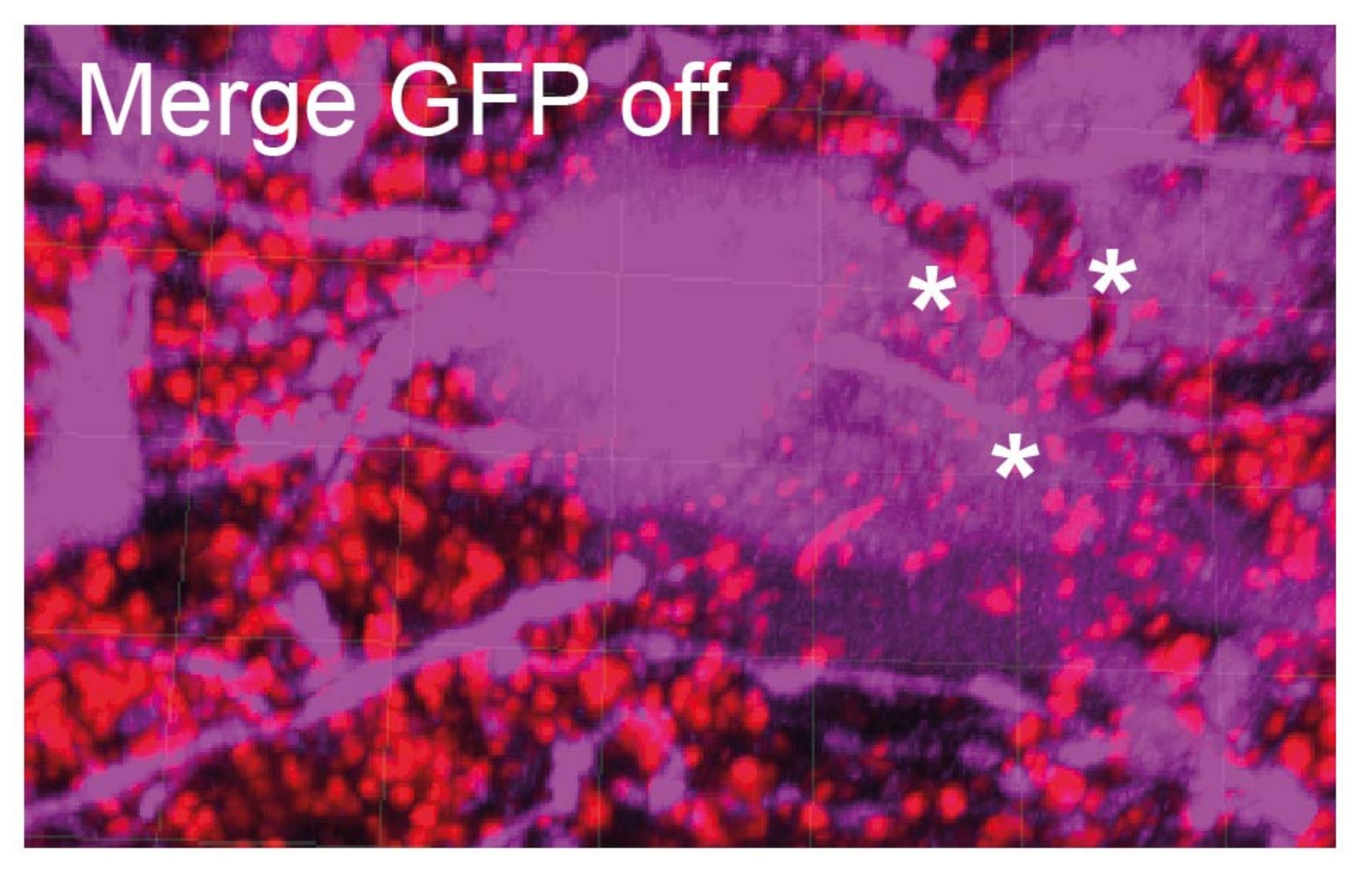

Z-stack merge OT off

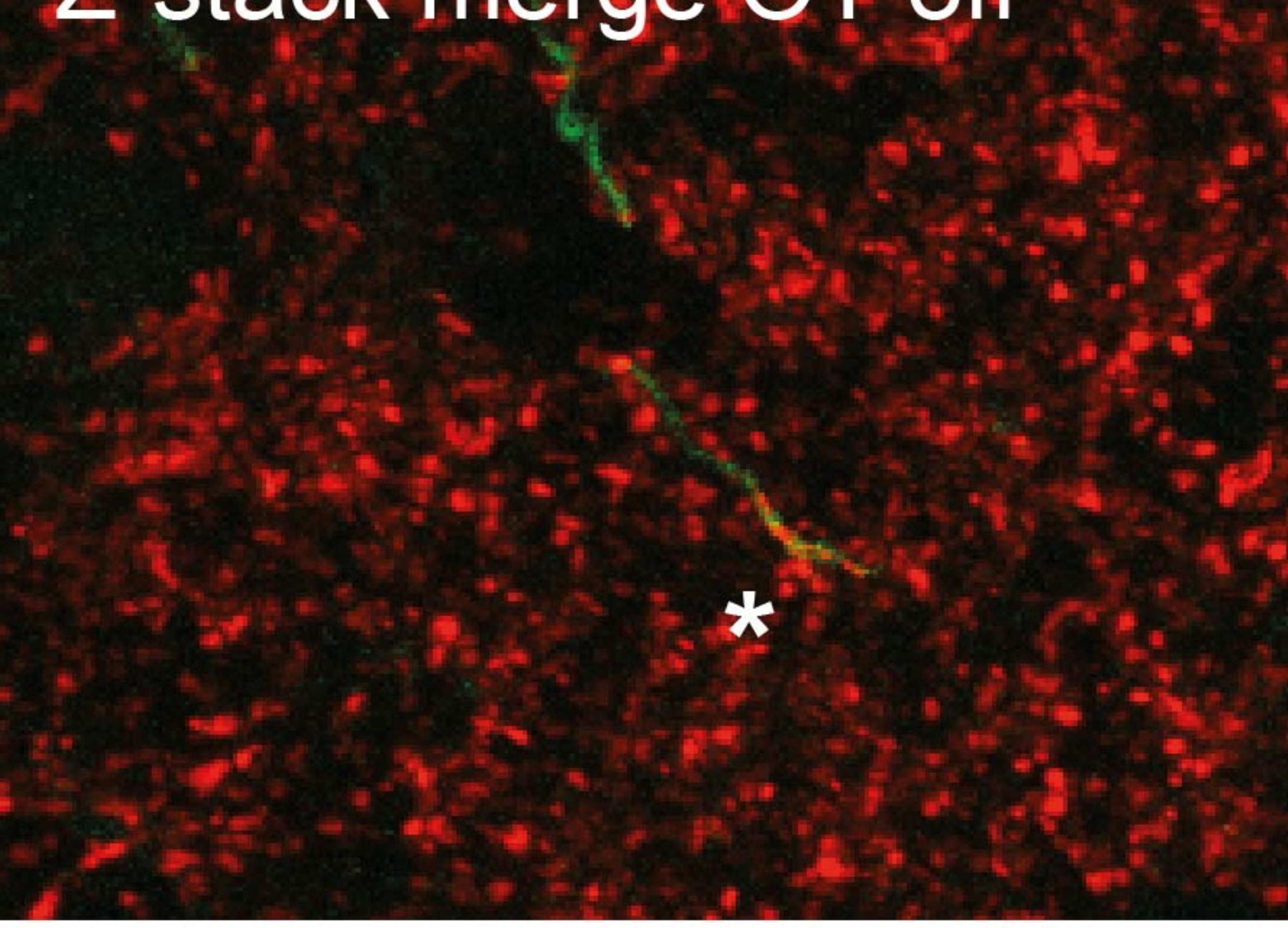

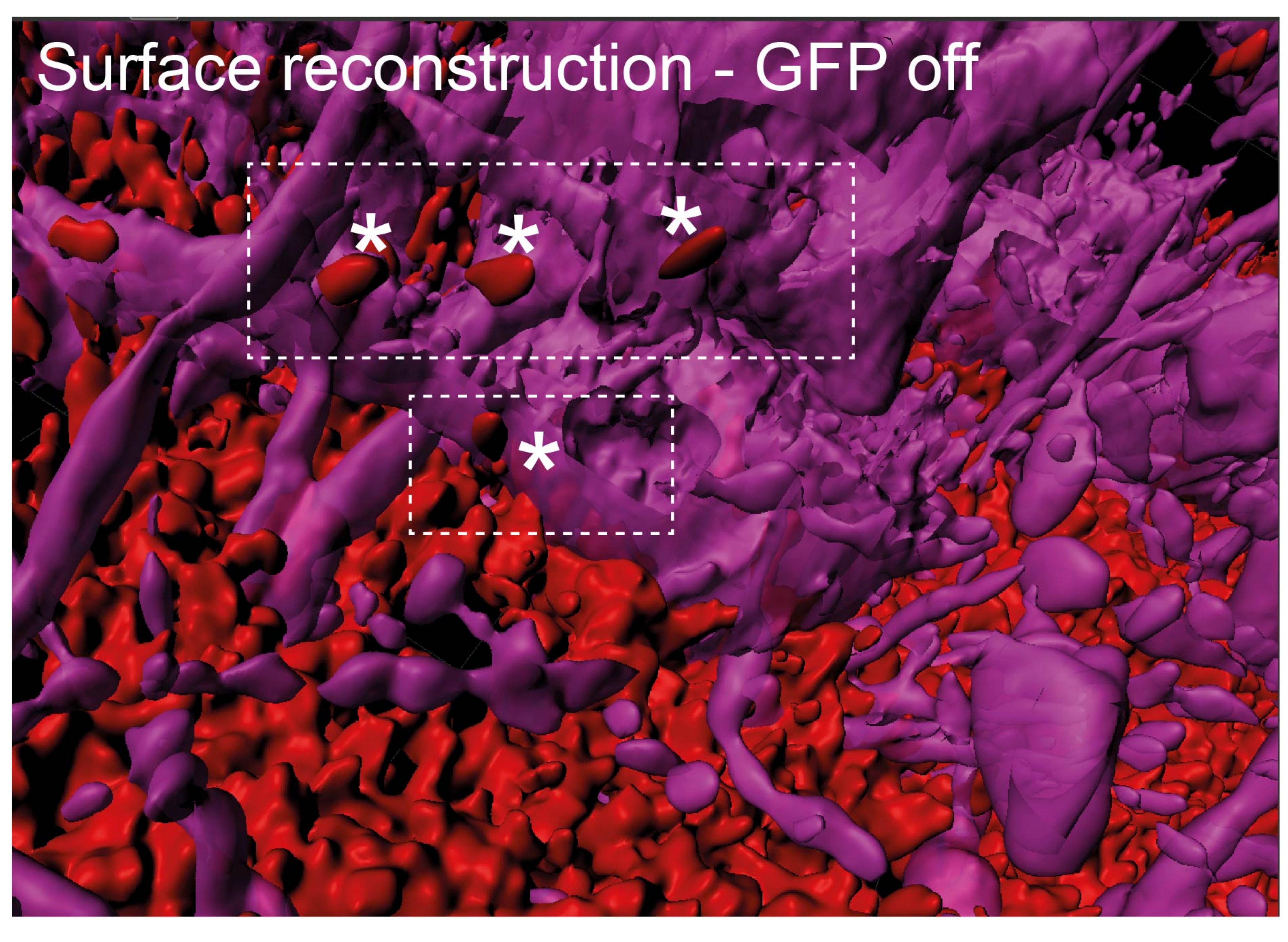

e

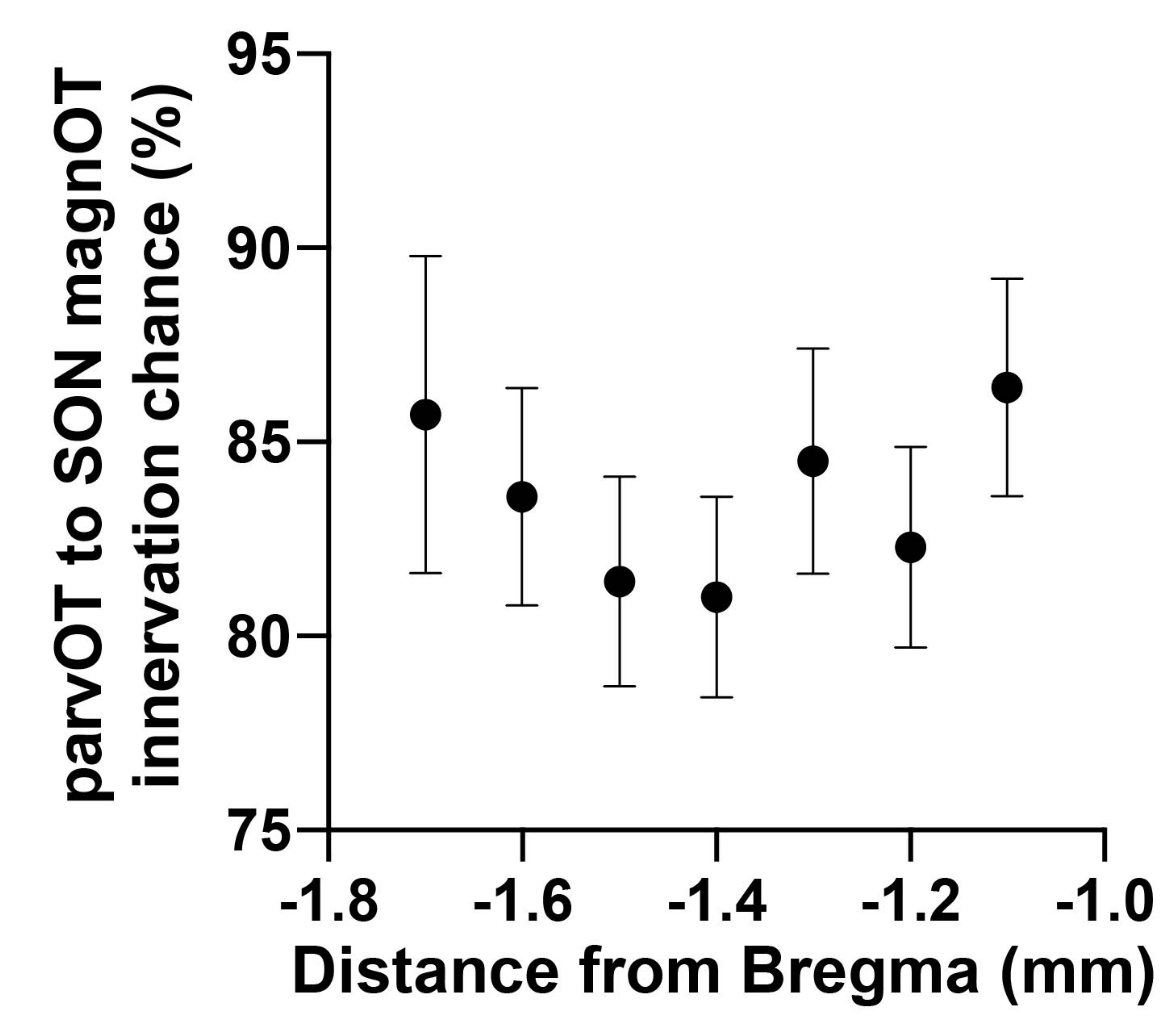




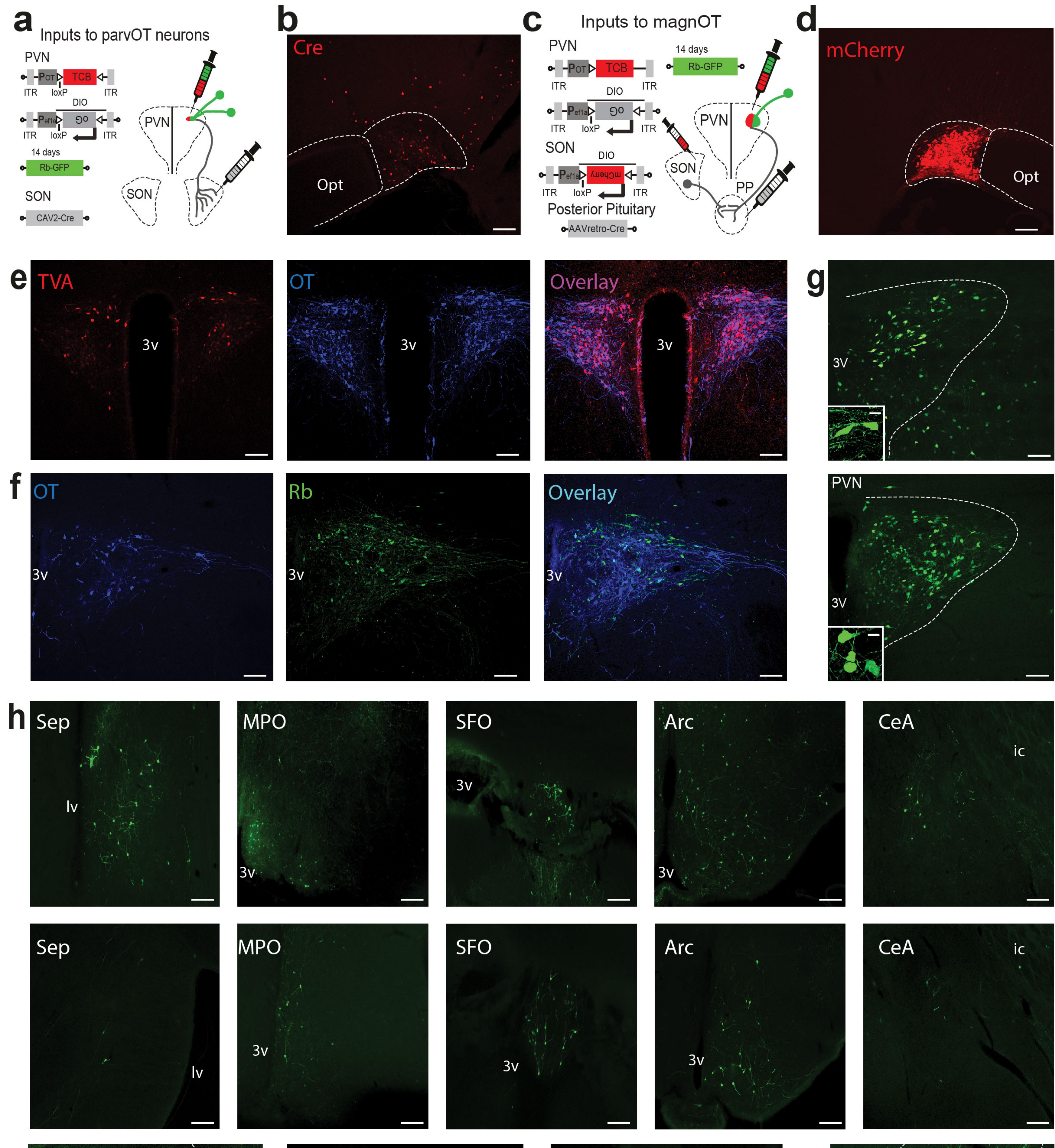

\author{
PV
}

a-PotP TCB «-

ITR IOXP ITR

- -Pef1a 504

ITR loxP

Rb-GFP

SON

\section{SON}

C Inputs to magnOT

Overlay

$\mathrm{Hb}$

j $\mathrm{SN}$ i Ins

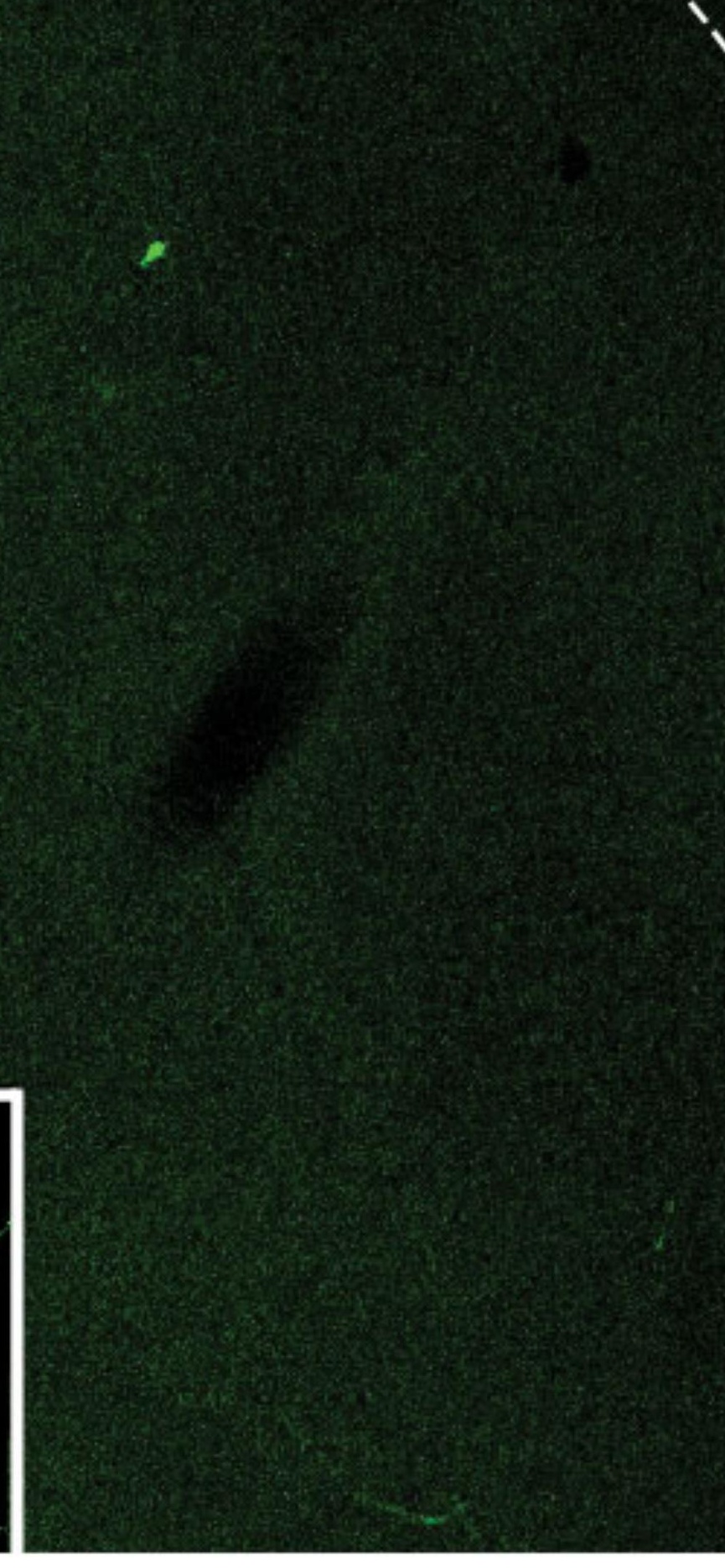

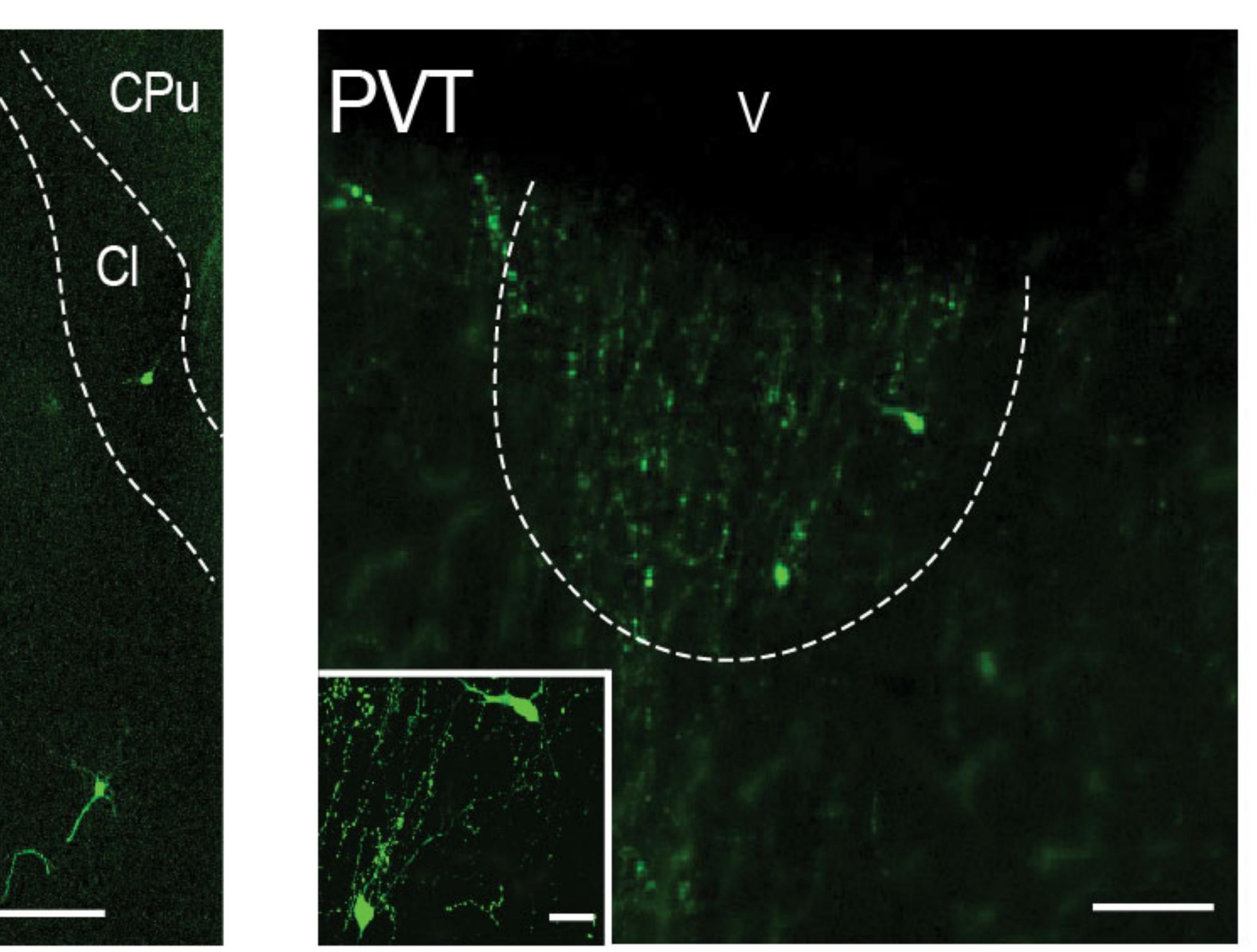

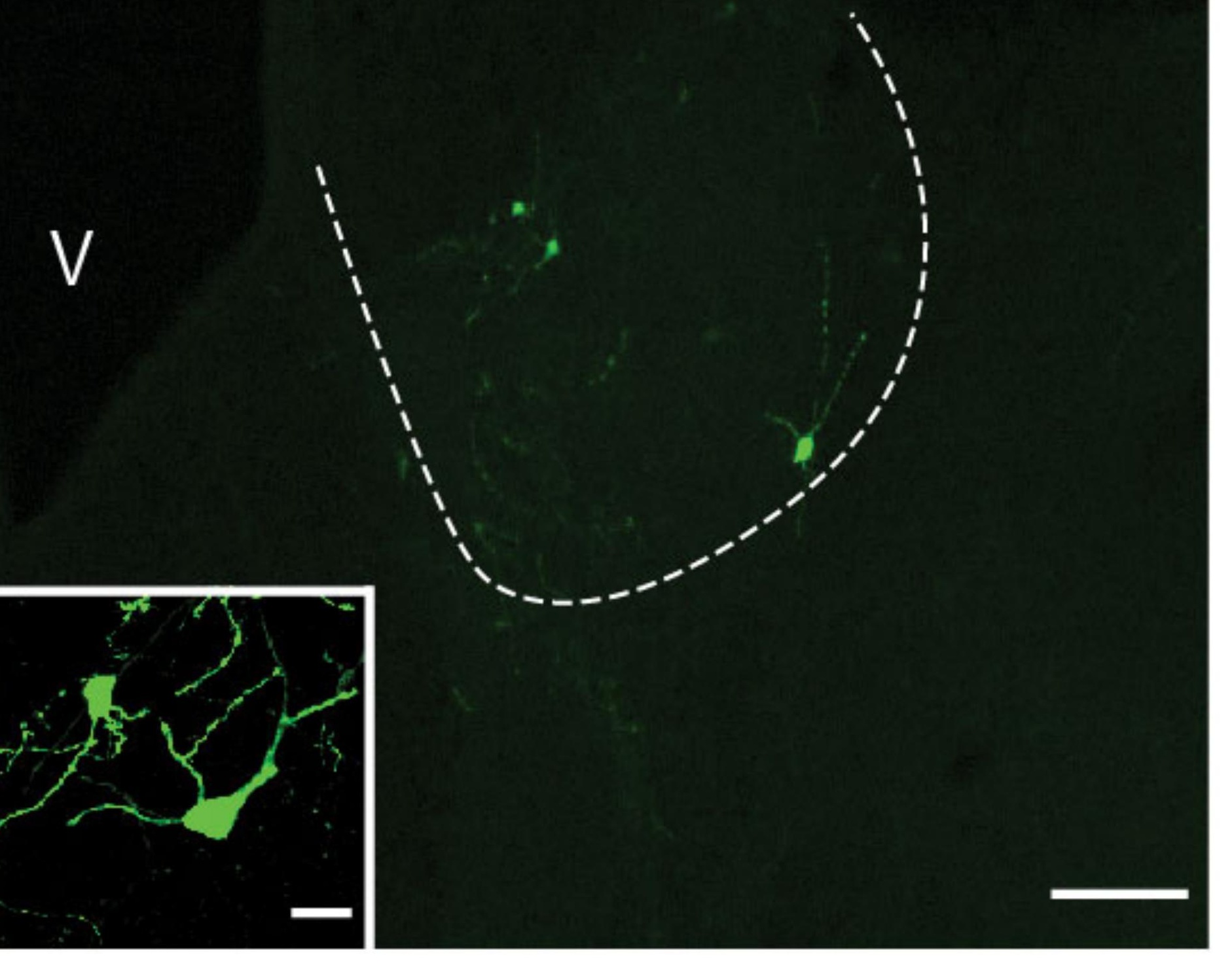



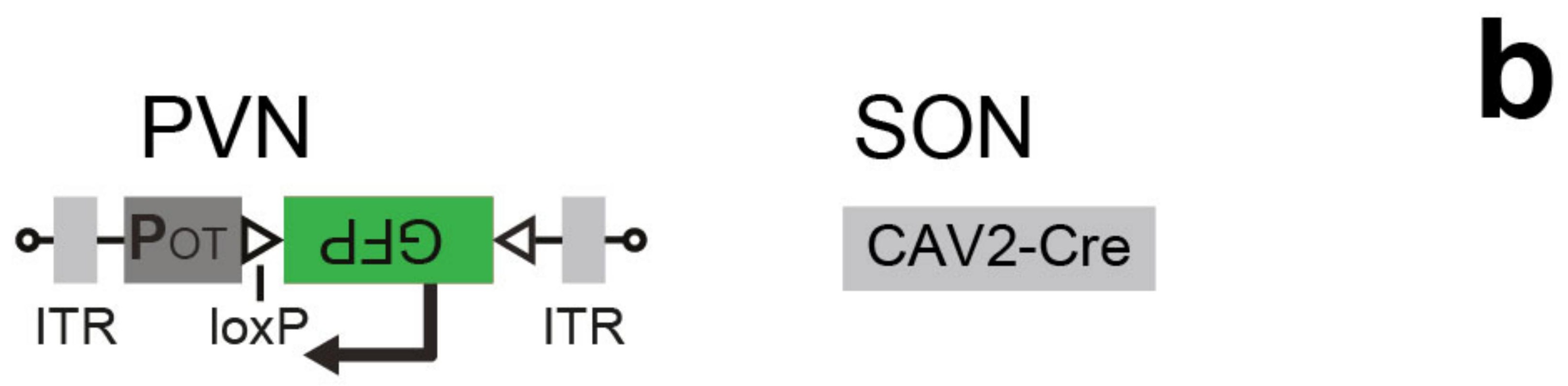

CAV2-Cre

\section{Chambered SI}

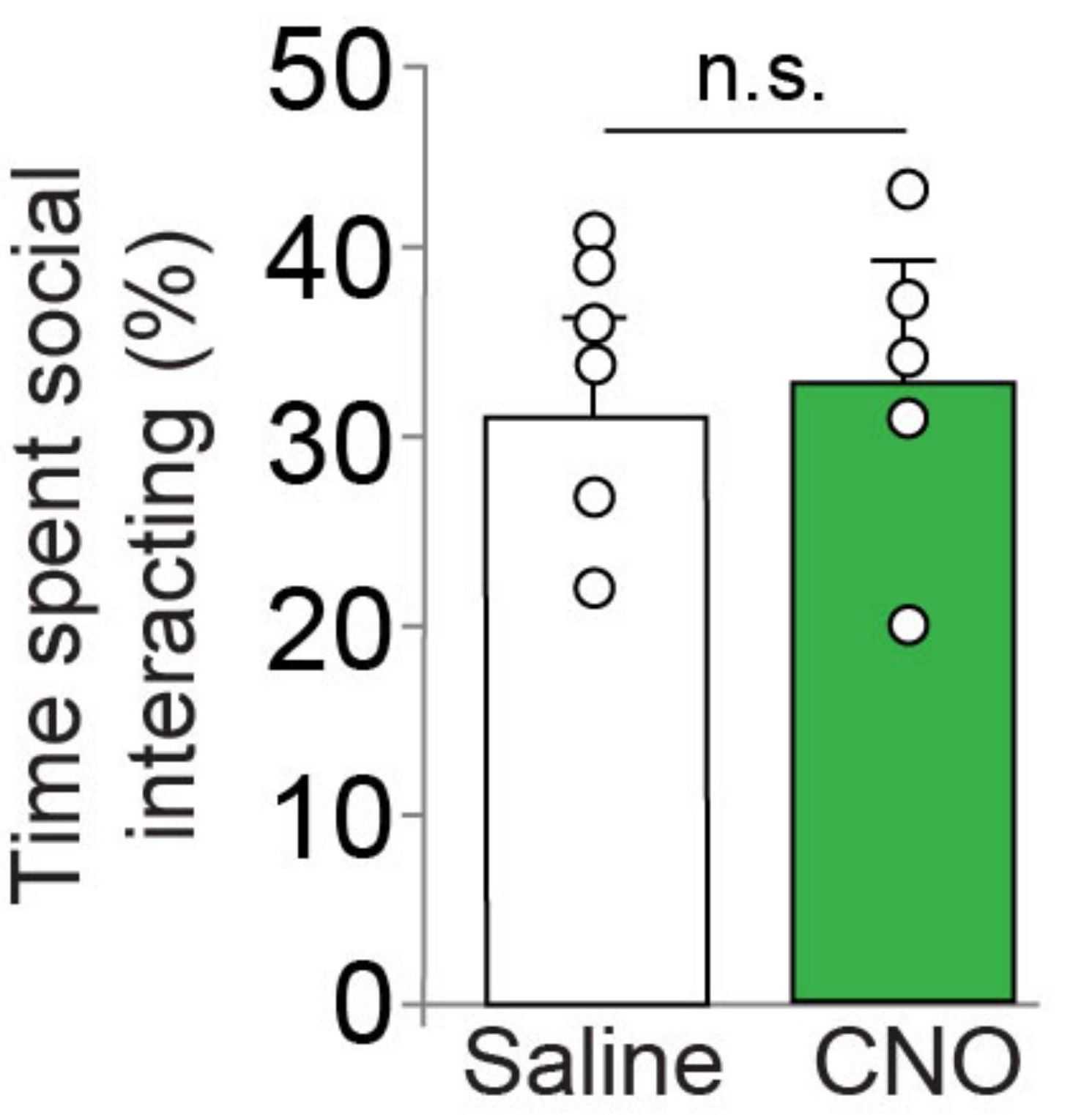

d

Locomotor activity

3.0 n.s.

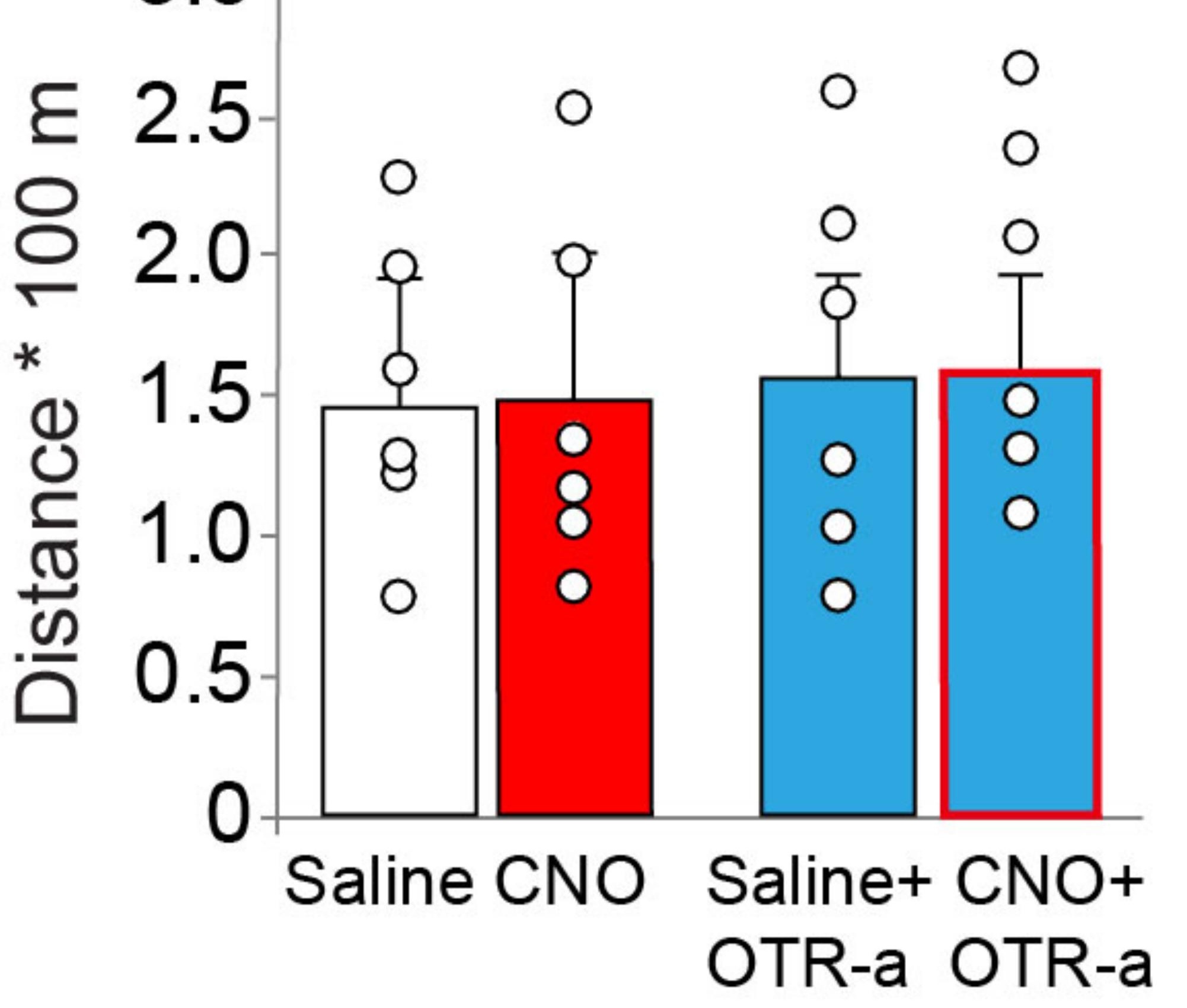

h

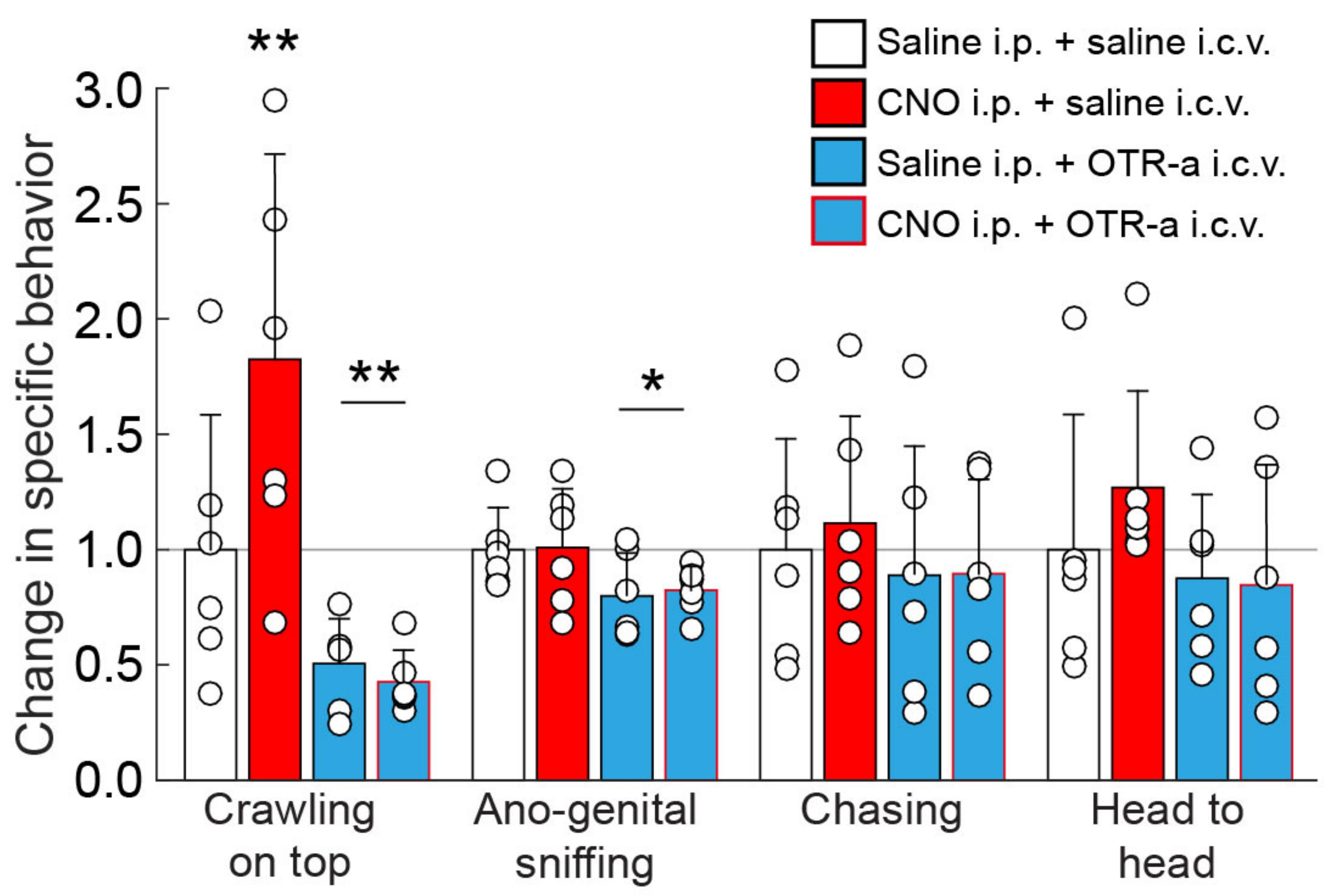

e

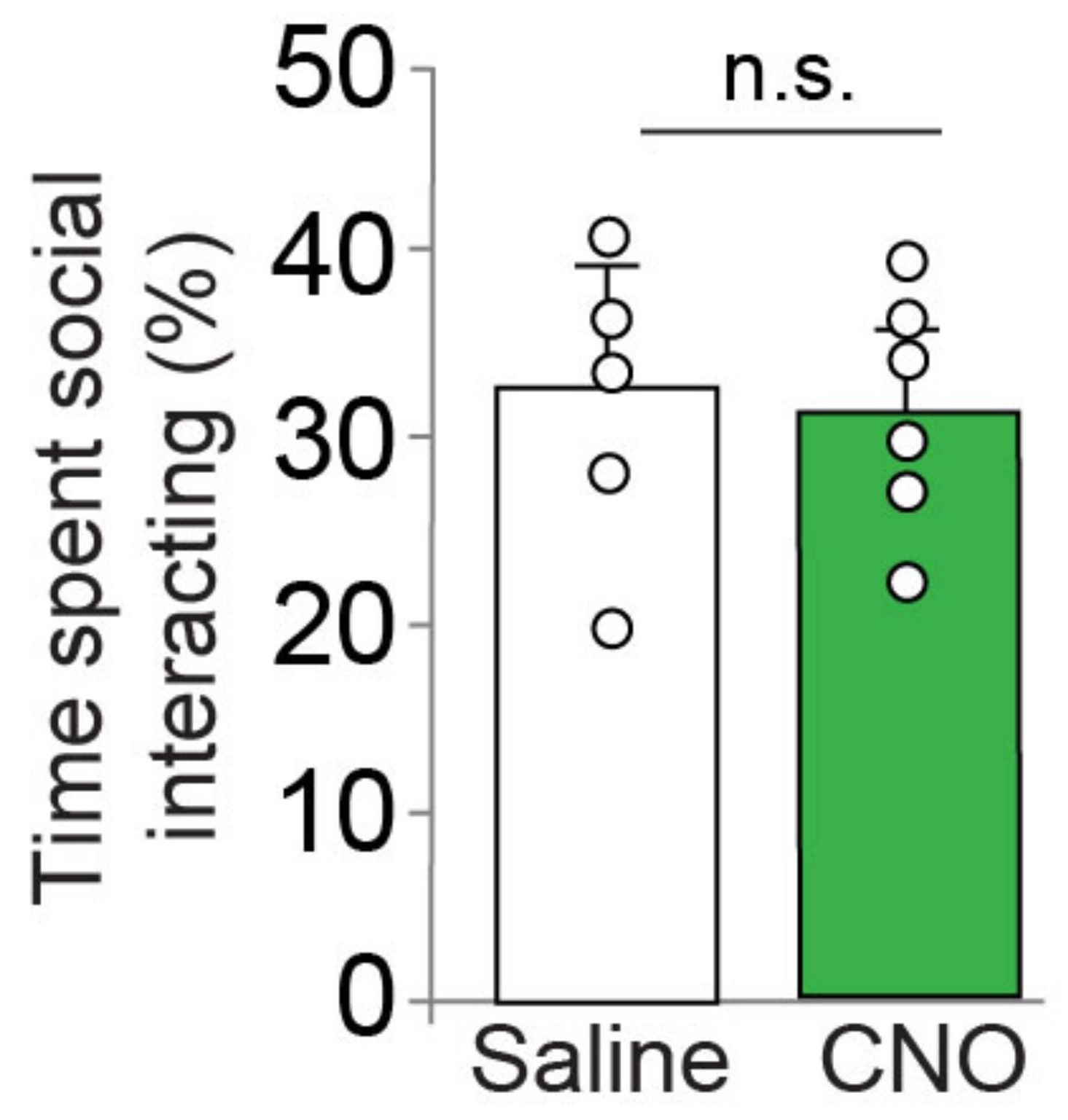

\section{Locomotor activity}

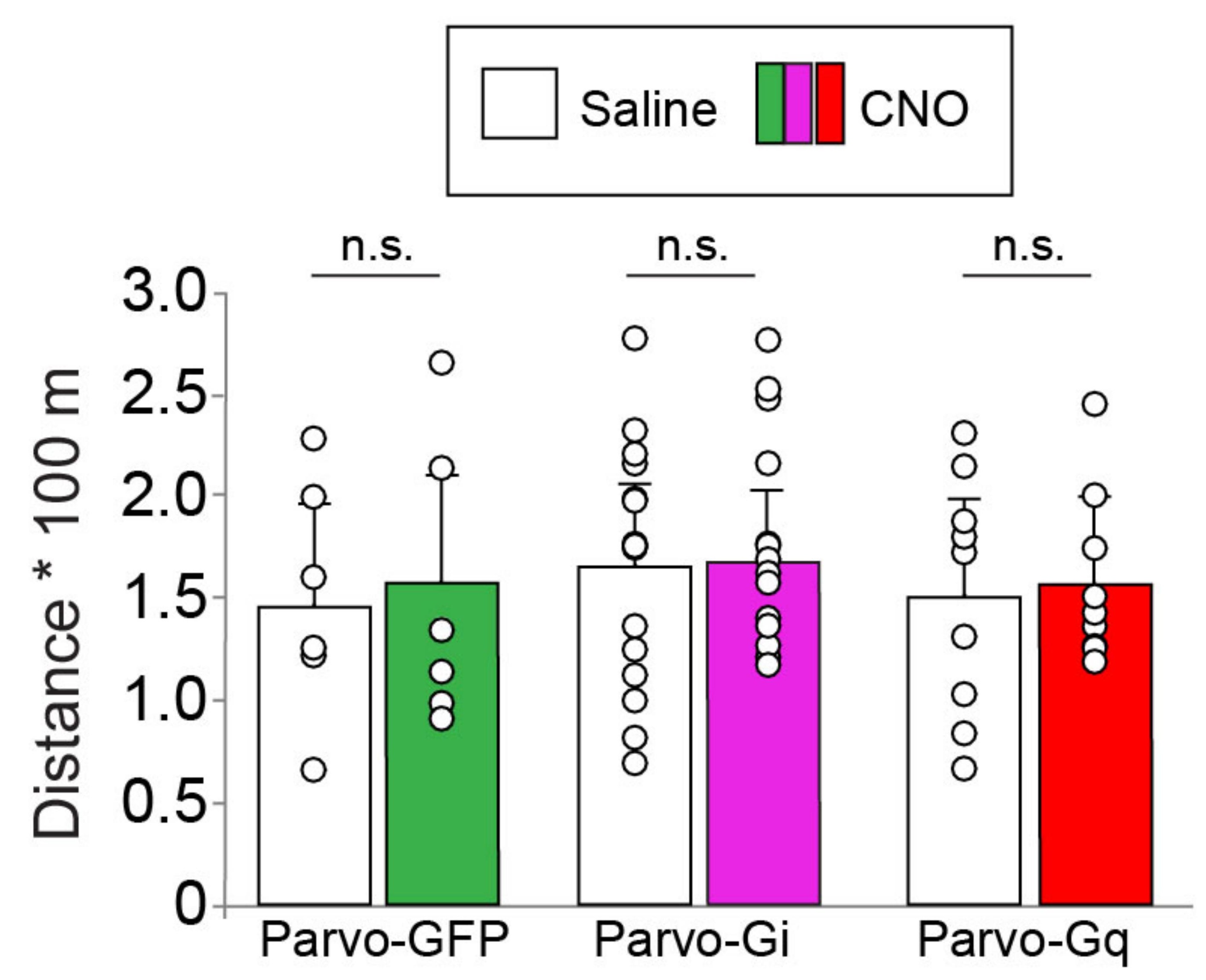

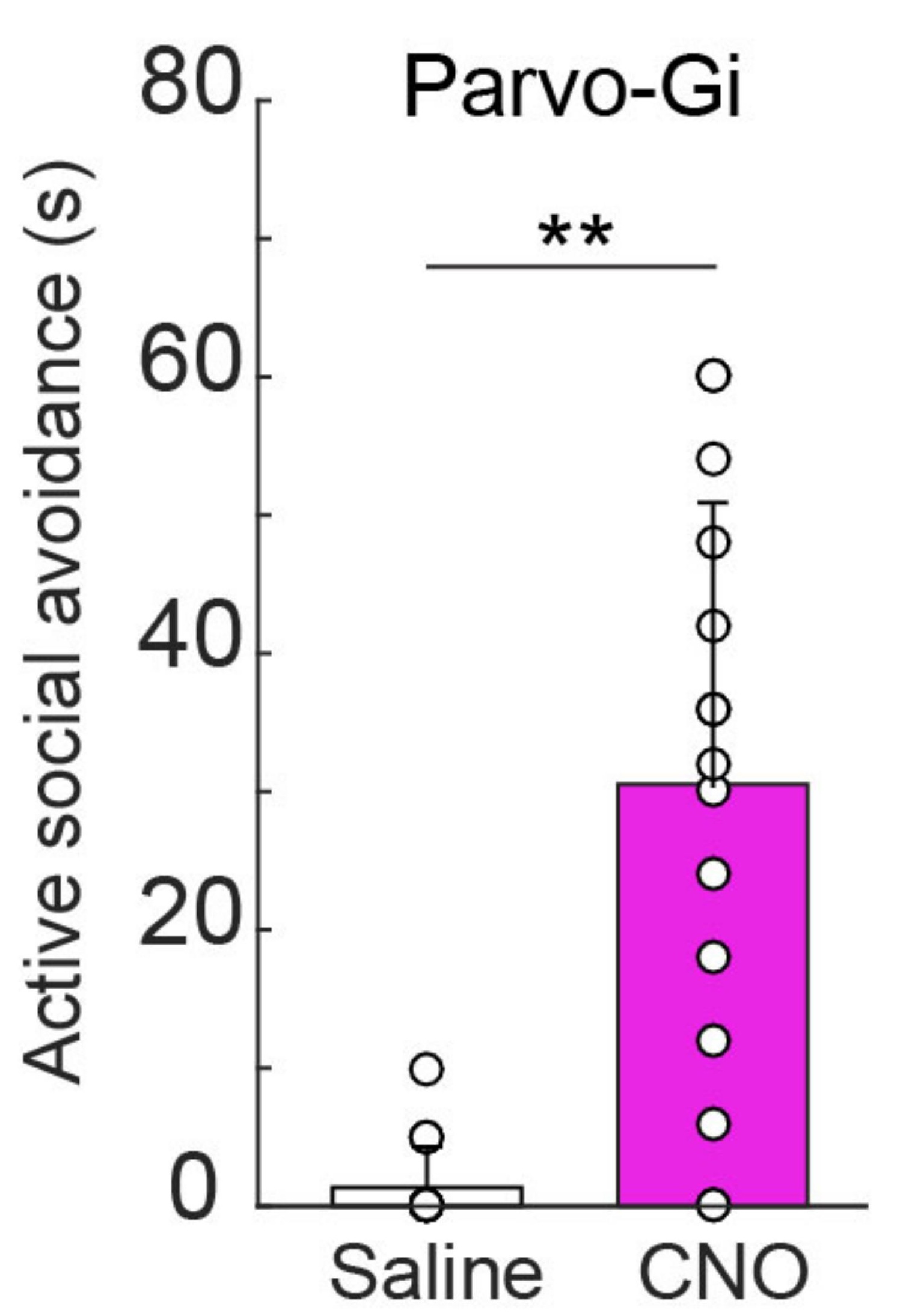

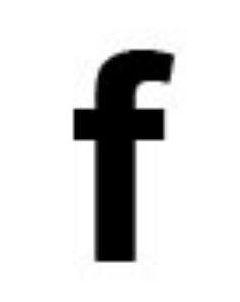

Locomotor activity

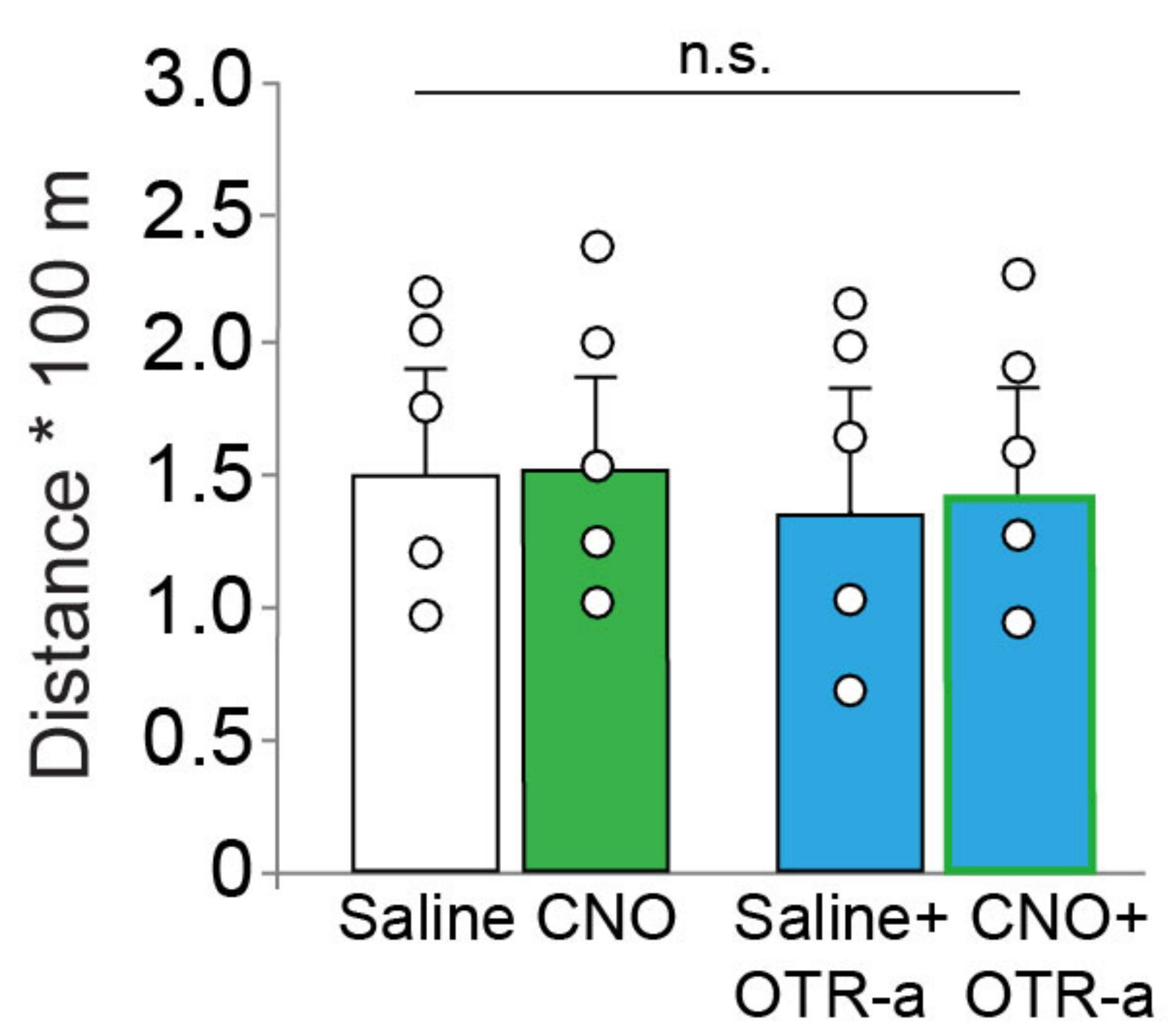

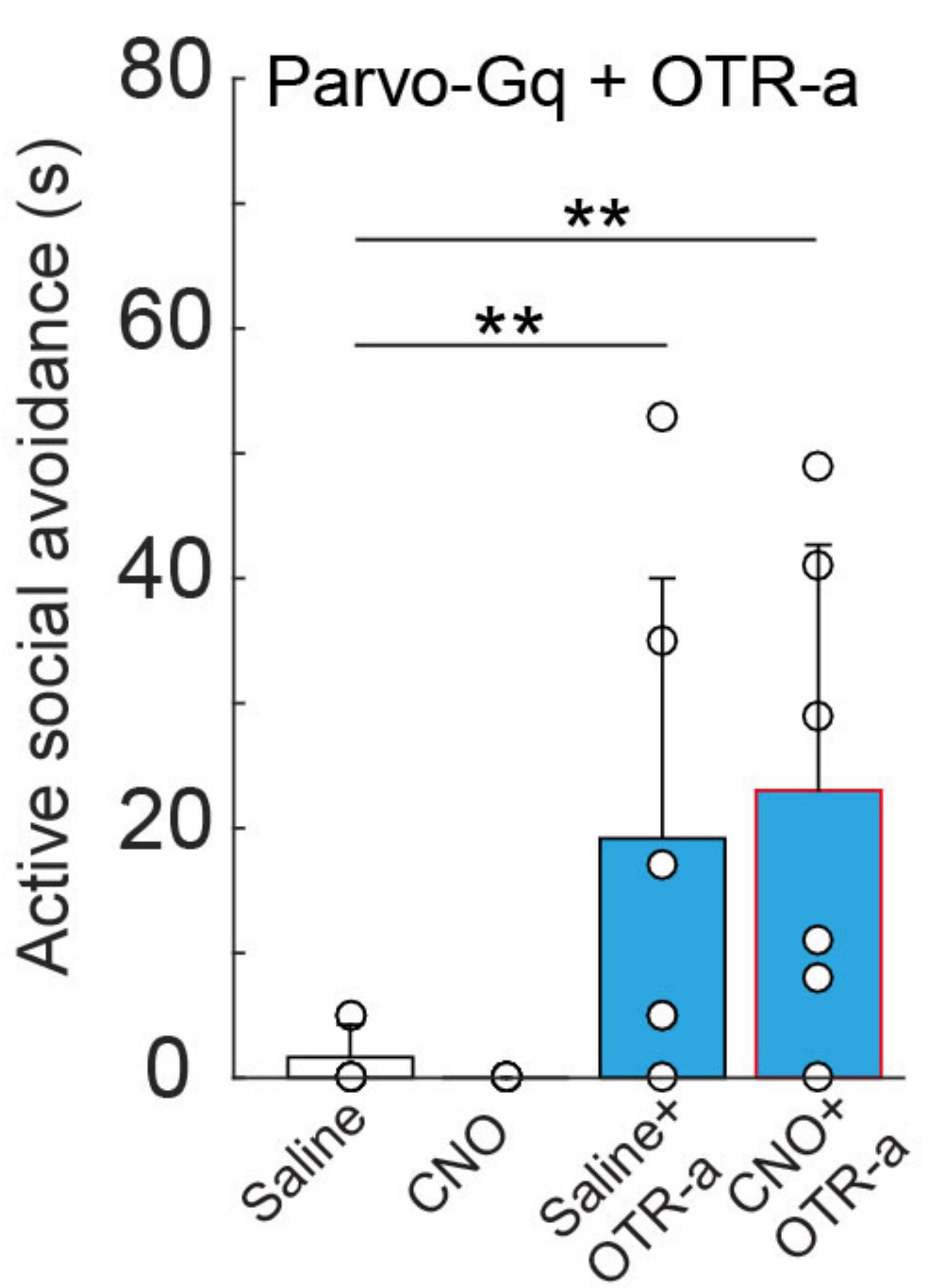
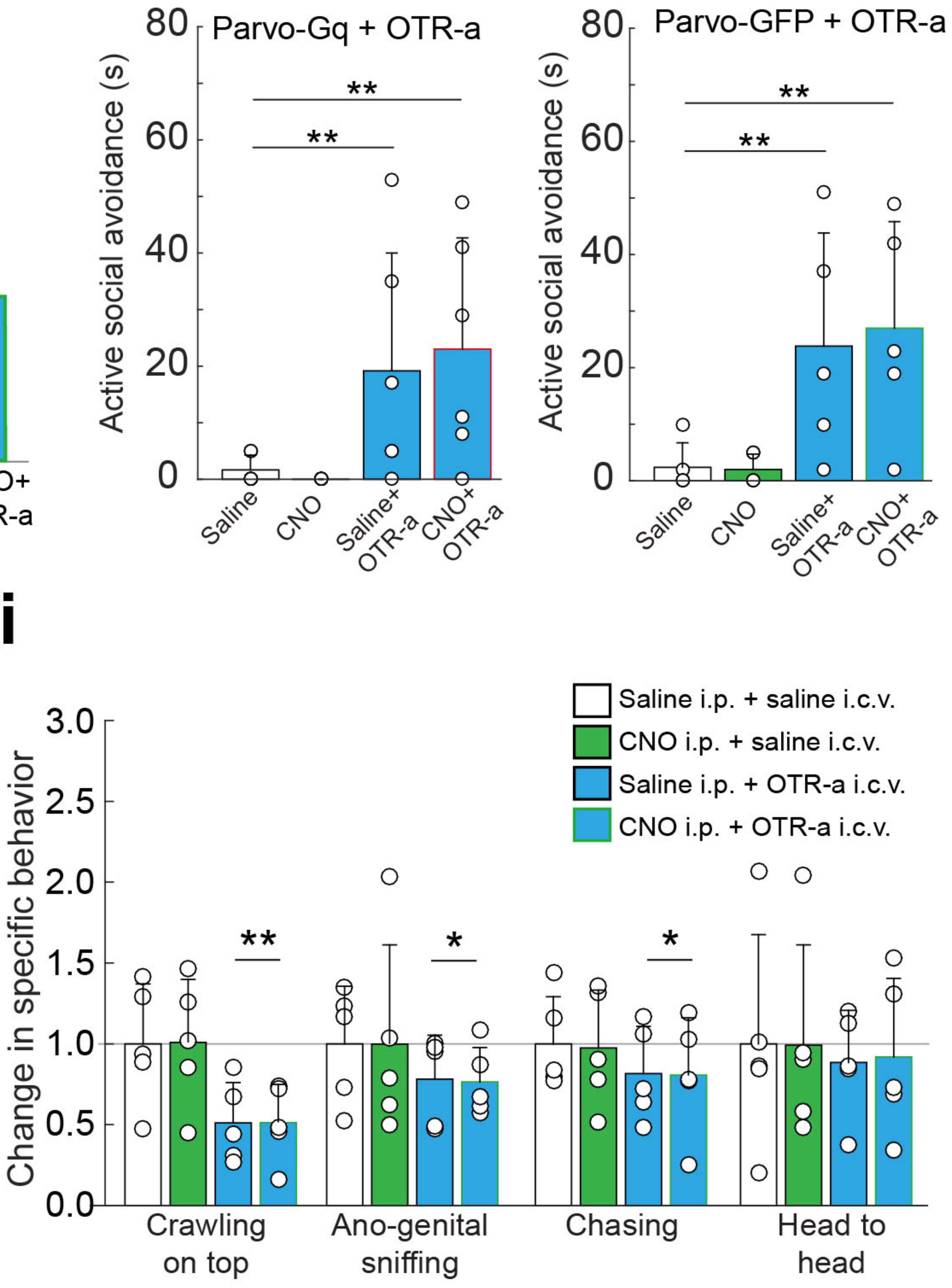


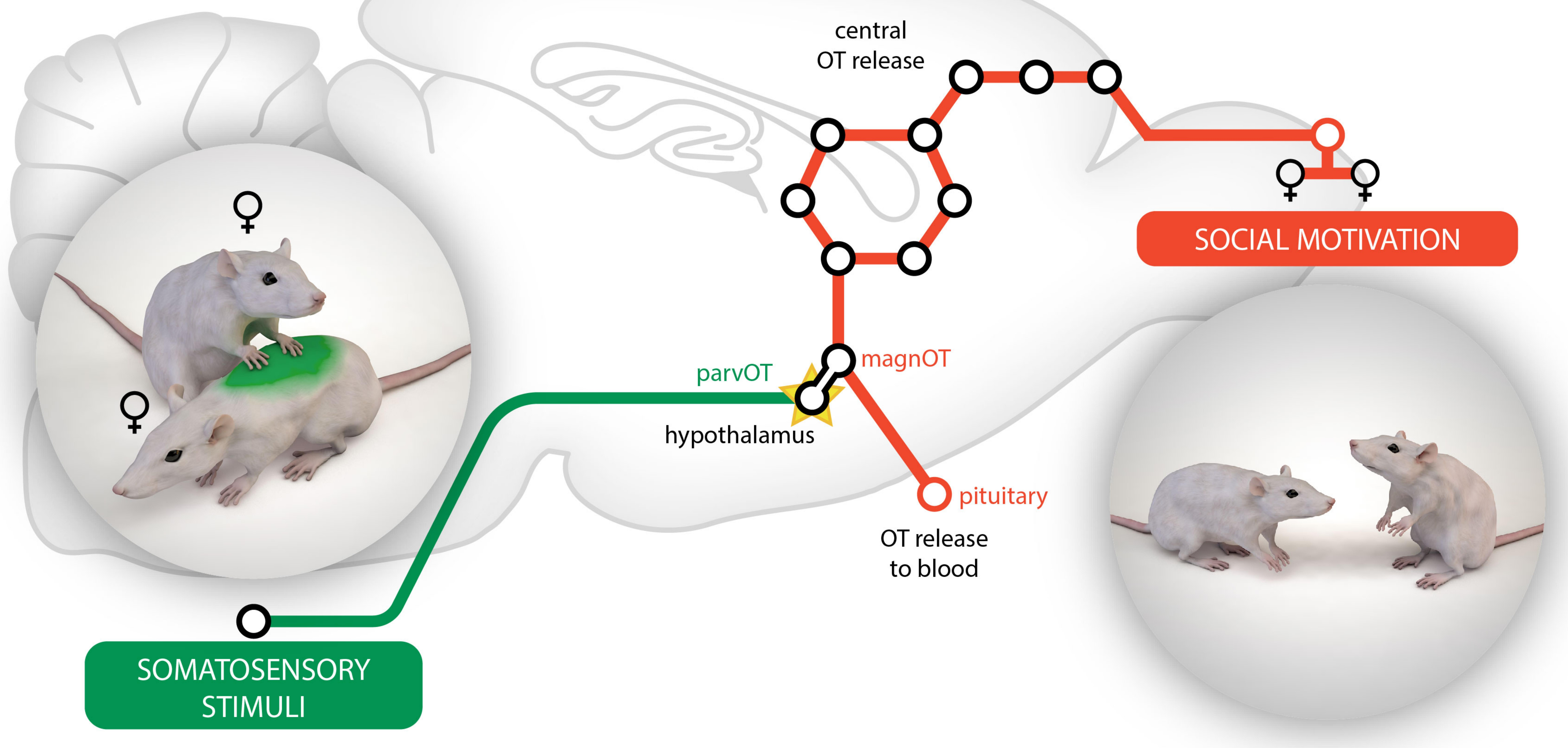

